\title{
Interconnection Impact Analysis of Solar Photovoltaic Systems with Distribution Networks
}

\author{
by \\ Michael Ikechi Emmanuel \\ A thesis \\ submitted to Victoria University of Wellington \\ in fulfilment of the \\ requirements for the degree of \\ Doctor of Philosophy \\ in Engineering.
}

Victoria University of Wellington 2018 



\begin{abstract}
As the solar PV technology continues to evolve as the most common distributed generation (DG) coupled with increasing interconnection requests, accurate modelling of the potential operational impacts of this game-changer is pivotal in order to maintain the reliability of the electric grid. The overall goal of this research is to conduct an interconnection impact analysis of solar PV systems at increasing penetration levels subject to the feeder constraints within the distribution network. This is carried out with a time series power flow analysis method to capture the time-varying nature of solar PV and load with their interactions with the distribution network device operations. Also, this thesis analyses multiple PV systems scenarios and a wide range of possible impacts to enable distribution system planners and operators understand and characterize grid operations with the integration of PV systems.

An evaluation of the operational and reliability performance of a gridconnected PV system based on IEC standards and industry guides is performed to detect design failures and avoid unnecessary delays to PV penetration. The performance analysis metrics in this research allow crosscomparison between PV systems operating under different climatic conditions. This thesis shows the significant impact of temperature on the overall performance of the PV system.

This research conducts an interconnection study for spatially distributed single-phase grid-tied PV systems with a five minute-resolution load and solar irradiance data on a typical distribution feeder. Also, this research compares the performance of generator models, PQ and $P|V|$, for connecting PV-DG with the distribution feeder with their respective computational costs for a converged power flow solution.
\end{abstract}


More so, a method capable of computing the incremental capacity additions, measuring risks and upgrade deferral provided by PV systems deployments is investigated in this research. This thesis proposes surrogate metrics, energy exceeding normal rating and unserved energy, for evaluating system reliability and capacity usage which can be a very useful visualization tool for utilities. Also, sensitivity analysis is performed for optimal location of the PV system on the distribution network. This is important because optimal integration of PV systems is often near-optimal for network capacity relief issues as well.

This thesis models the impact of centralized PV variability on the electric grid using the wavelet variability model (WVM) which considers the key factors that affect PV variability such as PV footprint, density and cloud movement over the entire PV plant. The upscaling advantage from a single module and point irradiance sensor to geographic smoothing over the entire PV footprint in WVM is used to simulate effects of a utilityinteractive PV system on the distribution feeder.

Further, the PV interconnection scenarios presented in this thesis have been modelled with different time scales ranging from seconds to hours in order to accurately capture and represent various impacts. The analysis and advancements presented in this thesis will help utilities and other stakeholders to develop realistic projections of PV systems impacts on the grid. Also, this research will assist in understanding and full characterization of PV integration with the grid to avoid unnecessary delays. 


\section{Publications From This Thesis}

A major part of this thesis has already been published in peer-reviewed journal articles, technical conferences and newsletters as listed below (the principal work was by the author of this thesis):

\section{Peer-reviewed Journals}

1. M. Emmanuel, R. Rayudu and I. Welch, "Impacts of power factor control schemes in time series power flow analysis for centralized PV plant using wavelet variability model," in IEEE Transactions on Industrial Informatics, vol.13, no.6, pp. 3185-3194, Dec. 2017.

2. M. Emmanuel, R. Rayudu, I. Welch, "Grid capacity released analysis and incremental addition computation for distribution system planning", In Elsevier Electric Power Systems Research, Volume 152, 2017, Pages 105-121, ISSN 0378-7796.

3. M. Emmanuel, D. Akinyele, R. Rayudu, "Techno-economic analysis of a $10 \mathrm{kWp}$ utility interactive photovoltaic system at Maungaraki school, Wellington, New Zealand", In Elsevier Energy, Volume 120, 2017, Pages 573-583, ISSN 0360-5442.

4. M. Emmanuel, R. Rayudu, "The impact of single-phase grid-connected distributed photovoltaic systems on the distribution network using P-Q and P-V models", In Elsevier International Journal of Electrical Power \& Energy Systems, Volume 91, 2017, Pages 20-33.

5. M. Emmanuel and R. Rayudu, Evolution of dispatchable photovoltaic system integration with the electric power network for smart grid applications: A review, Renewable and Sustainable Energy Reviews, 67 (10), 207-224, 2017. 
6. M. Emmanuel and R. Rayudu, Communication technologies for smart grid applications: A survey, Journal of Network and Computer Applications, 74 (10), 133-148, 2016.

\section{Peer-reviewed Conference Papers}

1. M. Emmanuel, R. Rayudu and I. Welch, "Impact of centralized photovoltaic systems on utility power factor profile using the wavelet variability model," 2017 IEEE Innovative Smart Grid Technologies Asia (ISGT-Asia), Auckland, 2017, pp. 1-6 (paper accepted for publication).

2. M. Emmanuel, R. Rayudu and I. Welch, "Grid incremental capacity evaluation with an optimally deployed photovoltaic system in distribution network," 2017 IEEE Power \& Energy Society General Meeting, Chicago, IL, USA, 2017, pp. 1-5.

3. M. Emmanuel and R. Rayudu, "Equipment loading and voltage unbalance in the distribution network with distributed PVs," 2016 IEEE Innovative Smart Grid Technologies - Asia (ISGT-Asia), Melbourne, VIC, 2016, pp. 9-14.

4. M. Emmanuel, R. Rayudu and D. Burmester, "Impact of large-scale integration of distributed photovoltaic with the distribution network," 2016 IEEE International Conference on Power System Technology (POWERCON), Wollongong, NSW, 2016, pp. 1-6.

\section{Peer-reviewed Newsletters}

1. M. Emmanuel, R. Rayudu and I. Welch, Techno-Economic Screening Analysis for Variable Renewable Energy Sources A ForwardLooking Alternative for a Seamless Baseload Generation Retirement, IEEE Smart Grid Newsletter, January 2018. 
2. M. Emmanuel, R. Rayudu and I. Welch, Distributed Energy Resources Operations in the Modern Grid, IEEE Smart Grid Newsletter, July 2017.

3. M. Emmanuel, R. Rayudu and I. Welch, Distributed Energy Resources Integration Standards for Smart Grid Applications, IEEE Smart Grid Newsletter, October 2016.

4. M. Emmanuel, R. Rayudu and W. Seah, Dispatchable Distributed Energy Resources for Seamless Integration into the Smart Grid, IEEE Smart Grid Newsletter, February 2016. 
vi

\section{Acknowledgements}

There is a spirit in man and the inspiration of the Almighty God gives him understanding. I am indeed indebted to Jesus Christ for His wisdom, grace and succour throughout this research adventure.

My profound gratitude goes to Victoria University of Wellington for providing the Victoria Doctoral Scholarship for me to be able to carry out my research in New Zealand. I would like to express my deep appreciation to my supervisors, Dr Ramesh Rayudu and Associate Prof Ian Welch, for their timely advice, support and guidance. My sincere thanks to Prof Winston Seah and Dr Bryan Ng for their support at some stage of my PhD program. I would also like to thank my research colleagues and the SPRES group for their constructive feedback and comments during our weekly presentations.

My deep appreciation also goes to my lovely wife (Folumi) and daughter (Shiloh) for their support, prayers and understanding throughout this research work. I would like to thank my other family members, in-laws and friends for their encouragement and prayers. Many thanks to my dear friend, Dr Daniel Akinyele, for his kind gestures, prayers and support. Special thanks to Dr and Dr Mrs Obed for their constant prayers and advice. Finally, I would want to thank Pastor Tale and Doreen with members of the Congregational Church in Wellington for their prayers and support. God bless you all in Jesus Name. 


\section{Contents}

1 Introduction 1

1.1 Problem statement . . . . . . . . . . . . . . 1

1.2 Motivation ..................... 2

1.3 Research Objectives . . . . . . . . . . . . . . 3

1.4 Major Contributions . . . . . . . . . . . . 5

1.5 Thesis Organisation . . . . . . . . . . . . . . . 8

2 Literature Review and Research Context 11

2.1 Overview of PV System Power Output Model . . . . . . . . 11

2.2 DERs Interconnection with EPS . . . . . . . . . . . . . 14

2.3 Overview of Grid-tied Inverter Technology . . . . . . . . . . 15

2.4 Related Work on Utility-interactive Inverter . . . . . . . . . . 19

2.5 Impacts of utility-interactive PV systems . . . . . . . . . . . . 21

2.6 Related work . . . . . . . . . . . . . . . . . 23

2.6.1 Voltage impacts ............... . 24

2.6.2 Impact on power losses . . . . . . . . . . . 25

2.6.3 Impact on voltage regulation devices . . . . . . . 26

2.7 Gaps in the related work . . . . . . . . . . . . . . . 27

2.8 Relevant PV-DG interconnection standards . . . . . . . . . 29

2.9 Research strategy . . . . . . . . . . . . . . . . . 31

2.10 Summary . . . . . . . . . . . . . . . . 34

2.11 Next chapter . . . . . . . . . . . . . . . . 34

vii 
3 Performance analysis of utility-interactive PV systems 37

3.1 Introduction . . . . . . . . . . . . . . . . 37

3.2 Description of the grid-tied solar PV system . . . . . . . . . . 40

3.3 System Performance Analysis . . . . . . . . . . . . . . . . . . 42

3.3.1 Reference yield . . . . . . . . . . . . . . 44

3.3.2 PV Array energy yield . . . . . . . . . . . . . . 44

3.3.3 Final system yield . . . . . . . . . . . . . 44

3.3.4 Performance ratio . . . . . . . . . . . . . 45

3.3 .5 Losses . . . . . . . . . . . . . . 45

3.3.6 Capacity factor . . . . . . . . . . . . 46

3.3.7 Inverter efficiency $\ldots \ldots \ldots . \ldots . \ldots 46$

3.3.8 System efficiency . . . . . . . . . . . . . . 46

3.3.9 Energy yield . . . . . . . . . . . . . . 46

3.4 Economic analysis . . . . . . . . . . . . . . . . . . 48

3.4.1 Net present value $(N P V) \ldots \ldots \ldots$. . . . . . . 48

3.4.2 Levelized cost of energy $($ LCOE) . . . . . . . . . . . 49

3.4.3 Simple payback period . . . . . . . . . . . . 49

3.5 Results and discussion . . . . . . . . . . . . . . . . . . 51

3.6 Comparison of PV systems performance . . . . . . . . . . 55

3.7 Economic analysis . . . . . . . . . . . . . . . . . 56

3.7.1 Actual economic analysis . . . . . . . . . . . . 60

3.8 Discussion . . . . . . . . . . . . . . . . 62

3.9 Summary ....................... 63

3.10 Next chapter . . . . . . . . . . . . . . . . 63

4 The impact of single-phase grid-connected distributed PV systems on the distribution network 65

4.1 Introduction . . . . . . . . . . . . . . 66

4.2 Load and solar PV modelling . . . . . . . . . . . . 68

4.2.1 Load modelling . . . . . . . . . . . . . . 68

4.2.2 Solar PV renewable resource modelling . . . . . . . . 69 
4.3 Distributed generation models . . . . . . . . . . . 71

4.3.1 Constant PQ node model . . . . . . . . . . . 72

$4.3 .2 \mathrm{P}|\mathrm{V}|$ bus model . . . . . . . . . . . . . 73

4.4 Problem formulation . . . . . . . . . . . . . . . 75

4.4.1 PV-DG impacts on the distribution network . . . . 75

4.4 .2 Voltage unbalance . . . . . . . . . . 79

4.5 Case study . . . . . . . . . . . . . . . . . . . 79

4.6 Simulation results and discussion . . . . . . . . . . . . . 81

4.6 .1 Solar generation . . . . . . . . . . . . . 82

4.6.2 The impact of distributed single-phase PV systems . 82

4.7 Chapter summary . . . . . . . . . . . . . . . . . . 88

4.8 Next chapter . . . . . . . . . . . . . . . . . . . . 89

5 Grid capacity released analysis and incremental addition computation for planning $\quad 91$

5.1 Introduction . . . . . . . . . . . . . . . . . 91

5.2 Energy quantities for incremental capacity measurement . . 94

5.3 System released capacity and loss analysis . . . . . . . . . . 95

5.4 Optimal sizing and placement of PV-DG . . . . . . . . . . . 97

5.4 .1 Optimal sizing . . . . . . . . . . . . 997

5.4 .2 Optimal placement . . . . . . . . . . . . . . 99

5.5 Annual load and irradiance characteristics . . . . . . . . . 101

5.5.1 Yearly load characteristics . . . . . . . . . . . . . . 101

5.5.2 Solar generation characteristic . . . . . . . . . . 101

5.6 Case study and simulation results . . . . . . . . . . . . . 103

5.6.1 IEEE 13-bus test feeder . . . . . . . . . . . . . . 103

5.6.2 IEEE 34-bus test feeder . . . . . . . . . . . . . . 104

5.7 Simulation results and discussion . . . . . . . . . . . . . . . 104

5.7.1 Basecase EEN . . . . . . . . . . . . . . . 105

5.7.2 EEN offset (capacity release) by PV-DG penetration . 106

5.7.3 Incremental capacity evaluation . . . . . . . . . 114 
5.7 .4 Energy Losses . . . . . . . . . . . . . . . . 115

5.7.5 UE offset by PV-DG penetration . . . . . . . . . 117

5.8 Chapter summary . . . . . . . . . . . . . . . . . . . . . . . 119

5.9 Next chapter . . . . . . . . . . . . . . . . . . . . . 120

6 Impacts of power factor control schemes in time series power flow analysis for centralized PV plants using wavelet variability model

6.1 Introduction . . . . . . . . . . . . . . . . . 122

6.2 Centralized solar PV system . . . . . . . . . . . . . . . . 125

6.3 Solar PV modeling . . . . . . . . . . . . . . . 126

6.4 Power factor control strategies . . . . . . . . . . . . 128

6.5 Case study . . . . . . . . . . . . . . . . . . 131

6.5.1 Feeder voltage impacts with PF control strategies . . 133

6.5.2 Impacts of variability on the voltage regulator/OLTC 137

6.5.3 Impacts on $\mathrm{kW}$ and $\mathrm{kVAR}$ profiles . . . . . . . . 140

6.6 Chapter summary . . . . . . . . . . . . . . . . . . . 143

6.7 Next chapter . . . . . . . . . . . . . . . . . . . . . 144

7 Conclusions and future research 145

7.1 Conclusions . . . . . . . . . . . . . . . . . . . 145

7.2 Future work . . . . . . . . . . . . . . 150 


\section{List of Figures}

2.1 The next generation electricity grid . . . . . . . . . . . . 14

2.2 DER interconnection with the EPS . . . . . . . . . . 15

2.3 Various configurations for utility-interactive inverter . . . . 16

2.4 PV inverter connection to grid . . . . . . . . . . . . . 17

3.1 Schematic block diagram of the PV system . . . . . . . . . 42

3.2 System performance analysis . . . . . . . . . . . . 43

3.3 Monthly averaged total in-plane irradiation and ambient temperature ..................... 52

3.4 Annual module temperature . . . . . . . . . . . . . . 52

3.5 Monthly capacity factors and performance ratios of the PV array .......................... 53

3.6 Monthly averaged daily final yield, PV array capture losses and system losses . . . . . . . . . . . . . . . . 54

3.7 Monthly system and inverter efficiencies . . . . . . . . . 54

3.8 WBPSMR monthly PV output energy . . . . . . . . . . 55

3.9 WBPSMR daily power generated . . . . . . . . . . . 56

3.10 Monthly energy consumption and peak demand . . . . . . . 59

3.11 Payback cash flow (simple payback period $=6.4$ years) $\ldots 60$

3.12 Annual electricity to/from the grid . . . . . . . . . . . . 60

3.13 School's energy usage and cost of energy for pre- and postinstallation of the PV array . . . . . . . . . . . . . . 61 
4.1 A typical daily solar irradiance with five-minute resolution during autumn in Wellington . . . . . . . . . . . . 71

4.2 Expected daily PV array output . . . . . . . . . . . . . 72

4.3 The modified IEEE-13 bus test network with single-phase PV 80

4.4 Normalized load profile of a school . . . . . . . . . . . . 81

4.5 Normalized daily irradiance with five-minute resolution . . 81

4.6 A typical active and reactive power injections at all singlephase load buses . . . . . . . . . . . . . . 82

4.7 Voltage profile along the feeder . . . . . . . . . . 83

4.8 Voltage profiles of buses on the heavily loaded black phase . 84

4.9 Voltage profiles of buses on the red phase . . . . . . . . 84

4.10 Voltage unbalance at 634,675 and 670 buses . . . . . . . . . 85

4.11 PV-DG impact on the equipment overloading . . . . . . . 86

4.12 Feeder power losses . . . . . . . . . . . . . . . . 87

5.1 Energy Exceeding Normal (EEN) and Unserved Energy (UE) concepts . . . . . . . . . . . . . . . 94

5.2 Power loss variation curves for all buses on the test feeder . 99

5.3 Annual energy demand profile . . . . . . . . . . . . . . 102

5.4 Annual irradiance profile . . . . . . . . . . . . . . 102

5.5 Modified IEEE 13-bus test feeder. The modification refers to the presence of PV-DG . . . . . . . . . . . . . 103

5.6 Loss and voltage sensitivities . . . . . . . . . . . . . . 103

5.7 Modified IEEE 34-bus test feeder. The modification refers to the presence of PV-DG . . . . . . . . . . . . . . . . 104

5.8 Loss and voltage sensitivities . . . . . . . . . . . . . 105

5.9 Basecase EEN at peak load for both systems . . . . . . . . . 106

5.10 A 5-year period growth of EEN for IEEE 13-bus . . . . . . . 107

5.11 A 5-year period growth of EEN for IEEE 34-bus . . . . . . 108

5.12 IEEE 13-bus capacity release with 15\% PV-DG . . . . . . . 109

5.13 IEEE 34-bus capacity release with 15\% PV-DG . . . . . . . 110

5.14 IEEE 13-bus capacity release with optimal size PV-DG . . 110 
5.15 IEEE 34-bus capacity release with optimal size PV-DG . . . 111

5.16 IEEE 13-bus EEN reduction with load growth for all PV-DG cases . . . . . . . . . . . . . . . . . 112

5.17 IEEE 34-bus EEN reduction with load growth for all PV-DG cases .......................... 113

5.18 Basecase annual energy losses at peak load for both systems 113

5.19 IEEE 13-bus incremental capacity computation based on EEN114

5.20 IEEE 34-bus incremental capacity computation based on EEN115

5.21 Basecase annual energy losses at peak load for both systems 116

5.22 Comparison of losses with load growth for all cases . . . . . 117

5.23 IEEE 13-bus unserved energy for all PV-DG cases . . . . . . 117

5.24 IEEE 34-bus unserved energy for all PV-DG cases . . . . . . 118

6.1 Distribution-interactive utility-scale PV system . . . . . . 125

6.2 WVM smoothed irradiance for the input irradiance . . . . . 127

6.3 Variability reduction at different timescales . . . . . . . . . 128

6.4 A typical power factor schedule . . . . . . . . . . . . . . . . . 129

6.5 A typical power factor as a function of PV output . . . . . . 130

6.6 Active power produced at various $P_{L}$ modeled using WVM 130

6.7 Reactive power produced at various $P_{L}$ modeled using WVM130

6.8 Annual normalized demand profile for various customers . 131

6.9 Modified IEEE 34-bus test feeder. The modification refers to the presence of PV-DG . . . . . . . . . . . . . . . . 132

6.10 Satellite image of IEEE-34 bus topology in Phoenix, Arizona (black line is for three phase lines used in connecting centralized PV systems) . . . . . . . . . . . . . . . 133

6.11 Maximum voltage with PV close to feeder end . . . . . . . . 134

6.12 Maximum voltage with PV close to feeder source . . . . . . . 134

6.13 Minimum voltage with PV close to feeder source . . . . . . . 135

6.14 Minimum voltage with PV close to feeder end . . . . . . . 135

6.15 Feeder voltage profile with no PV system . . . . . . . . . 136

6.16 Feeder voltage profile with PV at unity PF close to the FS . . 136 
6.17 Feeder voltage profile with PV at unity PF close to the FE . . 136

6.18 Feeder voltage profile with PF schedule PV close to the FS . 137

6.19 R1 and R2 LTC operations in the sample week (Basecase) . . 138

6.20 R1 and R2 LTC operations in the sample week with PV plant at FE . . . . . . . . . . . . . . . . . . . . . 138

6.21 Total tap operations in the sample week for the basecase and PV deployed at FS with various PF control strategy . . . . . 139

6.22 Total tap operations in the sample week for the basecase and PV deployed at FE with various PF control strategy . . . . . 139

6.23 Basecase $\mathrm{kW}$ and $\mathrm{kVAR}$ power flows on the feeder lines . . . 141

$6.24 \mathrm{~kW}$ power flows offset with PV systems . . . . . . . . . . . 141

6.25 Comparison of kVar power flows with different PF strategies 143 


\section{List of Tables}

3.1 Electrical properties of the inverters. . . . . . . . . . . . . 41

3.2 PV module specifications . . . . . . . . . . . . . . . . 43

3.3 Grid-connected PV system performance evaluation in various locations . . . . . . . . . . . . . . . 57

3.4 Inputs and assumptions used in the analysis . . . . . . 58

3.5 Cash flow . . . . . . . . . . . . . . . . . . . . 59

3.6 Economic measures evaluation of the $10 \mathrm{kWp}$ system . . . . 59

4.1 Load types and corresponding voltage exponents . . . . . 69

4.2 Single-phase load buses of IEEE-13 test network . . . . . . . 81 


\title{
Nomenclature
}

\author{
API Application Programming Interface \\ CAIDI Customer Average Interruption Duration Index \\ CAIFI Customer Average Interruption Frequency Index \\ $D G \quad$ Distributed Generation \\ DTIM Demand Time Matching \\ ECP Equivalent Conventional Power \\ EEN Energy Exceeding Normal \\ EFC Equivalent Firm Power \\ ELCC Effective Load Carrying Capacity \\ EPS Electric Power System \\ EUE $\quad$ Expected Unserved Energy \\ FE $\quad$ Feeder End \\ FS $\quad$ Feeder Source \\ GIS Geographic Information System \\ GridPV Grid Integrated Distributed PV \\ xvii
}


IEAPVPS International Energy Agency Photovoltaic Power System

LCOE Levelized Cost of Energy

LDC Load Duration Capacity

LF $\quad$ Load Factor

LSI Loss Sensitivity Index

NPV Net Present Value

OLTC On-load Tap Changer

OpenDSS Open Distribution System Simulator

$P_{L} \quad$ Penetration level

PCC Point of Common Coupling

PDE Power Delivery Element

PF $\quad$ Power Factor

PLCC Peak Load Carrying Capacity

PR Performance Ratio

pu Per unit

PV Photovoltaic

$R M S \quad$ Root Mean Square

$U E \quad$ Unserved Energy

$V R \quad$ Variability Reduction

VREG Voltage Regulator

VSI Voltage Sensitivity Index

WVM Wavelet Variability Model 


\section{Chapter 1}

\section{Introduction}

\subsection{Problem statement}

The solar photovoltaic (PV) system has become an important resource for electric power generation in the 21st century due to the global quest for sustainable and clean energy [1], [2]. The ongoing incentivization of PV systems uptake have led to a high penetration of this game-changing technology in the conventional electric power systems (EPS). The PV technology is currently being referred to as the most common distributed generation (DG) integrated with the EPS [3]. High PV penetration with diverse scenarios on the same distribution feeder now requires more detailed modelling and analysis to avoid unnecessary delays in its integration with the EPS.

Therefore, with the increased PV interconnection requests there is a need for accurate modelling of the grid-connected PV system aggregate variability to enable utilities and authorities having jurisdiction (AHJ) over the grid to fully characterize its impacts and provide mitigation alternatives [4], [5]. The PV-DG grid integration issues include change in voltage regulation device operations, feeder power factor profile and voltage fluctuations, and increased network capacity. These impacts, local or systemwide have to be understood in order to provide suitable smart grid mea- 
sures and for proper characterization of the grid operation [4].

\subsection{Motivation}

The recent reduction in the cost of PV modules with higher efficiency has led to a very significant growth of PV systems deployment at an average annual rate of about $60 \%$ [6], [7]. This increasing integration of PV systems in the grid with a global installed capacity at $227 \mathrm{GW}$ and about $50 \mathrm{GW}$ of additional capacity in 2015, calls for a detailed interconnection study with high penetration scenarios [8]. Utilities show a lot of scepticism with PV interconnection with the grid because of the intermittent nature of the solar resource, estimation of its value and associated grid integration issues [9]. This fear expressed by the management of utilities prevents the inclusion of the PV-DG value in the distribution planning and therefore, constitute a major set-back in its adoption and integration with the EPS. Utilities need a more accurate modelling and representation of multiple grid-connected PV scenarios to assess planning and operating effects [10]. Also, for such grid-level application, it is now pertinent to provide AHJ and regulators with relevant guidance to recognize the true value PV-DG brings to the distribution network reliable operations [9].

Grid-connected PV systems have the tendency to cause emergent behaviours in the traditional electricity infrastructure due to its inherent intermittency, uncertainty and location-specificity [6], [11]. Some of the related grid integration issues include voltage or power quality problems, increase in operation of voltage regulating devices and equipment overloading. These effects can be localized (e.g., voltage and power quality problems) or system-wide (network balancing and potential interaction with other technologies types such as energy storage) [4]. These impacts are clear indications that the significant penetration of the PV technology in the distribution network calls for its inclusion in the active distribution system planning in order to maintain the EPS reliability and resiliency. 
Further, studying these impacts requires modelling with different time scales ranging from seconds to hours in order to accurately capture and represent various impacts [12]. For instance, the effect of PV output variability on the voltage regulating devices such as the on-load tap changer requires a high frequency irradiance data in seconds time scale [12], [13]. However, hourly and sub-hourly operational impact analysis would be useful to provide a wide overview of the PV generation impact on system performance, for example, plant energy production and additional load serving capacity provided by PV systems [12], [14].

Moreover, it is still not clear to utilities as to how renewable DG are to be considered in distribution planning process and the evaluation of their incremental capacity contributions remains a key issue. For effective planning, the inclusion of renewable DG such as PV systems is very important because of their additional load serving capacity provided to the distribution network, which invariable enhances system reliability [15], [16], [17].

In addition, in order to capture and analyse a wide range of possible impacts, it is important to investigate a diversity of PV scenarios on the distribution network. This will enable distribution system planners and operators to understand and characterize grid operation with the integration of the PV system [13], [18].

The need to develop realistic projections with accurate modelling of utility-interactive PV systems has become imperative. Therefore, to enable a graceful uptake of the PV technology into the evolving active distribution system, accurate modelling is pivotal for true impact assessment and the provision of smart grid mitigation alternatives.

\subsection{Research Objectives}

The overall goal of this thesis is to conduct an interconnection impact analysis of solar PV systems at increasing penetration levels subject to the feeder constraints with the distribution network. This is carried out in 
a time series power flow analysis with varying load demand profiles. This study uses different solar irradiance resolutions and considers diverse PV scenarios in order capture the true impacts of PV integration and characterize appropriately post-installation grid operation.

In order to achieve this goal, a set of research objectives have been established as follows:

1. Evaluate the operational and reliability performance of the utilityinteractive PV system based on the IEC standards, IEA photovoltaic power systems program (PVPS) and industry guides such as the clean energy council. The performance analysis of a grid-connected PV system in a primary school will be carried out as a case study.

2. As an extension to the first objective, this study develops a model that integrates a typical distribution network and spatially distributed single-phase PV systems using a typical school load profile. This is required to understand and evaluate the impacts of single-phase units on the feeder. Also, this thesis aims to compare the performance of different DG interconnecting models to minimize the adverse impacts while maximizing the value of connected PV-DG.

3. Compute PV-DG incremental capacity additions on heavily and lightly loaded distribution feeders. This evaluation is based on the capacity constraints of the power delivery elements (PDE) rather than the usual computationally intensive probabilistic index referred to as the effective load carrying capacity. This study performs risk and upgrade deferral measurements for both networks using PDE ratings. There is a strong correlation between capacity and power losses in a distribution network. This implies that optimally deployed PV-DG (in terms of location and size) to minimize power losses is often nearoptimal for system capacity release. Therefore, a set of sub-objectives are identified as follows: 
- Sensitivity analysis for optimal placement of PV-DG in the distribution test feeders.

- Develop a model to generate a power loss variation pattern to obtain optimal sizes at different buses in the network.

4. Model the impact of variability and locational value of centralized PV plants using the wavelet variability model (WVM). The variability observed from a single point irradiance sensor cannot provide the exact representation of variability across the entire plant. This model performs geographic smoothing with a high frequency irradiance data over the PV footprint for accurate modelling of PV plant output variability. In addition, this study models various power factor control schemes and their impacts for the integrated PV-DG at different penetration levels. Multiple PV scenarios with different varying load demand profiles in high resolution time series simulation will be investigated. This will help in capturing the various impacts of PV integration and accurately characterize grid operation.

\subsection{Major Contributions}

The contributions of this thesis are as follows:

1. This thesis shows how to evaluate the operational and reliability performance of a grid-connected PV system based on the IEC standards (61836 and 61724), IEA PVPS and industry guides. The performance analysis metrics in thesis allow cross-comparison between PV systems operating under different climatic conditions and detection of operational faults. Results showed the significant impact of temperature on the overall performance of the PV system. Periods with low temperatures experienced higher performance ratio values, reduced array captured and system losses, while the reverse is true for 
higher temperature seasons. These results have been peer-reviewed and published in [19].

2. There are two types of DG models for active distribution system power flow, they are: constant PQ and $P|V|$ models. This thesis compares the performance of these models in terms of local impacts such as bus voltage profiles and unbalance, and system-wide impacts which include feeder equipment loading, power losses and computational cost for a converged power flow solution. This thesis proposes the use of $P|V|$ over PQ model for integrating PV-DG with distribution network because it minimizes both local and systemwide impacts with reduced computational cost. These results have been peer-reviewed and published in [20].

3. The conventional reliability indices (CAIDI, SAIFI, etc.) use average values, which makes it difficult to notice a change if small number of consumers experience enhancement in reliability as a result of DG integration. This thesis proposes surrogate metrics, energy exceeding normal rating and unserved energy, for evaluating system reliability which can be used as a visualization tool for distribution network planners and operators. This thesis shows how these metrics can be used to compute PV-DG incremental capacity additions and measurement of the period of upgrade deferral of the distribution system. Also, since a single year analysis may not be sufficient to characterize the dynamic distribution network due to the inevitable load growth, this study presents multi-year analysis considering a yearly demand growth on both lightly and heavily loaded networks. The analysis shown in this thesis is very useful in situations where the rate of demand increase is uncertain and the expansion of the network is very difficult and expensive. These results have been peerreviewed and published in [21],[22].

4. This thesis shows how to model impacts of variability and locational 
value of centralized PV plants in a distribution system with a high resolution solar irradiance data (1 s) and geographic smoothing using the WVM. Here, a duty cycle dispatch simulation is used to investigate the true impacts of solar variability at various penetration levels, which is an important step in incorporating such a variable resource in the EPS operations. Further, the WVM is used to estimate the output of a centralized PV plants operating with three power factor control strategies (power factor function, power factor schedule and fixed power factor) at various penetration levels. Analysis of scenarios, namely, PV-DG close to the feeder source and end for centralized PV deployments on the IEEE-34 bus feeder are presented. Impacts on the feeder active and reactive power profiles, voltage regulator tap changing operations, maximum and minimum feeder voltages are investigated. Also, two types of customers (i.e., commercial and residential) with different time-varying load patterns for an entire year (8760 hours) are incorporated in the distribution test feeder. Also, this research proposes the use of advanced inverter functionalities such as power factor function and schedule control strategies over the fixed power factor technique. This is because the former provides the flexibility required by utilities to adjust the PV output without assuming a particular amount of plant output each day. These results have been peer-reviewed and published in [23].

Overall, in this thesis, an intensive use of computer simulations have been used to conduct steady state local and system-wide analysis for varying sizes of PV-DG. This thesis provides a general understanding of the evolving active distribution system with guidelines and recommendations for its effective and reliable operations. 


\subsection{Thesis Organisation}

The remainder of this thesis is organized as follows:

Chapter 2 presents an overview of distributed energy resource (DER) and its interconnection with the distribution system. Emphasis is placed on the integration of PV technology as a typical example of DER. An overview of PV system performance model is presented with the various steps involved. In addition, this chapter presents a review of the operational local and system-wide impacts of integrating PV-DG on the distribution network. The last part of this chapter discuses DG interconnection requirements and standards. The literature review in this chapter has led to the publication of [24].

In Chapter 3, the evaluation of the operational and reliability performance of the utility-interactive PV system based on the IEC standards, IEA photovoltaic power systems program (PVPS) and industry guides such as the clean energy council is presented. This methodology is applied for the performance analysis of the $10 \mathrm{kWp}$ grid-connected PV system at Maungaraki school, Wellington, New Zealand as a case study. The research output presented in this chapter has resulted in the publication of [19].

In Chapter 4, a timeseries method for modelling grid-connected PV systems is presented. The DG interconnecting models, constant PQ and $P|V|$ models, are discussed. Active distribution power flow analysis and grid-tied PV modelling are presented. Further, the impact of single-phase utility-interactive PV systems distributed on the IEEE 13-bus test feeder using the different DG models is investigated. The results presented in this chapter have resulted in the publication of [20].

In Chapter 5, sensitivity analysis and power variation curves for optimal location and sizes of PV-DG on lightly and heavily loaded networks are presented. The computation of integrated PV-DG incremental capacity addition for serving network peak loads in a multi-year analysis is pre- 
sented. This is done based on two energy metrics, energy exceeding normal and unserved energy, which are dependent on the PDE normal and emergency ratings. The research output presented in this chapter have resulted in the publication of [21], [22].

In Chapter 6, the WVM for modelling centralized PV plants is discussed. This chapter shows how to model the impacts of variability and locational value of centralized PV plants in a distribution system with a high resolution solar irradiance data ( $1 \mathrm{~s})$ and geographic smoothing using the WVM. Further, the WVM is applied in estimating the output of a centralized PV plants operating with three power factor control schemes (power factor function, power factor schedule and fixed power factor) at various penetration levels. Analysis of three scenarios, namely, PV-DG close to the feeder source, midpoint and end for centralized PV deployment on the IEEE-34 bus feeder are presented. The results presented in this chapter have been published in [23].

Chapter 7 presents a summary of the key findings and conclusions of the research in this thesis. Also, this chapter includes the contributions and future research directions. 


\section{Chapter 2}

\section{Literature Review and Research Context}

This chapter presents an overview of PV system performance model with the various steps involved. Also, a review of distributed energy resources (DERs) and their interconnection requirements with the distribution system is presented. This study focuses on the integration of PV technology as a typical example of DER. In addition, this chapter presents a review of the operational local and system-wide impacts of integrating PV-DG on the distribution network. The last part of this chapter discuses DG interconnection requirements and standards.

A major part of this literature review has been peer-reviewed and published in [24].

\subsection{Overview of PV System Power Output Model}

In order to maintain the grid reliability, utilities expect a PV power plant to operate continuously at its maximum designed efficiency [25]. Apart from the effects of aging and variability in power generation caused by climatic conditions such as solar irradiance and temperature, distribution network planners anticipate PV systems to have maximum yield under 
optimal environmental conditions [25]. For grid integration studies, the PV power output model can be useful in the following ways [25], [26], [27]:

- Sizing of the PV system for a particular application that is based on anticipated power generation on an hourly, monthly, or yearly basis.

- Evaluation of PV unit power rating by converting measured parameters to performance at standard test conditions.

- Monitoring of the actual array performance against expected or predicted performance over the lifetime of the PV system.

- Early detection of electrical failures such as cable disconnection.

- Evaluation of PV units efficiency degradation caused by factors such as dust collection on panels and cable dissipation as a result of contact oxidation.

The factors to be considered in the PV plant power output model include the following [26], [28], [29]:

1. Irradiance and weather: The solar irradiance data is usually obtained from a meteorological model or database which provides hourlyaverage irradiance values and weather data such as the temperature and wind speed. Irradiance represents an instantaneous measurement of solar power received per unit area.

2. Incidence irradiance : In predicting PV plant output, it is very important to determine the irradiance incident on the plane of the array (POA) which depends on time. The irradiance is translated to the POA taking into consideration the impacts of array orientation and tracking, diffuse and beam irradiance, and ground surface reflectivity. 
3. Soiling, shading and reflection losses: These factors can significantly affect plant output by causing less amount of irradiance to reach the PV array.

4. Cell temperature: This factors scales the PV system output and acts as the highest contributor to non-linearity when converting solar irradiance to power. An increase in cell temperature reduces its efficiency, and rapid changes in cell temperature as a result of increased wind cooling increases the tendency of PV variability. Also, cell temperature is a function of many factors such as array mounting, ambient air temperature, module materials, wind speed and direction.

5. Module I-V output and DC mismatch losses: The I-V curve for PV modules depends on the irradiance, temperature and cell material. For a string of PV modules connected in series, DC mismatch occurs as a result of small variations in the maximum power point (MPP) of the modules. This change in the MPP can affect the PV system output variability such that a non-linearity occurs between irradiance and power.

6. DC to AC conversion losses: The conversion efficiency from DC output power from the PV array to grid compatible AC power depends on the inverter specifications such as efficiency and operating output power factor. Inverters are usually oversized (e.g., by $10 \%$ ) to avoid reaching their saturation points and limiting the PV unit AC output. IEEE 1547 and IEC 62116 recommends grid-connected inverters to rapidly disconnect from the network within 2 seconds during a loss of grid condition and should not attempt to reconnect until after 5 minutes.

7. AC losses: These can be significant especially for utility scale PV plants, which exist between the inverter AC side and the point of common coupling with the grid. 


\subsection{DERs Interconnection with EPS}

DERs represent a group of energy resources which provide myriad of possibilities such as electric energy generation, conversion, storage and interconnection to the EPS [30], [31]. They include photovoltaic (PV) arrays, gas-fired turbines, mircroturbines, wind turbines, fuel cells, reciprocating engines, conventional diesel and natural gas, and energy storage technologies. These are broadly classified into rotating prime mover technologies (such as wind turbines, microturbines) and non-rotating prime mover technologies such as PV and fuel cells [30].

The traditional assumption of the substation being the only source of power and short-circuit capacity is no longer valid as a result of DERs integration with the distribution system as shown in Figure 2.1 [32]. The

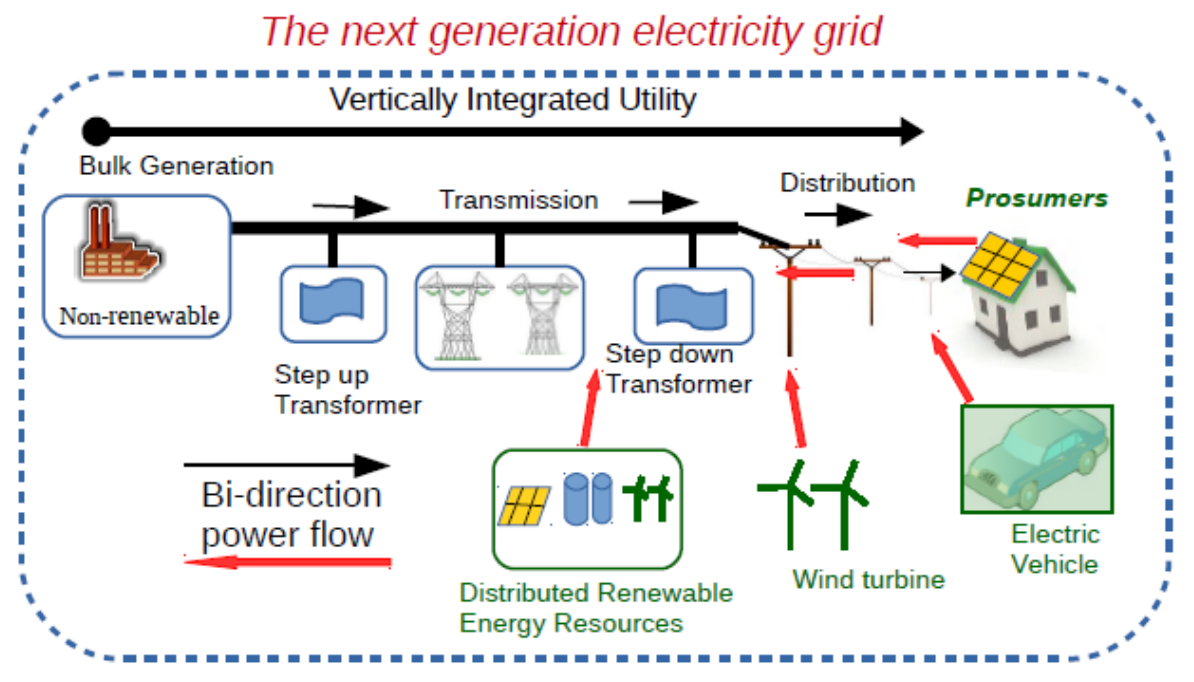

Figure 2.1: The next generation electricity grid

vertically integrated utility with all domains of generation, transmission and distribution having a sole owner is now divided amongst different stakeholders. The power generation market is undergoing deregulation with prosumers being able to feed back to grid excess power generated [33]. 
DER connects with the area EPS at the point of common coupling (PCC) (as shown in Figure 2.2) which depends on the interconnection requirements specified by the utility and authorities having jurisdiction (AHJs) over the grid [30], [33]. The total capacity of DER as specified by IEEE Std 1547 is 10 MVA or less at PCC interconnected to the area EPS [30].

A very stringent requirement is the voltage limits at the PCC with 5\% maximum permissible deviation from normal grid voltage as specified in ANSI C84.1 [30]. For the purpose of this research, the utility-interactive inverter will be considered as the interfacing technology for a non-rotating primer mover technology such as a PV system.

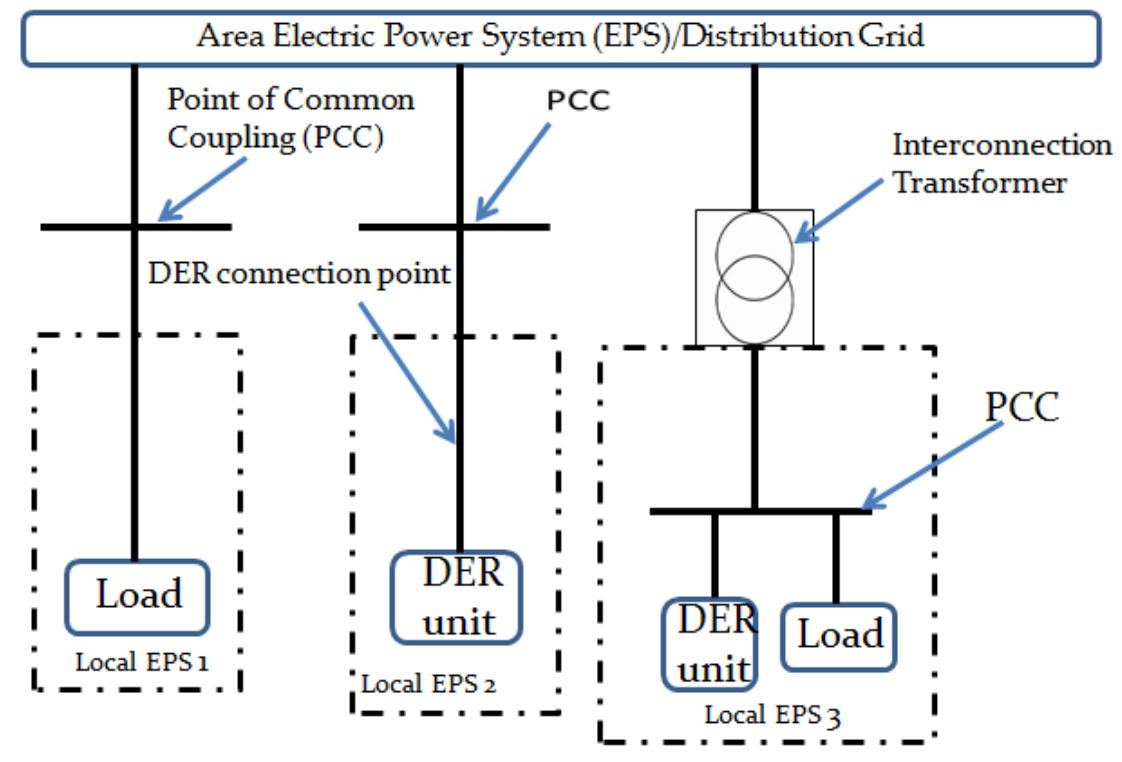

Figure 2.2: DER interconnection with the EPS

\subsection{Overview of Grid-tied Inverter Technology}

The deployment of utility-interactive inverter as an interfacing technology has grown in the recent times due to the evolution in the EPS with a special interest in incentivized PV systems [35], [36], [37]. For a non-rotating 
prime mover technology such as the PV system, a static power converter (inverter) is used as the interfacing technology to grid. It converts a nonsynchronous direct current (DC) from the PV unit to a synchronous ac (alternating current) voltage that can be interconnected smoothly with the distribution network [30], [33].

An overview of the inverter as a sub-system of the grid-connected PV is presented to understand its operational characteristics. Figure 2.3 shows the various configurations for grid-interactive inverter. In the topmost configuration, which is used in this research, a maximum power point tracking (MPPT) algorithm is implemented in the DC/AC converter to find the MPP of the PV panel. The middle configuration shows an intermediate DC/DC stage used to control the voltage output in order to obtain the peak available power from the PV system. In the last configuration, an energy storage system is connected in the DC stage in order to adjust the amount of energy supplied to the grid [33].

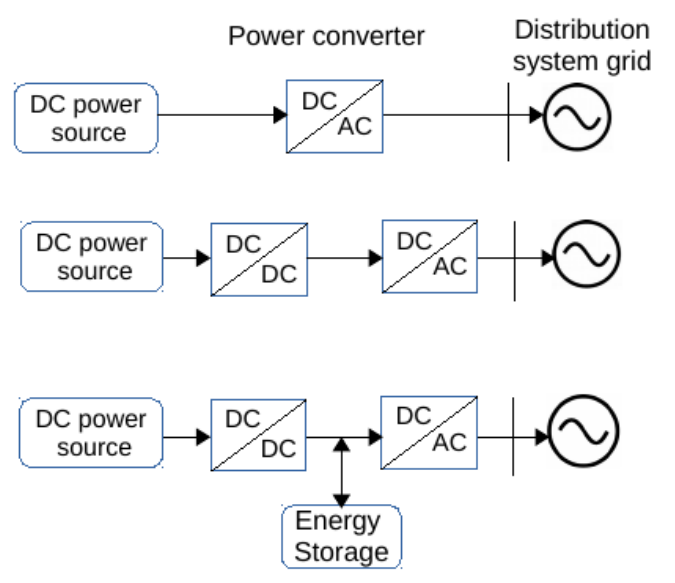

Figure 2.3: Various configurations for utility-interactive inverter

The voltage source inverter is the most commonly used interfacing technology to connect power sources to the grid. It uses self-commutating switches such as the insulated gate bipolar transistors (IGBTs) for power transfer to the grid [30], [33]. These inverters must be able to transfer op- 
eration from grid-tied to islanded mode and vice versa seamlessly to mitigate the impact of inherent variability of PV source [38].

Moreover, this thesis assumes that the grid supplies power during non or low sun hours which occurs most times in the early morning and night periods. Therefore, energy storage systems such as battery units are not required. Also, during peak sun hours where there is excess power generated from the PV system, the grid serves as a storage system and provides power when local generation is less than the load demands.

\section{Power Flow Analysis with PV-DG}

An inductively coupled inverter is used to interface PV-DG at the frontend to the distribution system as shown in Figure 2.4, usually as a current source with the stiff utility voltage [20], [30], [33], [39].

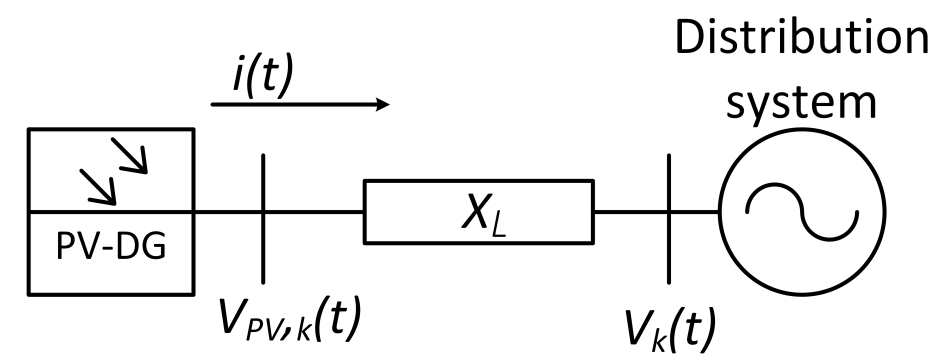

Figure 2.4: PV inverter connection to grid

For the distribution system with grid voltage $V_{k}$ at node $k$ given as [30], [33], [39]:

$$
V_{k}(t)=\left|V_{k}\right| \sin (\omega t)
$$

where $\omega$ is the grid voltage angular frequency.

The inverter output voltage, $V_{P V, k}(t)$, is given as:

$$
V_{P V, k}(t)=\left|V_{P V, k}\right| \sin (\omega t+\phi)+\sum_{h=2}^{\infty} v_{h c}
$$


where $\phi$ represents the power angle by which $V_{P V, k}(t)$ leads $V_{k}(t)$ and $v_{h c}$ is the voltage output harmonic components. Neglecting resistance, the active power supply from the PV-DG, $P_{P V, k}$, is given as:

$$
P_{P V, k}=\frac{\left|V_{P V, k}\right|\left|V_{k}\right| \sin (\phi)}{X_{L}}
$$

where $X_{L}$ is the addition of the inductance filter and leakage inductance of the interconnecting distribution transformer. The active power $\left(P_{P V, k}\right)$ supply to the grid can be controlled by varying the current injection $\mathrm{i}(\mathrm{t})$ to the grid since $V_{P V, k}$ is kept constant.

$$
i(t)=i_{f}(t)+i_{h c}(t)
$$

where $i_{f}(t)$ represents the steady state current (fundamental component) and $i_{h c}(t)$ is the higher harmonic current component. Also, in terms of voltage, $\mathrm{i}(\mathrm{t})$ injected from the PV-DG to distribution grid is given as:

$$
\begin{gathered}
i(t)=\frac{\int\left[V_{P V, k}(t)-V_{k}(t)\right] d t}{X_{L}} \\
i_{f}(t)=\frac{\int\left[\left|V_{P V, k}\right| \sin (\omega t+\phi)-\left|V_{k}\right| \sin (\omega t)\right] d t}{X_{L}} \\
i_{h c}(t)=\frac{\int \Sigma v_{h c} d t}{X_{L}}
\end{gathered}
$$

Alternatively, for a given inductively coupled inverter, active power supply to the grid control is accomplished by varying the power angle $\phi$, as given in [30], neglecting resistance:

$$
P=\frac{V_{P V, k} V_{k} \sin (\phi)}{X_{L}}
$$

Active Power is exported to the grid whenever inverter output voltage $V_{P V, k}$ leads the grid voltage $V_{k}$. The amount of active power injected into 
grid depends on the efficiency of the inverter and PV unit output power [30].

However, the previous versions of IEEE 1547 and UL 1741 in 2003 recommend that PV inverter shall not actively regulate voltage at the PCC. However, IEEE 1547a-2014 as an amendment states that "with approval from the concerned utility, DER units can now be allowed to be actively involved in voltage regulation by real and reactive power variation" [40]. The limitations provided by these standards depends on agreement with the concerned utility, which defines the operating conditions of DERs within the distribution network to maintain grid reliability. These two limitations (unity and non-unity power factors) will be considered for impact analysis in this study.

Therefore, the reactive power supply to the grid at node $k$ from the inverter, $Q_{P V, k}$, is calculated as:

$$
Q_{P V, k}=\frac{\left|V_{k}\right|}{X_{L}} *\left[\left|V_{P V, k}\right| * \cos (\phi)-\left|V_{k}\right|\right]
$$

Alternatively, $Q_{P V, k}$ is given as [41]:

$$
Q_{P V, k}=\lambda_{k} P_{P V, k}
$$

where:

$\lambda_{k}= \pm \tan \left[\cos ^{-1}\left(P F_{p v, k}\right)\right]$, positive $\lambda_{k}$ indicates the PV-DG is supplying reactive power and negative for absorbing reactive power, and $P F_{p v, k}$ is the PV-DG operating power factor at bus $k$.

\subsection{Related Work on Utility-interactive Inverter}

Wasynczuk [42] presented a three-phase line-commutated grid connected PV inverter system with its associated control used in transient stability programs. In another study, Wasynczuk et al. [43] described a simplified computer model of a three-phase self-commutated PV inverter used 
to predict the system behaviour during variation in irradiance and step changes in the inverter delay angle. The model produces a reasonably accurate result when compared with the detailed model based on valveby-valve detail. Also, wang et al. [44] used a line-commutated inverter to investigate transient and steady-state performance of a grid connected PV. The operating features and dynamic behaviour of PV system using single and three phase line and self commutated inverters were investigated in [45], [46].

Moreover, self-commutated inverters when compared with line commutated inverters have merits which include absence of commutator failure, independent control of active and reactive power, and black-start ability. However, line-commutated inverters are used for high voltage applications [47]. More so, since it is required that converters should remain connected even under abnormal voltage fluctuations, Bianchi et al. [48] proposed a multi-variable optimal control with anti-windup compensation to enhance performance during severe voltage perturbations. In [49], a single-stage grid connected inverter was presented with an ability to track the maximum power from the array and has advantages such as compact size, higher efficiency and optimization of PV array. A costeffective single-stage grid-tied inverter with maximum power tracking (MPPT) and one-cycle control (OCC) was demonstrated in [50]. The OCC has the capacity to control the output current and guarantees that it matches the grid requirements. Armstrong et al. [51] presented an auto-calibrating dc link current detecting method which removes current transducers errors and avoids dc current injection into the grid when using inverter systems without a transformer connection.

As reported by Kjaer et al. [52], grid-connected inverters are classified based on four metrics, which are:

- The number of power conditioning stages in cascade. 
- Power decoupling between PV unit(s) and single-phase grid type.

- Transformer or transformerless connection.

- Grid interfaces types.

\subsection{Impacts of utility-interactive PV systems}

The transition of the distribution system from a passive network to an active one as a result of PV penetration has resulted in significant changes in the design, planning and operation of the network [32]. The penetration of PV systems into the traditional power network could either enhance or degrade the power system performance depending on the planning and interconnection impact studies [53].

However, with a careful engineering, the potential adverse impacts such as system collapse, exposure of utility's asset and customer's equipment to damage, and reduction in reliability can be effectively mitigated [32]. The operational issues caused by PV-DG integration on distribution networks include the following [4], [30], [32], [53], [54]:

- Voltage impacts: The introduction of PV-DG can cause voltage rise, drop, fluctuations or unbalance out of limit, as specified by IEEE Std 1547-2003 and ANSI C84.1 (0.95-1.05 p.u.), depending on the deployment scenario.

A drop in voltage could occur if the PV system is installed just downstream a regulator with a line-drop compensator. Under heavy load conditions, the regulator may not raise the system voltage as much as required due to the power supplied by the PV-DG . Low voltage may also occur when the PV-DG is absorbing lagging reactive power from the grid.

Voltage rise occurs under a minimum load condition with a reverse power flow. Also, there is a possibility of system voltage increasing 
beyond the standard range if the PV-DG is injecting active and leading capacitive power when the primary feeder voltage is near the upper limit.

The inherent variability of PV-DG can cause voltage fluctuations especially on feeders with low stiffness ratio or factor. This factor is usually calculated at the PCC and measures the relative strength of the grid compared with the PV-DG. It is used to determine the ability of the grid to resist voltage fluctuations caused by the introduction of PV-DG.

Single-phase PV-DG can also result in voltage unbalance by injecting power only on one phase of the grid which causes an unbalance between the three-phase voltages. It is possible for the grid to experience voltage unbalance even if the phase voltages are within the specified range with PV-DG integration.

- Reverse power flow: High penetration of PV-DG during light load conditions can result in reverse power flow at section, substation transformer and feeder levels. Some fault conditions can also create insignificant reverse power flows which has to be sensed and cleared by system protection devices. This situation usually affect the operations of voltage regulators and over-current protection devices because their traditional designs only allow a unidirectional power flow. The integration of PV-DG within the hosting capacity of the feeder will help to avoid the occurrence of reverse power flow.

- Power losses: These are mostly ohmic in nature and power flow through loads, line branches and transformers are estimated as a function of current. There is strong correlation between power losses and equipment loading because they depend on the rms value of the current. PV-DG interconnection with grid can either increase or decrease power losses depending on factors such as location and size or penetration level. Power losses tend to decrease at a low or mod- 
erate penetration level depending on the feeder capacity to host PVDG. However, they increase at high penetration levels during light load conditions.

- Interaction with voltage regulating devices: The integration of a fluctuating source such as the PV-DG can affect the normal operations of voltage regulating devices such as voltage regulators and capacitor banks. Voltage fluctuations and rise from PV-DG can increase the on-load tap changer operations and on-off switching of voltagecontrolled capacitor banks. The frequent operations of these devices shortens their life span, and increases maintenance requirements and costs. These effects can be minimized by modifying the delay settings on the regulating devices to enhance coordination with the PVDG.

- Equipment overloading: The conventional positive-sequence source, which follows from a centralized power generation with voltage ramped-up on the transmission line and stepped down to a consumable voltage at the distribution end for end-use loads, is now altered with PV-DG. This reduces higher voltages towards the enduse loads, which decreases the risk of overload at these voltages. An overload of power delivery elements is avoided provided that the maximum power flow after PV-DG connection is less than the basecase. However, it is important that feeder sections connecting PV to the grid have ample capacity to distribute excess power generated by PV-DG. Also, re-conductoring of feeder sections may become necessary to accommodate high PV-DG penetration.

\subsection{Related work}

Several studies have been conducted on impact analysis of PV-DG integration with the distribution system. A review of these researches are pre- 
sented in this section.

\subsubsection{Voltage impacts}

Rylander et al. [55] presented voltage response of two feeders (with similar features in terms of voltage class and maximum load) to PV systems at different penetration levels before the hosting capacity was violated. Rylander et al. showed that each feeder has a unique response to PV systems penetration, which also depends on the inverter's power factor, PV size and location characteristics. Shahnia et al. [56] proved that PV systems have the capacity to either increase or decrease voltage unbalance depending on PV size, location and the load of the phase where the PV system is interconnected. Also, the study showed that rooftop PV installations in the low voltage distribution network will have a more severe voltage unbalance impact at the end of the feeder than at the beginning.

Liu et al. [57] investigated the effects of various PV-DG penetration levels on the voltage profile and traditional voltage regulation devices. Also, in the study the load buses were modelled as PQ buses. At high penetration levels $(30-50 \%)$, Liu et al. reported overvoltage in the secondary circuits with PV inverters capable of replacing capacitor banks used for voltage support along the feeder. An assessment on voltage profiles within residential neighbourhoods with high penetration of PV systems was presented by Tonkoski et al. [58]. It was shown that in order to determine the voltage rise rate, certain factors such as feeder impedance, transformer impedance, feeder length and penetration levels up to $11.25 \%$ are to be considered. Also, overvoltage problems can be mitigated by reducing transformers short circuit resistance and feeder impedance. Weckx et al. [59] reported that the voltage unbalance factor can be reduced up to $40 \%$ on average for each bus by deploying optimally controlled balancing inverters.

Thomson et al. [60] reported network voltage rise as a result of wide 
spread PV generation on the low voltage network using load and generation data at 1 minute intervals. The study showed that for a typical urban network, a high PV penetration (up to 50\%) will only lead to small increase in average distribution network voltages. Also, Katiraei et al. [4] showed that network voltage rise and fluctuations are more evident with PV systems interconnected at the rear of long lightly loaded feeders. This effect can be mitigated by operating PV inverters at non-unity power factors and switching off capacitor banks during PV peak output. In addition, voltage rise and fluctuations (due to cloud passage) at 20\% penetration level due to randomly distributed PV system was demonstrated by Smith et al. [61].

Moreover, Hoke et al. [62] investigated the peak PV penetration relative to steady-state voltage and overcurrent for different deployment scenarios. The maximum penetration for distributed PV systems was almost always above $50 \%$ with the exception of cases where the feeder had shown peak load voltages on the ANSI C84.1 range A upper limit without PV. The PV system model in this study used an hourly solar irradiance data and also, neglected the effect of temperature on PV-DG performance. Hung et al. [41] used time-varying voltage-dependent load models to determine the penetration level of PV units using an average hourly solar irradiance data.

\subsubsection{Impact on power losses}

Thomson et al. [60] presented an aggregated low voltage network losses and average transformer loading reduction for a typical urban network as the PV penetration level increases from 30 to 50\%. The loss reduction was not proportional with penetration levels because of the large amount of reverse power flows at $50 \%$ penetration, and the transformer loading reduced during sun hours. Weckx et al. [59] presented the reduction of distribution grid losses by the installation of two balancing inverters, with central and local controllers. The central control considered the voltage 
drop over the line which depends on the line current while local control used the absolute values of the local voltage measurements. As a result of the optimal control, the distribution grid losses were reduced by approximately $10 \%$.

Quezada et al. [63] investigated the impacts of various DGs (such as PV and wind systems) and showed that system losses decrease with small amount of DG penetration until a minimum value is reached, and then starts to increase with rising penetration level with losses higher than the basecase. The study also, reported that voltage and reactive power control, whether injected or consumed by the PV inverter can reduce energy losses. Studies in [64], [65] showed that poor or non-optimal location and size of a DG system can increase power losses.

\subsubsection{Impact on voltage regulation devices}

One of the pioneering attempts to show the impact of PV penetration on the distribution system voltage regulation device is presented in [66]. Garret et al. [66] developed a model that showed the switching operations of the voltage regulation device (90 operations) with the integration of a relatively large PV system with an average hourly typical meteorological year (TMY) data. Kern et al. [67] investigated the PV generation (56 $\mathrm{kW}$ ) effects on a $13.8 \mathrm{kV}$ distribution feeder supplying 28 PV-equipped houses in Gardner, Massachusetts. The study showed that there was no problem with voltage regulation but with a tendency to cause increased number of operations of the transformer tap changer. Also, Hariri et al. [68] used Quasi-Static Time Series (QSTS) simulation to show that when the PV inverter system is allowed to participate in voltage regulation, the voltage regulator operations might increase and therefore, makes updating the traditional system controls a pivotal requirement. The study used a synthesized one minute resolution irradiance data for simulation and analysis. 
Ravindra et al. [69] compared the impact of a single large PV plant and distributed systems on the operation of the OLTC with a one minute resolution irradiance data. The study revealed that distributed PV-DG with voltage control caused more tap operations that the large PV plant. Agalgaonkar et al. [70] presented a control strategy to mitigate the negative impact of the PV plant on the operation of the on-load tap changer (OLTC) and voltage regulator (i.e., the increased number of operation and runaway issues) using a solar irradiance profile at 30-s resolution. Ari et al. [71] studied the impact of $20 \%$ PV penetration on a simple distribution network using one minute irradiance at a single point. Ari et al. also reported a significant increase in tap operations even with a five-minute delay. For utilities envisaging numerous adverse effects on voltage regulation devices when integrating a large PV plant, Katiraei et al. [4] proposed an express (dedicated) feeder to connect the plant directly to the substation.

In order to minimize the problem of excessive operations of voltageregulating devices, IEEE Std 1547.2-2008 recommends the following [30]:

- Modification of the time delay settings on the regulating devices in order to provide an improved coordination with the DG unit.

- For severe cases, the installation of static VAR compensation may be needful.

- Coupling DG unit with a variable source (another DG unit) that has the capacity to dampen or "flatten" out the fluctuations could mitigate the increased operation of the regulating devices.

\subsection{Gaps in the related work}

The utility leadership and planners still express a lot of scepticism with PV-DG integration in the distribution system due to the fact that some 
pivotal characteristics of the solar resource have not been fully considered in most interconnection studies. For instance, the time-varying nature of the solar irradiance at various resolutions and its interaction with the grid. This is a key feature in assessing the true impact of PV-DG integration on the grid.

The extant literature reports grid-connected PV-DG modelling with average hourly data from a single-point irradiance sensor. The variability observed from a single point irradiance sensor cannot give the exact representation of variability across the entire PV plant. The use of TMY irradiance data could be useful for energy production estimations, however, such data usage suffers a major limitation in grid integration studies because it does not account for cloud induced fluctuations. This particular constraint is a major concern to utility and system planners in assessing the true impact of PV-DG interconnection.

Generally, the data resolution required depends on the type of analysis that is carried out. For steady state overvoltage simulations, minutes data resolution is recommended, while hourly and seconds-minutes can be used for energy and voltage fluctuations respectively [61]. Also, the use of single-point irradiance data without geographic smoothing over the entire PV plant footprint will affect the accuracy of the grid-connected PV modelling with its associated impacts on the power system. It is apparent that interconnection studies that consider geographic smoothing is required to accurately model utility-interactive PV-DG.

In addition, the existing study have performed integration of PV-DG with the inverter interface operating at a fixed power factor. The possibility of scheduling the power factor with respect to PV output and sun hours can help to maximize the value of PV plant while minimizing the adverse impacts. This erases the assumption of a particular amount of PV output each day and reduces the possibility of violating system constraints.

Also, although power loss reduction as a result of PV-DG integration has been largely reported in the literature. However, the components of 
power losses which are load, line and transformer losses have not been investigated which are necessary for effective distribution system design. Further, most of the studies have neglected the local impacts of PV-DG integration such as increase in bus voltages which can be propagated to the entire system and thereby, affecting system stability.

The intrinsic strong correlation that exists between distribution network capacity and reliability gives the inclusion of renewable DG and the evaluation of their additional load serving capacity a prime place in the emerging active distribution system planning. The existing literature has not reported the incremental capacity addition provided PV-DG based on power delivery system limits, and its computation which is very vital for utilities. The measurement of risk and upgrade deferral with PV-DG deployment are now considered pivotal especially as load demand continues to outpace new feeder construction.

DG power models for power flow simulations are very important considerations for effective and efficient modelling of PV-DG in the distribution network. The performance of these models are essential in maximizing the value of PV-DG while minimizing the adverse impact. Interconnection studies are currently required to compare the performance of these models for active distribution analysis.

\subsection{Relevant PV-DG interconnection standards}

A portion of this section has also been peer-reviewed and published in [72].

It is important to maximize the full value of PV-DG such as grid capacity expansion, provision of environmentally responsible energy and ancillary services while retaining power network reliability. However, this is possible only by a strict adherence to common functional technical requirements and specifications relevant to the effective operation and performance of the next-generation grid. The relevant standards are listed 
below [30], [73]:

1. IEEE 1547 series of standards: The popular IEEE 1547-2003 suite of standards and 2014a amendment 1 continue to underpin the development of the DER technologies integration with the EPS. They provide guidelines for conformance testing, monitoring and controlling, design and operation, and conducting distribution impact studies of DER interconnection with the grid. The standard series are as follows :

- IEEE Std 1547.1 (2005): IEEE Standard Conformance Test Procedures for Equipment Interconnecting Distributed Resources with Electric Power Systems.

- IEEE Std 1547.2 (2008): IEEE Application Guide for IEEE Std 1547, IEEE Standard for Interconnecting Distributed Resources with Electric Power Systems.

- IEEE Std 1547.3 (2007): IEEE Guide for Monitoring, Information Exchange, and Control of Distributed Resources Interconnected with Electric Power Systems.

- IEEE Std 1547.4: Draft Guide for Design, Operation, and Integration of Distributed Resource Island Systems with Electric Power Systems.

- IEEE Std 1547.5: Draft Technical Guidelines for Interconnection of Electric Power Sources Greater Than 10 MVA to the Power Transmission Grid (Withdrawn December 2011).

- IEEE Std 1547.6 (2011): Draft Recommended Practice for Interconnecting Distributed Resources with Electric Power Systems Distribution Secondary Networks.

- IEEE Std 1547.7 (2013): Draft guide to Conducting Distribution Impact Studies for Distributed Resources Interconnection. 
- IEEE Std 1547.8: Draft Recommended Practice for Establishing Methods and Procedures that Provide Supplemental Support for Implementation Strategies for Expanded Use of IEEE Std 1547.

Further, a major part of the amendment (IEEE 1547a-2014) now permits DER to be actively involved in voltage regulation via real and reactive power variation under a mutual agreement between the utility and DER operators. Moreover, in conjunction with IEEE 1547.1 is the supplementary standard, Underwriters Laboratories (UL) 1747, which provides requirements for inverters, converters and controllers used as grid interfaces for grid-connected DERs.

2. IEEE 1547 Std 2030: IEEE Guide for Smart Grid Interoperability of Energy Technology and Information Technology Operation with the Electric Power System (EPS), End-Use Applications, and Loads.

3. IEC 61727: PV systems - Characteristics of the Utility Interface.

4. IEC 62116: Utility-interconnected PV Inverters - Test Procedure of Islanding prevention.

5. IEC 61724: Photovoltaic System Performance Monitoring Guidelines for Measurement Data Exchange and Analysis

\subsection{Research strategy}

This section summarizes the findings and gaps from the literature with the proposed contributions.

Several case studies and performance analysis of various PV technology types deployed in different countries are reported in the literature. However, no New Zealand case has been documented in IEA PVPS reports and other reviewed literature. This thesis investigates the performance and the economic viability of a monocrystalline (m-Si) grid-connected 
PV system in New Zealand using a real load demand data. The performance of the m-Si PV technology is compared with similar and other technology types deployed under diverse climatic conditions. Also, this research confirms the significant impact of temperature on the overall performance of the PV technology. The analyses presented provides investors, prospective prosumers, policy makers and other stakeholders with the required knowledge in promoting the full adoption of PV system technology as an emerging sustainable power generation resource.

The literature reports the impact of single-phase PV systems on voltage profiles, power losses and voltage unbalance. Also, low data resolution (10-minute and hourly irradiance) was used to simulate these impacts on the grid. Feeder equipment loading, bus voltage and component of power losses impacts with computational costs have not been reported in the literature. Further, the extant literature has generalised the operations and impacts of DG models for all types of DER in the distribution system. This thesis analyses the impact of spatially distributed single-phase PV systems on the distribution network using a typical school load profile and solar irradiance data with a five-minute resolution. This research analyses and compares the performance of the DG models in terms of the load buses voltage profiles and unbalance, feeder equipment loading, components of power losses and the computational cost required for a converged power flow solution. In addition, this study investigates the impact of these DG models for single-phase PV systems integration with the grid.

Moreover, the extant literature has extensively used effective load carrying capacity (ELCC) to compute the capacity contribution of a new generator. However, there are several concerns regarding ELCC calculations such as the use of a constant annual reliability level without taking into account the possibility of peak load reliability changing and the impact of the DG on the loadings of the power delivery elements. Also, it is a probability based measure, exaggerates capacity contribution at peak load and requires huge data with a non-trivial computational requirements. This 
study proposes metrics capable of computing the incremental capacity additions, measuring risks and upgrade deferral provided by PV systems deployments. These indices include, energy exceeding normal (EEN) rating and unserved energy (UE), for evaluating system design reliability and capacity usage. Also, sensitivity analysis for optimal siting and generation of power loss variation curves for optimal sizing are implemented. This is very crucial because of the intrinsic relationship that exists between network capacity and reliability.

The extant literature models PV variability with low resolution (averaged 1-minute and hourly) and single-point irradiance data. PV-inverter interface has been deployed with a fixed power factor and very limited reporting on the impact of PV variability on $\mathrm{kW}$ and kVar profiles. It is important to note that short-time effects such as the tap changing operations are masked with low resolution data. Also, the variability observed from a single-point sensor cannot accurately represent the variability across the entire PV plant foot print. Furthermore, cloud-induced fluctuations which significantly impact the grid adversely and other key factors such as the PV density and geographic smoothing for modelling PV variability are omitted in the literature. This thesis uses the upscaling advantage from a single module and point irradiance sensor to geographic smoothing over the entire PV footprint in wavelet variability model (WVM) to simulate effects of a grid-connected large scale PV system on the IEEE-34 distribution feeder. This research uses a high-frequency solar irradiance data (1 s) to show the PV plant locational value and its output variability impacts on voltage regulator tap changing operations, feeder voltage, active and reactive power profiles at various penetration levels until the voltage constraint is violated. Also, this thesis implements power factor control which is a part of the advanced inverter grid support functions to provide the grid with the needed voltage support and control over the PV plant output supply to the distribution network.

This research performs time-series power flow analysis to show how 
the time-varying nature of the load and PV profiles affect the operation of the network devices on the grid. Also, this type of analysis reveals the extent and frequency of study criteria violations.

\subsection{Summary}

This chapter presents the background and related work relevant to this study. The background covers the overview of the PV system output model with the inputs and various steps involved. A review of DER and its interconnection requirements with the distribution system have been provided. Also, since this study focuses on the integration of PV technology as a typical example of DER. A review of the operational local and system-wide impacts of integrating PV-DG on the distribution network is presented.

This section highlights the limitations of the existing interconnection studies approaches such as the irradiance data resolution from a single point sensor without geographic smoothing. It is clear that studies on PV-DG variability modelling and incremental capacity computation are required for the graceful adoption of PV system as a power generating resource for the evolving smart grid. The last part of this chapter discuses DG interconnection requirements and standards.

\subsection{Next chapter}

The next chapter presents an evaluation of the operational and reliability performance of a grid-connected PV system as a case study. This provides an understanding of the operation of PV-DG with the assessment of various performance indices such as energy yield, performance ratio, levelized cost of energy and simple payback time. The analysis presented in the chapter will help to assess PV credibility, viability, detect design 
defects early and increase its penetration in the traditional electric power system. 
Chapter 2 


\section{Chapter 3}

\section{Performance analysis of utility-interactive PV systems}

This chapter presents a performance analysis of a grid-connected solar photovoltaic (PV) system installed at Maungaraki school, Wellington, New Zealand. Also, a simple economic analysis based on the PV power output of the system is presented in this chapter. The techno-economic analysis provides a basic understanding of the technical feasibility and economic viability of such PV systems. In addition, the metrics presented in this chapter allow a cross-comparison for PV systems operating under diverse environmental conditions. The results of this study have been peerreviewed and published in [19].

\subsection{Introduction}

The proliferation of grid-connected PV systems on the electricity grid highlights the need to understand its operation through monitoring and thorough performance analysis [74]. Also, the utility-interactive PV-DG is essentially monitored to evaluate the final energy yield, detect possible design defects and avoid economic losses as a result of operational issues [74]. Another reason to carry out such analysis is to assess the PV systems 
credibility, viability and ultimately to increase their penetration within the existing electric power network [75].

The IEA PVPS Task 2 has been able to analyse and publish 170 gridconnected PV systems installed in various countries of the world [76]. In addition, over the previous years, various authors from different countries have published results of the performance analysis of their respective grid-connected PV units. Pietruszko et al. [77] evaluated the performance of a $1 \mathrm{kWp}$ a-Si PV system located at Warsaw, Poland. The performance ratio ranged from 0.6 to 0.8 , annual system yield was $830 \mathrm{kWh}$ and the efficiency of the PV system was in the range of $4-5 \%$. Mondol et al. [78] presented the outcome of the performance analysis of a $13 \mathrm{kWp} \mathrm{m-Si} \mathrm{PV}$ system installed in Northern Ireland. The evaluated annual final yield ranged from 1.61 to $1.76 \mathrm{~h} / \mathrm{d}$, with a PR which ranged from 0.6 to 0.62 and annual average system efficiency of $6.4 \%$. Also, Chokmaviroj et al. [79] evaluated the performance of a $500 \mathrm{kWp}$ grid-connected PV plant at Mae Hong Son province, Thailand. The plant was divided into two, $250 \mathrm{kWp}$, from a double glazed semi-Si PV modules. The plant generated about $383274 \mathrm{kWh}$ and the efficiency of the PV array ranged from 9 to $12 \%$. The final yield ranged from 2.91 to $3.98 \mathrm{~h} / \mathrm{d}$ and the PR ranged from 0.7 to 0.9 .

Another performance study is that of Kymakis et al. [80]. The study evaluated a p-Si $171.36 \mathrm{kWp}$ utility interactive PV park on the island of Crete, which was as a result of the favourable climatic condition and the recent incentivization of PV system installations in Greece. The yearlong evaluated PR was 0.67 and the final yield ranged from 1.96 to $5.07 \mathrm{~h} / \mathrm{d}$. Cherfa et al. [81] carried out an analysis of a mini-grid connected $\mathrm{m}-\mathrm{Si}$ $9.54 \mathrm{kWp}$ PV system which was a pilot project with the primary aim of acquiring experience in the design, monitoring and maintenance of such innovative technology in Algeria. The system performance was quantified over the monitored period which showed an annual $10981 \mathrm{kWh}$ of energy injected into the grid. The average daily output energy was $30 \mathrm{kWh}$ and PR ranged from 0.62 to 0.77 . 
Additionally, the analysis of the economic viability of utility-interactive PV systems is very crucial in enhancing PV technology uptake as favourable renewable energy policies continue to evolve globally. Adaramola [82] presented the viability of grid-connected PV system in Jos, Nigeria. The study showed that the $80 \mathrm{kWp}$ system was able to contribute $40.4 \%$ of the annual electricity demand with LCOE of $\$ 0.103 / \mathrm{kWh}$. Mondal et al. [83] presented the financial viability of a proposed 1-MWp grid connected PV system in Bangladesh. For a project lifetime of 15 years, Mondal et al. reported energy production cost between $\$ 0.253 / \mathrm{kWh}$ and $\$ 0.282 / \mathrm{kWh}$, which decreases with the increase of lifetime. Also, El-Shimy [84] presented a viability analysis of a $10 \mathrm{MWp}$ grid-connected PV plant for 29 different sites in Egypt. The energy production cost ranged from $\$ 0.1989 / \mathrm{kWh}$ to $\$ 0.2424 / \mathrm{kWh}$, and the equity payback varied between 4.9 years and 7.1 years. Adaramola [85] analysed the economic viability of a rooftop 2.07 $\mathrm{kW}$ grid-connected PV system in Norway with a feed-in-tariff of $\$ 0.356 / \mathrm{kWh}$ over 25 years of project lifetime. This resulted in $\$ 0.110 / \mathrm{kWh}$ premium over the LCOE of $\$ 0.246 / \mathrm{kWh}$ produced by the PV system.

However, performance analysis of grid-connected PV systems in New Zealand are not available in the literature which is pivotal in understanding and quantifying their impacts on the traditional electricity network as an emerging alternative renewable power generation resource. Also, in order to develop the PV system as a sustainable energy resource and increase its uptake, it is important to assess its economic viability.

This study aims to fill these gaps by presenting a techno-economic analysis of a grid-connected $10 \mathrm{kWp}$ PV system at Maungaraki school, Wellington in New Zealand as a case study. Evaluation is carried out based on performance parameters specified by IEA PVPS and IEC 61724 standard for utility-interactive PV systems. In addition, the Clean Energy Council (CEC) guide which represents the industry best practice for the design and installation of grid-tied PV systems has been used for analysis in this study. 
Final system yield, energy yields, capacity factor and performance ratio are evaluated. Also, the economic viability of the PV system under consideration has been evaluated using metrics such as the NPV, LCOE and simple payback period. In addition, results from the Web Based PV System Monitoring and Reporting (WBPSMR) for the PV array are presented.

\subsection{Description of the grid-tied solar PV system}

The installation of the $10 \mathrm{kWp}$ PV array was carried out under the "Dynamis Project" with the aim of testing renewable energy technologies effectiveness and economic viability using schools as platforms in order to sensitize surrounding communities to become more energy self-sufficient [86]. The renewable energy for New Zealand schools under this project has successfully installed $10 \mathrm{kWp}$ solar PV in two different schools. One of the installations was carried out at Maungaraki school (with a roll of about 250 pupils), as an attempt to pioneer a sustainable energy revolution. The school is located at a longitude of $174.9^{\circ} \mathrm{E}$ and latitude of $41.16^{\circ} \mathrm{S}$, with a panel tilt angle of $41^{\circ}$. The PV panel tilt angle and the latitude of the corresponding site location are kept equal in order to obtain maximum solar radiation [87], [88].

There are two identical parallel strings consisting of 20 panels each with modules rated at $250 \mathrm{Wp}$ capacity, and tied to the grid via two $5 \mathrm{~kW}$ Enasolar single-phase inverters (as shown in Figure 3.1). Also, the inverters deployed have two independent maximum power point tracking PV inputs which can be connected to one solar string or two identical parallel solar strings [89].

In addition, the inverter has integrated lockable DC and AC switches for isolation purposes. The most relevant technical specifications of the inverter are presented in Table 3.1.

The energy meter is used to measure the amount of energy consumed 
Table 3.1: Electrical properties of the inverters.

DC Input

Number of inputs

Max. open circuit voltage $\left(V_{o c}\right)$

DC full power operating range

Operating voltage range $\left(V_{m p p}\right)$

DC optimal operating voltage

Max. input current $\left(I_{m p p}\right)$

Max. short circuit current $\left(I_{s c}\right)$

Maximum usable input power $\left(P_{\max }\right)$

Maximum allowable input power

Reverse polarity protection

AC Output

Nominal output voltage

Output voltage range

Output power (@50 Hz)

Max. output current

Power factor

Max. efficiency

Max. Euro. efficiency

General system data

Data interface

Weight

Night-time consumption
2X Independent MPPT inputs

600 per DC input

235-500 V per DC input

120-500 V per DC input

350V per DC input

15 A per DC input

$16 \mathrm{~A}$

3500 Wp per DC input

$7000 \mathrm{~W}$

Inherent crowbar diodes

$230 \mathrm{~V}$ AC single phase

202-259 V AC (New Zealand)

$4990 \mathrm{~W}$

$21.5 \mathrm{~A}$

$>0.98$

$>96.8 \%$

$>95.4 \%$

IEEE 802.11 (Wi-Fi)/Ethernet

$20 \mathrm{~kg}$

$<1.2 \mathrm{~W}$ 


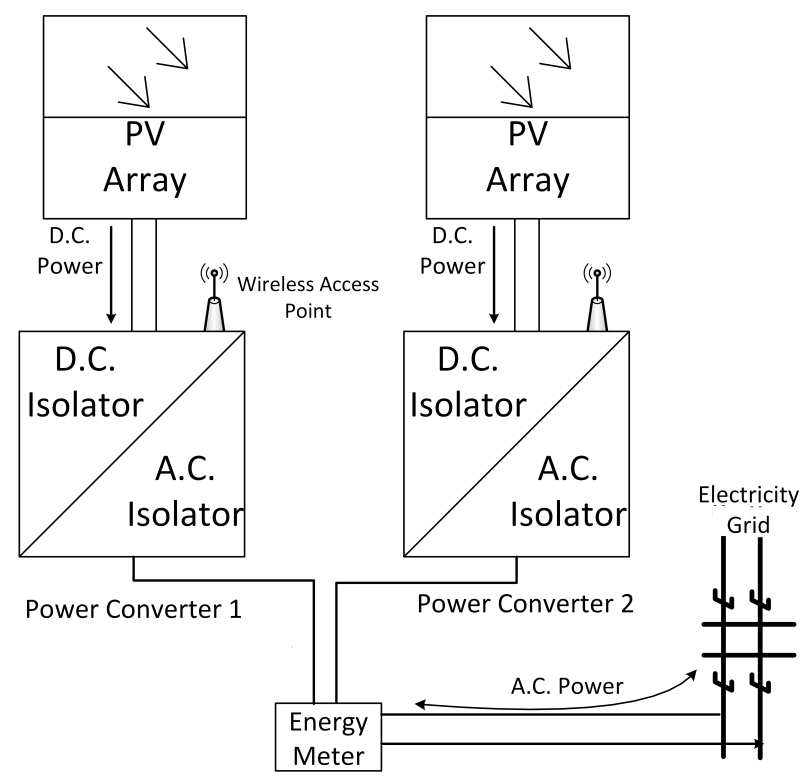

Figure 3.1: Schematic block diagram of the PV system

and the excess power generated and exported to the grid which usually occurs during minimum load conditions that happens over weekends and holiday periods. The specification of the TNS250 module used is given in Table 3.2. In addition, the PV array was installed in an open space on a rooftop free of shadows or shading which can affect its performance. The PV panels are regularly maintained every six months to get rid of dust, birds' drops and other forms of impurities. Also, the cost of setting up the project was about NZD \$28000 sourced through grants and donations.

\subsection{System Performance Analysis}

This study considers the evaluation of the operational and reliability performance of the grid-connected PV system based on the IEC standards (61836 and 61724) and reports from International Energy Agency (IEA) photovoltaic power systems program (Task 2) [76], [90], [91]. The performance analysis of grid-connected PV systems (as shown in Figure 3.2) 
Table 3.2: PV module specifications

\begin{tabular}{ll}
\hline PV module & Specifications \\
\hline Type of cell material & Monocrystalline \\
\hline Make & TNS Solar \\
\hline Model & TN-60-6M \\
\hline Maximum power $\left(P_{m a x}\right)$ & $250 \mathrm{Wp}$ \\
\hline Open circuit voltage $\left(V_{o c}\right)$ & $37.9 \mathrm{~V}$ \\
\hline Short circuit current $\left(I_{s c}\right)$ & $8.64 \mathrm{~A}$ \\
\hline Voltage at maximum power $\left(V_{m p}\right)$ & $30.4 \mathrm{~V}$ \\
\hline Current at maximum power $\left(I_{m p}\right)$ & $8.23 \mathrm{~A}$ \\
\hline Number of cells in a module & 60 \\
\hline
\end{tabular}

is pivotal in assessing their operational performance under different climatic conditions and detection of operational issues [19]. It also enables the measurement of the long-term variation in system performance and comparisons with other systems that differ in location, design and technology [75].

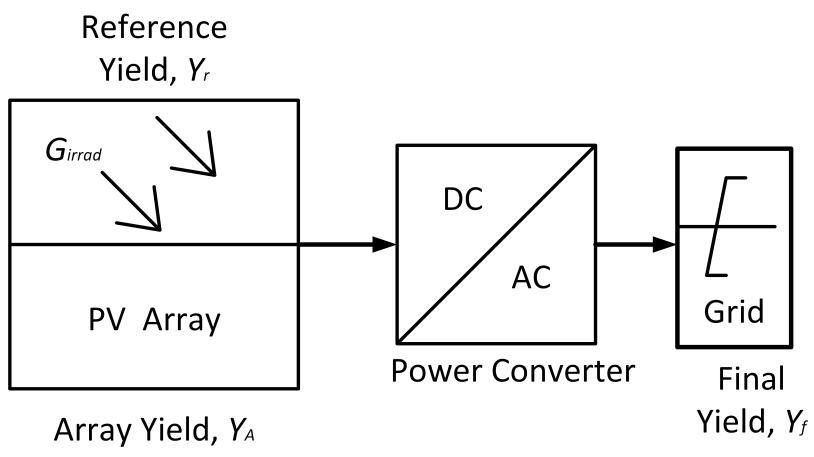

Figure 3.2: System performance analysis 


\subsubsection{Reference yield}

The reference yield, $Y_{r}$, is a function of in-plane irradiation and estimated as the ratio of the total irradiation per day (or year) to the reference irradiance [76], [90]. Therefore, the daily $Y_{r}$ is calculated as:

$$
Y_{r}=\frac{\sum_{t=1}^{24} G_{I}}{G_{I, \text { ref }}}
$$

where $G_{I}$ is the total irradiation $\left(k W h / m^{2}\right)$ and $G_{I, r e f}$ represents the reference irradiance $\left(k W / m^{2}\right)$.

\subsubsection{PV Array energy yield}

The ratio of the energy produced by the PV array $\left(E_{A}\right)$ to the rated PV capacity $\left(P_{O}\right)$ is referred to as the array yield, $Y_{A}$. Losses such as the ones due to manufacturing tolerance, temperature, dirt and dust are taken into consideration in estimating $Y_{A}$ [76], [90]. It is therefore, calculated as:

$$
Y_{A}=\frac{E_{A}\left[k W h_{D C}\right]}{P_{O}\left[k W p_{D C}\right]}
$$

\subsubsection{Final system yield}

The final system yield, $Y_{f}$, is the ratio of the net energy produced $\left(E_{N E T}\right)$ by the PV array to the rated DC array capacity $\left(P_{O}\right)$ [90]. Also, it is the amount of energy supplied to the load per day (month or year) considering array capture and system losses. It quantifies the duration (e.g., yearly, monthly or daily) required by the PV array to operate at the rated DC power to supply an equal amount of energy and estimated as [75], [80]:

$$
Y_{f}=\frac{E_{N E T}\left[k W h_{A C}\right]}{P_{O}\left[k W p_{D C}\right]}
$$




\subsubsection{Performance ratio}

This is a dimensionless quantity used to indicate the monthly or annual impact of PV system losses on the rated array capacity. The system losses are as a result of $\mathrm{PV}$ array temperature, failure of system components and incomplete usage of the irradiation [90]. In addition, the performance ratio, $P R$, does not depend on location of PV installation and system size [76]. It is used to evaluate the quality of PV system installation and estimated as [92]:

$$
P R=\frac{\text { PV system actual energy yield }}{\text { PV array ideal energy output }}=\frac{Y_{f}}{Y_{r}}
$$

\subsubsection{Losses}

System losses, classified into DC and AC subsystems losses, are pivotal design considerations in providing realistic energy solutions [93]. However, in this study, losses due to shading or shadows are not considered because the location of the PV system is free of shadows during the day.

The connecting cable (PV DC main cables) between the PV array and the inverter subsystems can lead to power loss which should be accounted for. For our case study, the DC cable losses is 3\% which amounts to 0.97 de-rating factor [75], [92]. However, AC subsystems losses is as a result of the connecting cable between the inverter and the grid with a typical value of $1 \%$ and de-rating factor of 0.99 [75], [92]. These two losses are components of the system losses, $L_{s}$, and estimated as:

$$
L_{s}=Y_{A}-Y_{f}
$$

The array capture losses, $L_{c}$, refers to the normalised PV system losses which is as a result of PV array energy losses such as conversion losses, manufacturing tolerance and dirt [90], [94]. In addition, $L_{c}$ is calculated as: 


$$
L_{c}=Y_{r}-Y_{A}
$$

\subsubsection{Capacity factor}

The capacity factor, $L_{S P}$, is the defined as the ratio of the energy output from the PV system to the product of the operating duration and rated PV array output [95]. $L_{S P}$ is calculated annually as [80]:

$$
L_{S P}=\frac{E_{N E T}}{P_{O} * 8760}=\frac{Y_{f}}{8760}
$$

\subsubsection{Inverter efficiency}

The inverter efficiency, $\eta_{i n v}$, is defined as the ratio of the AC power produced by the inverter $\left(P_{I n v, A C}\right)$ to the DC power generated by the PV array unit $\left(P_{P V, D C}\right)$ [96], [97].

$$
\eta_{i n v}=\frac{P_{I n v, A C}}{P_{P V, D C}}
$$

\subsubsection{System efficiency}

The monthly system efficiency, $\eta_{\text {sys }}$, is given as [98], [99]:

$$
\eta_{\text {sys }}=\frac{E_{N E T}}{G_{\text {irrad }} * A_{\text {array }}}
$$

Here, $E_{N E T}$ is the monthly total AC PV energy output, $G_{\text {irrad }}$ is the monthly peak sun hour, and $A_{\text {array }}$ is the area of the array.

\subsubsection{Energy yield}

Energy yield of a PV array is a function of the meteorological data of the location where it is installed [100]. This is the PV system energy output estimated as [92], [101], [102]: 


$$
E_{N E T}=P_{\text {rated-STC }} * \mu_{\text {temp }} * \mu_{\text {man }} * \mu_{\text {dirt }} * G_{i r r a d} * \eta_{i n v} * \eta_{p v-i n v} * \eta_{i n v-s b}
$$

where:

$$
\begin{aligned}
E_{N E T} & =\text { PV system energy yield }(\mathrm{Wh}) \\
P_{O} & =\text { rated DC output power of the array under standard test conditions }(\mathrm{Wp}) \\
\mu_{\text {temp }} & =\text { de-rating factor due to temperature } \\
\mu_{\text {man }} & =\text { de-rating factor for manufacturing tolerance }(0.97) \\
\mu_{\text {dirt }} & =\text { de-rating factor for dirt }(0.97) \\
G_{\text {irrad }} & =\text { irradiation value or peak sun hour }\left(\mathrm{kWh} / \mathrm{m}^{2}\right) \\
\eta_{\text {inv }} & =\text { efficiency of the inverter }(0.96) \\
\eta_{p v-i n v} & =\text { efficiency of the sub-unit between the PV unit and the inverter }(0.97) \\
\eta_{i n v-s b} & =\text { efficiency of the sub-unit between the inverter and the switchboard }(0.99)
\end{aligned}
$$

\section{Temperature de-rating factor}

The de-rating due to temperature for this study is done in accordance with guidelines provided by the Clean Energy Council (CEC) for gridconnected solar PV systems without battery storage [101]. The cell temperature $T_{C}$ for a grid-connected $\mathrm{PV}$ is estimated as:

$$
T_{C}=T_{A}+T_{R}
$$

where $T_{A}$ and $T_{R}$ represent ambient temperature $\left({ }^{\circ} C\right)$ and temperature rise for parallel-to-roof installation $\left(35^{\circ} \mathrm{C}\right)$ respectively.

However, $T_{R}$ is a function of PV array installation type which could be top-of-pole, parallel-to-top or rack-type mount [10]. The parallel-to-top array frames were deployed on site used for our case study with a $T_{R}$ of $35^{\circ} \mathrm{C}$ as recommended by CEC. With the estimated value of $T_{C}$, the derating factor due to temperature, $\mu_{\text {temp }}$, is given by: 


$$
\mu_{\text {temp }}=1+\left\{\lambda *\left(T_{C}-T_{S T C}\right)\right\}
$$

where $\lambda$ is the power temperature coefficient $/{ }^{\circ} \mathrm{C}\left(-0.45 \% /{ }^{\circ} \mathrm{C}\right)$ and $T_{S T C}$ represents the cell temperature at standard test conditions $\left({ }^{\circ} \mathrm{C}\right)$.

\subsection{Economic analysis}

The enhancement of the cost-competitiveness of the solar PV system over the years continues to drive its uptake as a viable power generation alternative in the global energy mix. In 2014, prices of PV modules decreased by $75 \%$ in comparison with their prices at the close of 2009 and as a result, improving PV system plug parity [103]. Important measures used in the economic evaluation of the PV system considered in this research are as follows:

\subsubsection{Net present value (NPV)}

The NPV is a financial tool used to evaluate cash outflows and revenues, and investment characteristics and decisions especially for comparing mutually exclusive projects [104], [105]. Also, it is the algebraic sum of the net cash flows over the project's life time to the present, discounted by an appropriate discount rate [106]. It is given given as:

$$
N P V=\sum_{m=0}^{N} \frac{C F_{m}}{(1+r)^{m}}
$$

where $C F_{m}$ is the net cash flow in year $m, N$ is the analysis period and $r$ the annual discount rate. The NPV criteria include [105], [107]:

- If the $N P V$ is positive, the investment is economical.

- Negative $N P V$ denotes the return are worth less than the initial investment. 
- The financial viability of a project is uncertain with Zero NPV.

- Amongst independent projects, the higher the $N P V$ value the better.

\subsubsection{Levelized cost of energy (LCOE)}

This is the ratio of the lifetime costs to the lifetime electric power generation, both of which are discounted back to a base year that reflects the average capital cost [103]. This metric is used to compare the cost of energy generated by a renewable technology with that of a traditional fossil fuel generating unit [105]. The LCOE is given as:

$$
\begin{gathered}
L C O E=\frac{T L C C}{\sum_{m=1}^{N}\left(E_{m} *(1+r)^{-} m\right)} \\
T L C C=\sum_{m=0}^{N} \frac{I C_{m}}{(1+r)^{m}}
\end{gathered}
$$

where TLCC is the total life cycle cost, $I C_{m}$ represents the investment cost in period $m$ and $E_{m}$ is the energy output or saved in year $m$.

In addition, the $L C O E$ which considers the current dollar value is known as the nominal $L C O E$ while the real $L C O E$ is a fixed dollar inflation-adjusted value. The nominal $L C O E$ uses a discount rate without taking into account inflation rate and the real $L C O E$ applies a discount rate with the inflation rate [105].

\subsubsection{Simple payback period}

The simple payback (SPB) period is another pivotal financial tool used to estimate the number of years it will take to recover the project cost of an investment made [105]. Also, it is important to note that the SPB does not consider the time value of money and with a zero discount rate. The SPB is given as the first point in time when the following expression is satisfied [105]: 


$$
\sum_{m} \Delta I I C_{m} \leq \sum_{m} \Delta S_{m}
$$

where $\Delta I I C_{m}$ represents the incremental investment costs at zero discount rate in period $m$ and $\Delta S_{m}$ is the sum total of the annual cash flows net annual costs at zero discount rate in period $m$.

The choice of a suitable measure in determining the economic value of DG deployments depends on various factors such as regulation, risk, financing, cash flow and investor's perspective. However, it is safer to evaluate these measures in order to have a more accurate assessment of the economic viability of a project [104], [105]. For instance, although the SPB measure gives a rapid sense of a project viability in a quick and simple fashion, it is however, not a recommended metric for DG installations investment requiring debt financing. This is because it ignores the discount rate, taxes and explicit consideration of project financing. However, in terms of project risk assessment, the SPB is a recommended and commonly used metric because of its capability in providing a quick evaluation of the period of time when the capital invested is at risk [104], [105].

Moreover, the NPV measure is a commonly used and recommended metric for accounting for all costs incurred by the society as a result of DG project investments such as social and environmental costs. Also, NPV can be very useful for selecting a DG project out of various mutually exclusive options. This is due to the fact that it explicitly evaluates the value of a DG project by giving larger investments greater propensity for potentially higher returns. However, one major draw back of the NPV metric is that it is highly impacted by discount rates. A marginal change in the discount rate will significantly affect the NPV value [104], [105].

The LCOE metric is recommended for ranking DG projects with limited budget because it provides a proper basis for ordering of alternatives until the investment budget is exhausted. It simply combines all present and future costs of investing in a DG project. However, the LCOE measure 
does not consider the variation in the sizes of investment when selecting a project among mutually exclusive options [104], [105].

\subsection{Results and discussion}

This section presents the performance analysis of the grid-connected 10 $\mathrm{kWp}(40 \times 250 \mathrm{Wp}) \mathrm{PV}$ system. We present results from our analysis, the National Renewable Energy Laboratory's (NREL) system advisory model (SAM) and Web Based PV System Monitoring and Reporting (WBPSMR). In order to monitor the behaviour of the PV system, it is pivotal to study the meteorological data recorded in the weather station covering the site location. Therefore, we have used full data set from National Institute of Water and Atmospheric research (NIWA) solarview tool [108] for our analysis.

Figure 3.3 shows the monthly averaged total in-plane irradiation and ambient temperature of the installation site. Significant peaks of in-plane insolation also known as Peak Sun Hour (PSH) occur during the summer period while the $\mathrm{PSH}$ dropped drastically during winter. Furthermore, the monthly average daily wind speed varied between $0.5 \mathrm{~m} / \mathrm{s}$ in most parts of the months and $21.6 \mathrm{~m} / \mathrm{s}$ in October. The relative humidity ranged from 38 to $97 \%$ and the maximum global irradiance was $1045 \mathrm{~W} / \mathrm{m}^{2}$. In addition, Figure 3.4 shows the module temperature as it varies across the entire year, with high temperatures during the summer period.

Analysis as depicted in Figure 3.5 shows an interesting pattern of the monthly capacity factors and performance ratios over the entire year. The monthly capacity factors increased proportionately with the in-plane solar irradiation with an annual average value of $12.5 \%$. The availability of a high solar resource as witnessed during summer period, led to increase in capacity factors (shown in Figure 3.5 ) and the final yield (shown in Figure 3.6).

However, high values of PSH does not necessarily translate to high 


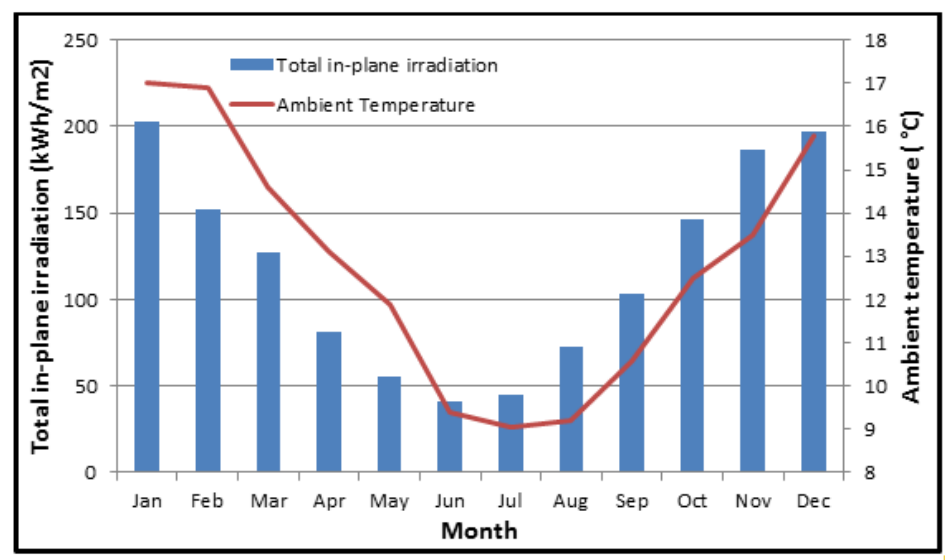

Figure 3.3: Monthly averaged total in-plane irradiation and ambient temperature

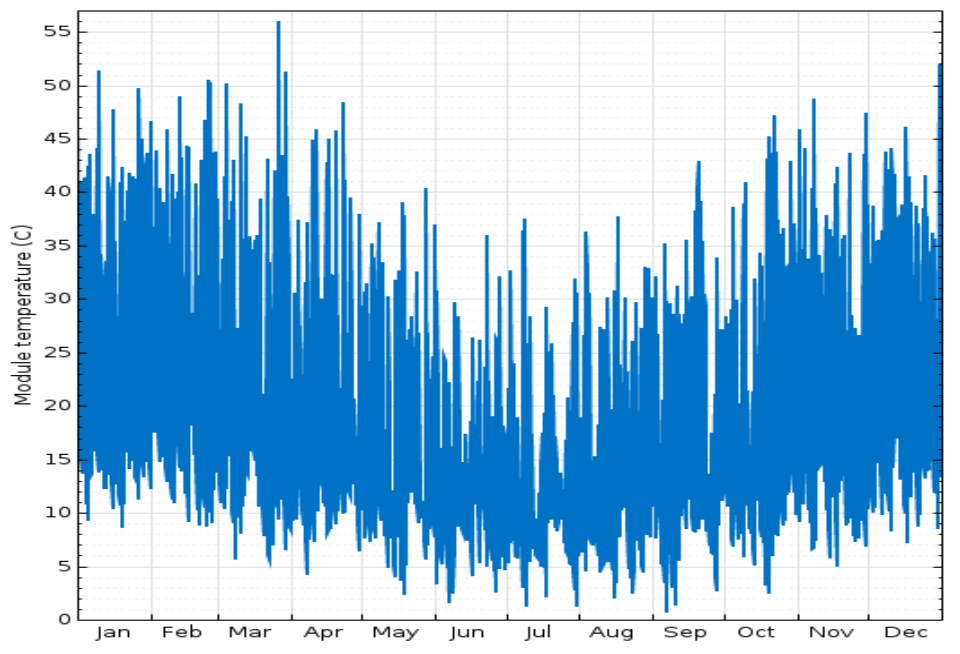

Figure 3.4: Annual module temperature 
performance ratios as shown in Figure 3.5. This is traceable to the impact of temperature losses on the overall performance of the PV array. As the temperature of the location increases, the $P R$ values also dropped accordingly. Therefore, $P R$ values are lower during summer period and higher in winter [75]. In addition, the system and array capture losses increased as the in-plane insolation increases.

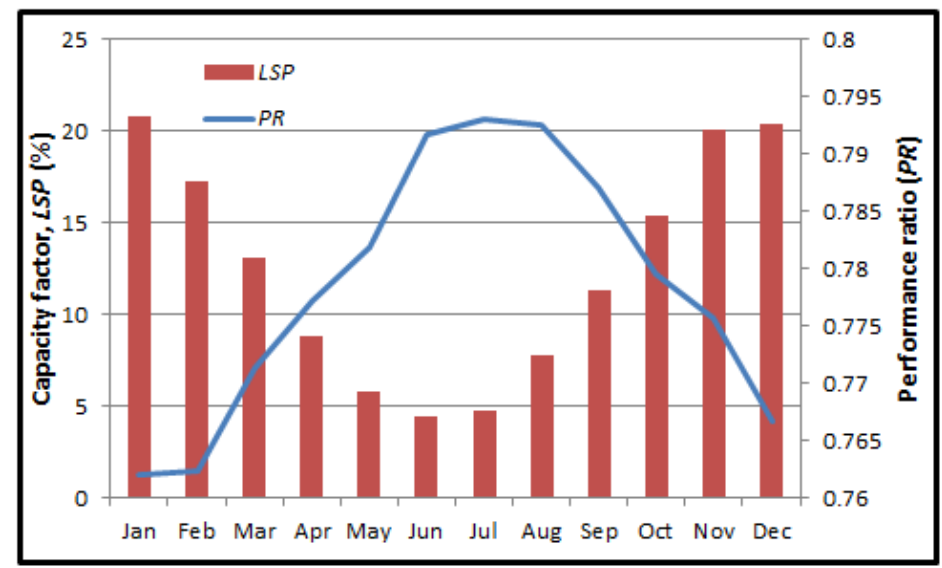

Figure 3.5: Monthly capacity factors and performance ratios of the PV array

Figure 3.6 depicts the monthly mean daily final yield, array capture and system losses. The monthly average daily array yield ranged from 1.2 $\mathrm{h} / \mathrm{d}$ (June) to $5.4 \mathrm{~h} / \mathrm{d}$ (January), while the final yield varied between 1.1 $\mathrm{h} / \mathrm{d}$ (June) to $4.9 \mathrm{~h} / \mathrm{d}$ (January) . The average annual final yield and reference yield were $2.99 \mathrm{~h} / \mathrm{d}$ and $3.87 \mathrm{~h} / \mathrm{d}$ respectively. The monthly averaged daily array capture losses ranged from 0.19 (June) to $1.14 \mathrm{~h} / \mathrm{d}$ (January) while the system losses varied from 0.09 (June) to $0.42 \mathrm{~h} / \mathrm{d}$ (January).

The PR of the considered PV array experienced a slight variation within the range of $76-79 \%$, and the annual average value was $78 \%$. This is comparable to the range of values (0.6-0.8) reported by IEA-PVPS Task 2 for grid-connected PV systems [76].

The monthly inverter efficiency ranged from $94.9 \%$ to $95.7 \%$ with higher 


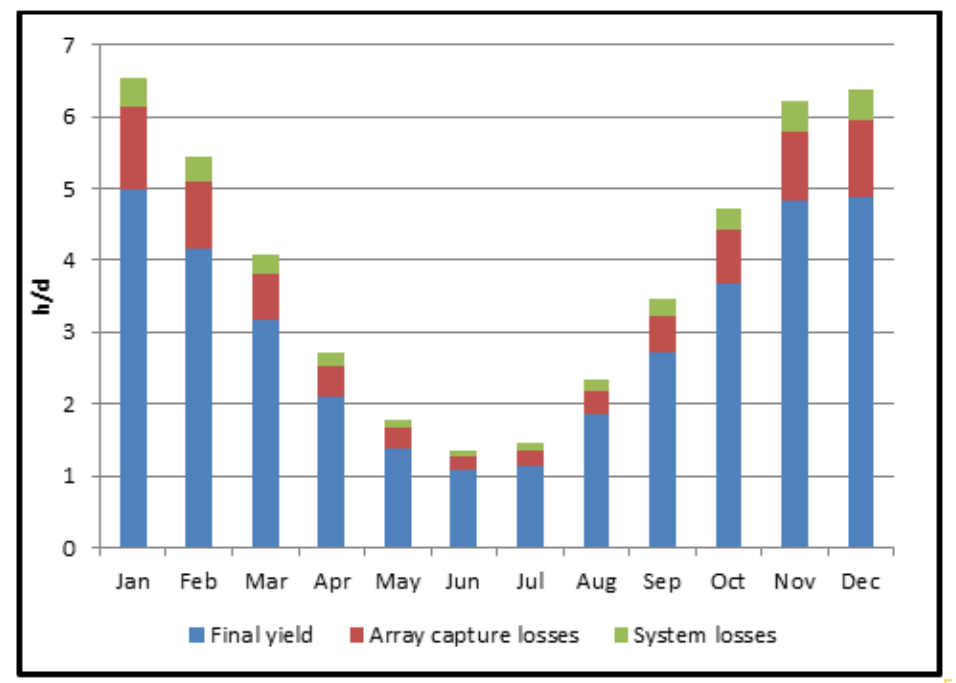

Figure 3.6: Monthly averaged daily final yield, PV array capture losses and system losses

values during the high PSH as shown in Figure 3.7. In addition, the monthly system efficiency ranged from $11.71 \%$ to $12.19 \%$ with higher values during low in-plane solar insolation as shown in Figure 3.7. The average annual system efficiency of the PV array is $11.96 \%$.

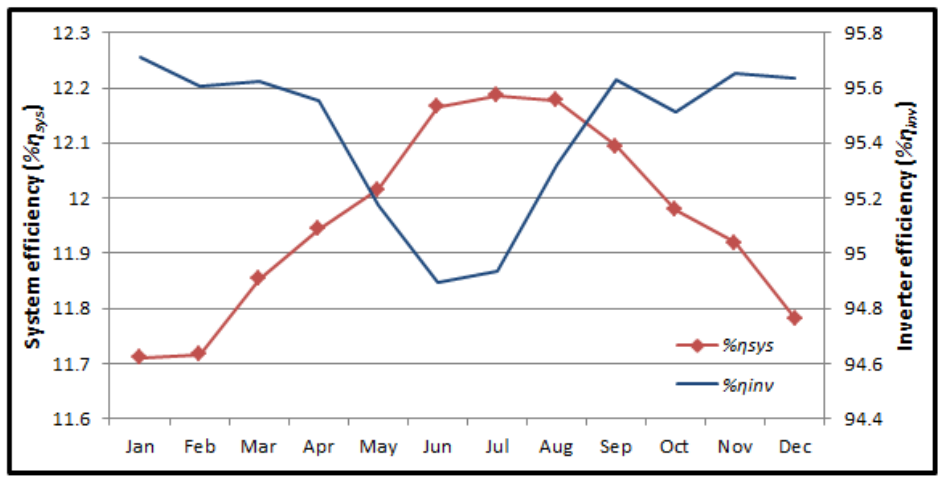

Figure 3.7: Monthly system and inverter efficiencies

In addition, the $10 \mathrm{kWp}$ PV array generated an average energy output of $1298 \mathrm{kWh}$ during the summer period ranging from $978 \mathrm{kWh}$ to 1546 
$\mathrm{kWh}$. During low PSH values in winter, the energy output ranged from $322.8 \mathrm{kWh}$ to $816.3 \mathrm{kWh}$. From our analysis, the annual average energy output was $910.13 \mathrm{kWh}$. However, from the WBPSMR, the average energy output was $1058.33 \mathrm{kWh}$, with the monthly energy output from the two 5 $\mathrm{kW}$ inverters shown in Figure 3.8. Low energy output in January was

Power Generated Per Month (kWh)

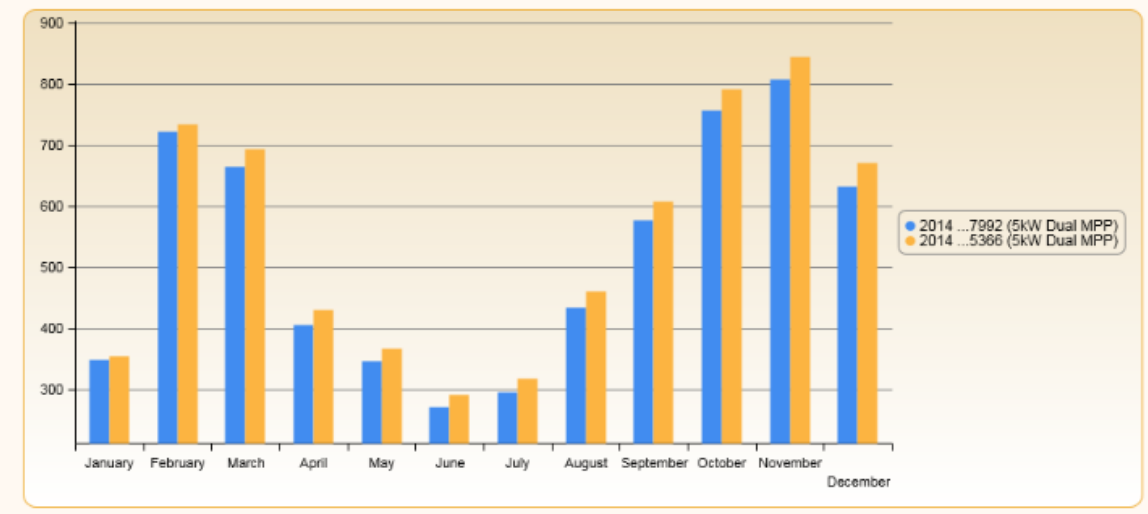

Figure 3.8: WBPSMR monthly PV output energy

because the site was commissioned at the middle of the month. The two strings of the PV array are mounted in an open space close to the school's football field, and the amount of dust collected over them varies, which causes variation in the array and inverter output as depicted in Figure 3.8. Also, the daily power generated is shown in Figure 3.9.

\subsection{Comparison of PV systems performance}

Performance analysis metrics allow cross-comparison between different PV systems operating under diverse climatic conditions [75]. Table 3.3 shows performance of various utility interactive PV systems across different locations.

The annual average final yield of the m-Si modules in this study is 

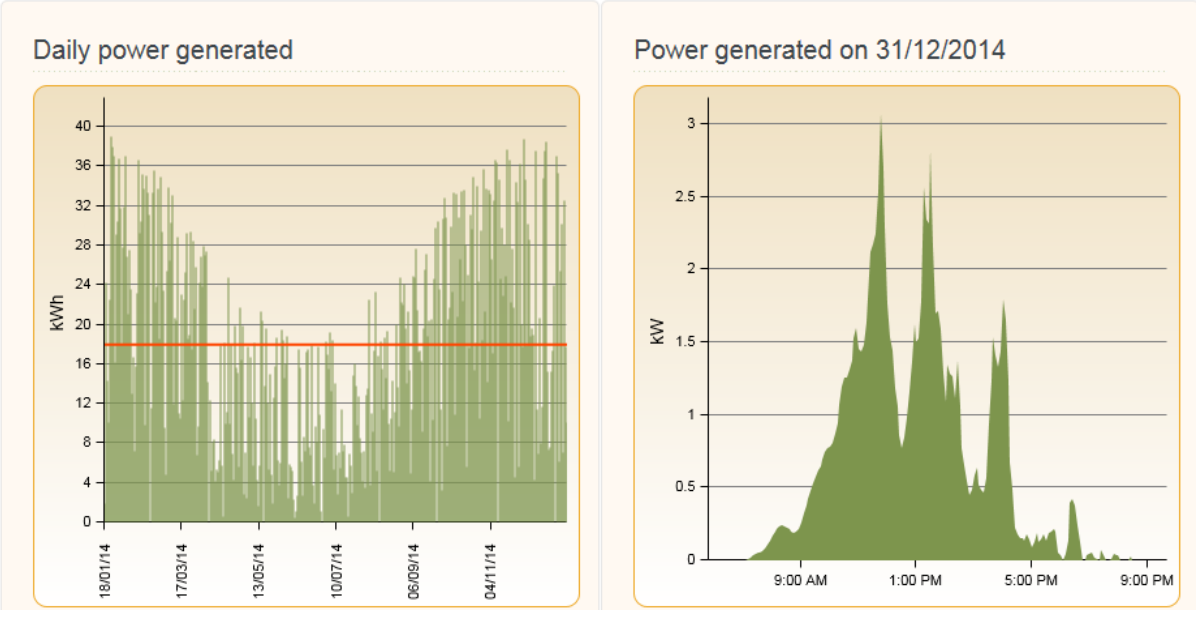

Figure 3.9: WBPSMR daily power generated

higher than the ones reported in Ireland and Northern Ireland. However, it is lower than the ones reported in Iran and India. Also, the annual average PR in this study falls within the range (0.25-0.9) reported by IEA PVPS Task 2 for 170 grid-connected PV systems in different countries of the world [76]. In addition, the PR for $\mathrm{m}-\mathrm{Si}$ technology is lower than that of $\mathrm{p}$-Si for most of locations with exception of Greece which ranged from 0.58 to 0.73 .

\subsection{Economic analysis}

The computation of the economic measures described is presented in this section using the NREL's SAM financial model. Although there is no feedin tariff legislation for the PV system in New Zealand, its economic viability especially for schools is shown in this chapter. A number of assumptions and inputs required in determining the financial metrics for the 10 kWp PV system are presented in Table 3.4. Also, the monthly energy consumption and peak demand are shown in Figure 3.10. The total project cost was NZD 28000 (USD 19600) which included the system, balance of 


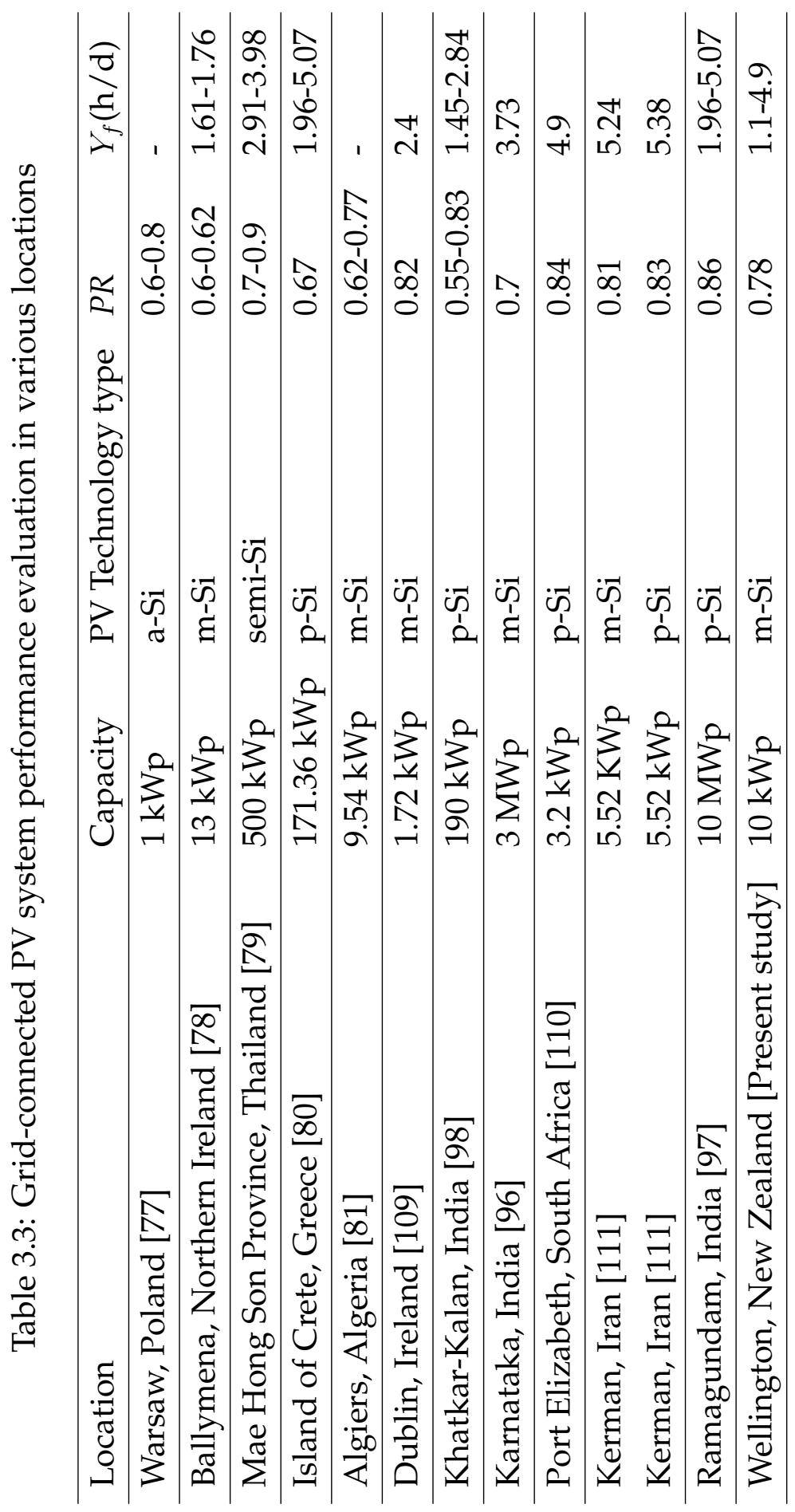


equipment (BOS), installation, installer margin and overhead costs. Therefore, the total installed cost per capacity was USD 1.96/Wdc.

Table 3.4: Inputs and assumptions used in the analysis

\begin{tabular}{ll}
\hline System size $(\mathrm{kWp})$ & 10 \\
\hline Operation and maintenance cost & \\
(Fixed cost by capacity) $(\$ / \mathrm{kW}-\mathrm{yr})$ & 20 \\
\hline System salvage value $(\$)$ & 0 \\
\hline Panel tilt (degrees) & $30^{0}$ \\
\hline Panel azimuth (degrees) & $0^{0}$ \\
\hline Annual panel degradation $(\% /$ year $)$ & $0.5 \%$ \\
\hline Inflation rate (\%/year) & $2.5 \%$ \\
\hline Real discount rate (\%/year) & $4 \%, 6 \%$ and $8 \%$ \\
\hline Analysis time period (years) & 25 \\
\hline Load profile used & school load profile \\
\hline Grid buyback rate (c/kWh) & 15 \\
\hline electricity retail rate (c/kWh) & 25 \\
\hline Corporate tax rate & $\mathrm{NA}$ \\
\hline
\end{tabular}

The cash flow is presented in Table 3.5 and results obtained are given in Table 3.6 with three discount rates. The discount rate has significant impacts on the NPV and $L C O E$, with $4 \%$ rate giving the highest $N P V$ and lowest norminal $L C O E$ values which makes it an appropriate rate for such a system. Figure 3.11 shows payback cash flow with a simple payback period of 6.4 years. In addition, the electricity to/from the grid across the entire year is shown in Figure 3.12. During periods of low insolation, significant amount of electricity was supplied from the grid, while excess 


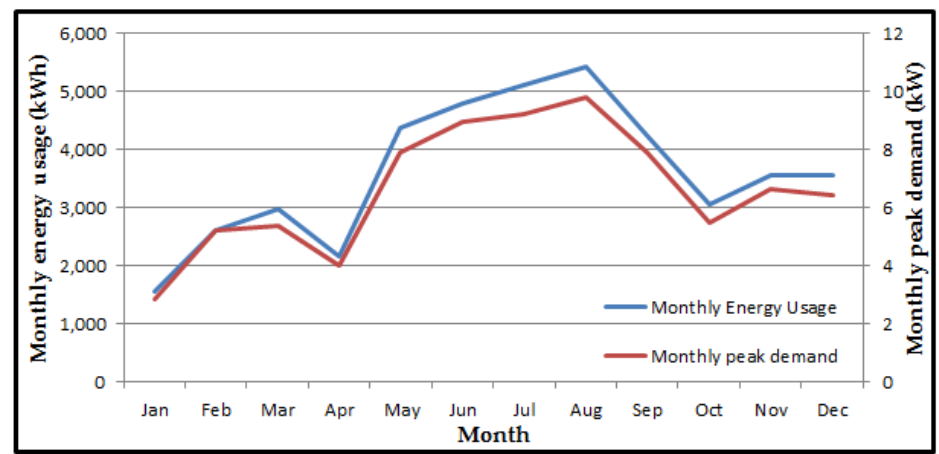

Figure 3.10: Monthly energy consumption and peak demand Table 3.5: Cash flow

\begin{tabular}{ll}
\hline Metric & Value \\
\hline Annual energy (year 1) & $16158 \mathrm{kWh}$ \\
\hline Energy yield (year 1) & $1616 \mathrm{kWh} / \mathrm{kW}$ \\
\hline Electricity bill without system (year 1) & $\$ 8310$ \\
\hline Electricity bill with system (year 1) & $\$ 5229$ \\
\hline Net savings with system (year 1) & $\$ 3082$ \\
\hline Net capital cost & $\$ 19600$ \\
\hline Equity & $\$ 19600$ \\
\hline Debt & $\$ 0$ \\
\hline
\end{tabular}

Table 3.6: Economic measures evaluation of the $10 \mathrm{kWp}$ system

\begin{tabular}{llll}
\hline Discount rate & $4 \%$ & $6 \%$ & $8 \%$ \\
\hline Real LCOE $(\mathrm{c} / \mathrm{kWh})$ & 9.43 & 11.19 & 13.09 \\
\hline Nominal LCOE $(\mathrm{c} / \mathrm{kWh})$ & 12.1 & 14.1 & 16.2 \\
\hline$N P V(\$)$ & 22000 & 14600 & 9100 \\
\hline
\end{tabular}




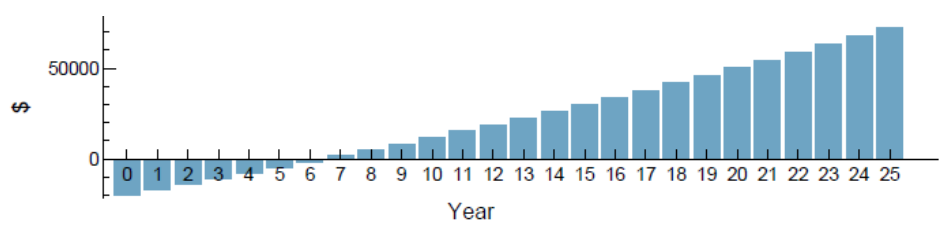

Figure 3.11: Payback cash flow (simple payback period $=6.4$ years)

power was exported to the grid during periods with high PSH and minimum load.
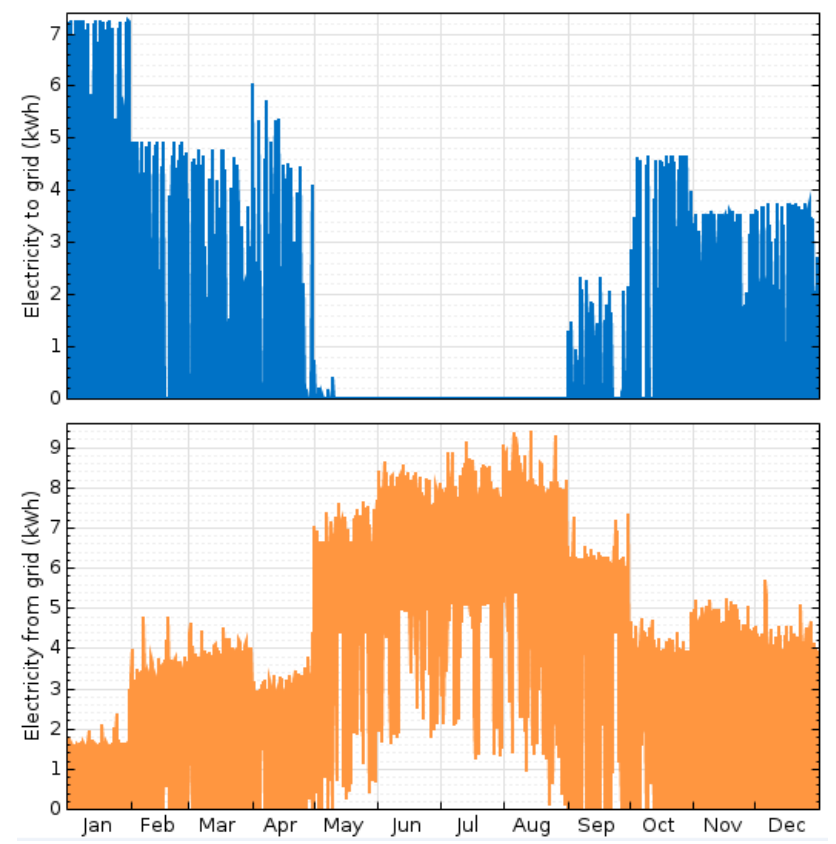

Figure 3.12: Annual electricity to/from the grid

\subsubsection{Actual economic analysis}

The actual financial analysis of the power consumption before and after the installation of the $10 \mathrm{kWp}$ PV system is presented in this section. The annual cost of the school's power consumption before the PV system integration was NZD $\$ 8380.86$. After the interconnection, the school's energy 
usage cost reduced to NZD \$3758.77.

The consumption pattern (obtained from the data logging system) of the school is shown in Figure 3.13 for pre- (without PV-2013) and postinstallation (with PV-2014) of the PV system. It shows a dramatic drop in $\mathrm{kWs}$ used and amount of money spent on power bills from the utility. In addition, as shown in Figure 3.13, the power consumed after the PV system installation represents the amount of power consumed minus the amount of excess power generated in a particular month. The excess power export to grid usually occurs at low load condition which happens during weekends and holidays. Also, the spike between November and December is due to readings carried over to the next month. Furthermore, from our analysis the total amount of power consumed and cost of power reduced significantly by $32 \%$ and $45 \%$ respectively at the end of the year 2014. In monetary terms, the school saved about NZD \$4700 in 2014 on power bills, which matches closely with the payback period given by the SAM's financial model.

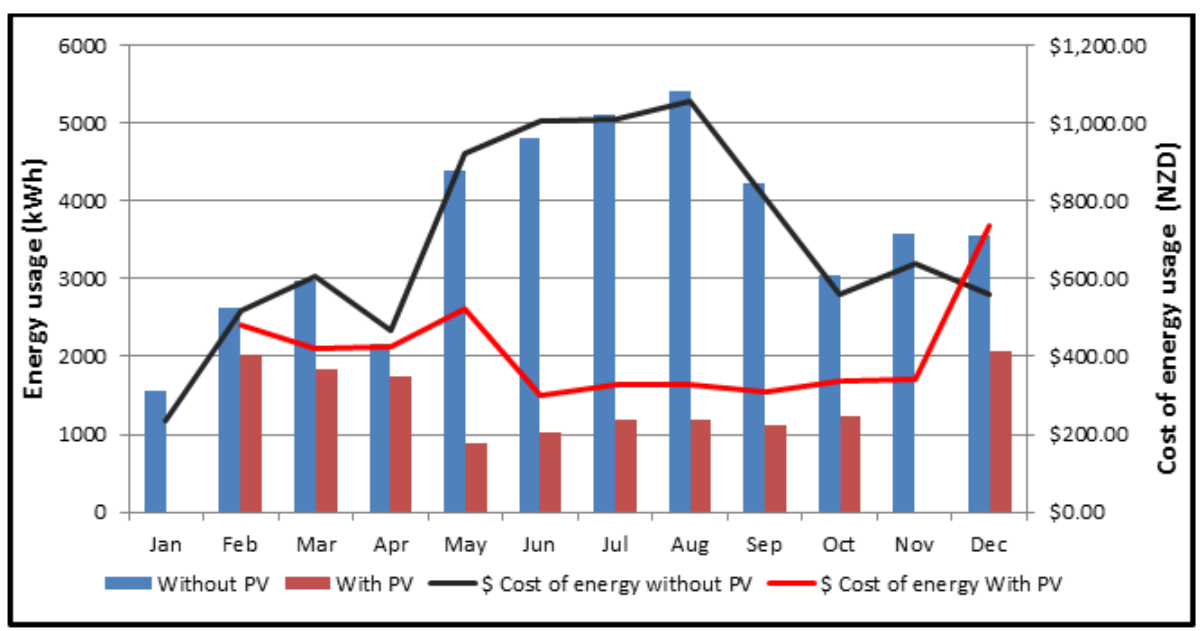

Figure 3.13: School's energy usage and cost of energy for pre- and postinstallation of the PV array 


\subsection{Discussion}

From the analysis, the main conclusions are given as follows:

- The final yield, $\left(Y_{f}\right)$, of the PV system ranged from 1.1 to $4.9 \mathrm{~h} / \mathrm{d}$, with an annual average value of $2.99 \mathrm{~h} / \mathrm{d}$. There is a direct proportional relationship between the peak sun hour (PSH) and $Y_{f}$. Also, the array capture and system losses increased with high values of PSH.

- The availability of a high solar resource led to increase in the capacity factor as witnessed during the summer months. The average annual capacity factor was $12.5 \%$.

- The performance ratio $(P R)$ experienced a slight variation within the range of $76-79 \%$, with an annual average value of $78 \%$. Temperature has a significant impact on the overall performance of the PV system. The maximum cell temperature was $52^{\circ}$ in the month of January and the minimum was $44.05^{\circ}$ in July.

- The annual power generation efficiency of the PV system was $11.96 \%$, which ranged from $11.71 \%$ to $12.19 \%$.

- During summer months, the average energy output was $1298 \mathrm{kWh}$, which ranged from $978 \mathrm{kWh}$ to $1546 \mathrm{kWh}$. However, the energy output during winter periods ranged from $322.8 \mathrm{kWh}$ to $816.3 \mathrm{kWh}$.

- In addition, from the financial evaluation at $4 \%, 6 \%$ and $8 \%$ discount rates, the levelized cost of energy are $12.1,14.1$ and $16.2 \mathrm{c} / \mathrm{kWh}$ respectively. Also, the net present value are USD 22000, 14600 and 9100 respectively with a simple payback period of 6.4 years.

- Over the monitored period (2014), the cost of grid power consumption reduced from NZD \$8380.86 to NZD \$3758.77, which resulted in savings of approximately NZD $\$ 4700$ which matches closely with 
the payback period given by the SAM's financial model. Overall, the total amount of grid power consumed reduced significantly by $32 \%$. The PV system saves the school up to half of its electricity bill during summer and a third in winter.

Grid-tied PV systems provide opportunity for their owners to have access to upstream energy market, contribute to network capacity and ancillary services.

\subsection{Summary}

The goal of this chapter is to evaluate performance metrics that can be used for cross-comparison between different PV systems in terms of design, technology and diverse climatic conditions. These metrics by extension is useful in the projection of the future value of grid-tied PV-DG which is very vital for effective system planning and operations.

Also, the evaluation of these parameters help to assess product quality, determine future needs and identify failures in system components. For instance, with the PR metric, system component malfunction such as inverter failure can be easily detected for utility-tied PV systems. In addition, other factors which could affect the PR include PV module soiling, shading and the ratio of the measured array efficiency to the nominal array efficiency. Properly maintained PV plants have a high tendency to operate optimally with high $P R$ value and availability.

\subsection{Next chapter}

In order to address grid related issues, the next chapter by extension focuses on the impact of such single-phase systems distributed on singlephase nodes of a distribution feeder. A typical school load demand and solar irradiance profile at a 5-minute resolution are incorporated in the test feeder. This PV system integration scenario is now very pertinent 
with many schools and small firms deploying single-phase PV-DG to offset power demand during peak sun hours. 


\section{Chapter 4}

\section{The impact of single-phase grid-connected distributed PV systems on the distribution network}

This chapter is an extension of the previous and provides a detailed active distribution power flow analysis with different DG generator models. In this chapter, an impact analysis of the utility-interactive single-phase PV systems distributed on all the single-phase load nodes of the traditional IEEE-13 bus distribution test feeder is presented. The normal current injection power flow algorithm implemented in OpenDSS has been used as the solution algorithm in this research. The PV-DG can be modeled as a constant $\mathrm{PQ}$ or $\mathrm{P}|\mathrm{V}|$ node with varied impacts in power flow studies for the unbalanced active distribution network. The results of this study have been peer-reviewed and published in [20]. 


\subsection{Introduction}

Single-phase PV-DG (i.e., $10 \mathrm{kWp}$ or less) injects power only on one phase of the distribution feeder and therefore, changes the voltage balance between the three-phase voltages [30]. This condition has the tendency of exacerbating the voltage unbalance in the distribution network, even with voltage values within the limits of ANSI C84.1-2006 [30], [33]. These integration issues have led to the adoption of standards such as ANSI C84.1 [30], IEC 61727 [112], IEEE-1547 [30] and UL 1741 [113] by utility companies as recommended practices to regulate the interconnection of PV-DG with electric power systems.

A number of studies on the impact of a single-phase PV-inverter system on the distribution have been carried out. Parmar et al. [114] investigated the impact of a single-phase grid connected photovoltaic installations with single-phase inverters on a low voltage network with hourly solar irradiance. Analysis carried out on IEEE 34 bus system showed that PV generators can be used to enhance voltage profile and unbalance along the feeder. Also, Shahnia et al. [56] presented a sensitivity analysis of voltage imbalance at various points on a distribution feeder with rooftop PV systems. Results presented confirmed that voltage unbalance increase or decrease is a function of the load on the phase where the PV system was installed, location and rating of the PV.

Awadallah et al. [115] demonstrated with a laboratory setup which employed a PV single-phase inverter and three-phase transformer in order to test the system performance under phase unbalance and harmonics. The experimental setup showed that voltage and current total harmonic distortion were within permissible limits, while losses and efficiency of the transformer increased with loading. El-Naggar et al. [116] proposed the use of a three-phase PV inverter to mitigate the voltage unbalance caused by single-phase PV systems in a low voltage network using a 10-minute solar irradiance normalised on its maximum peak. A total of 62 grid-tied 
PV plants were connected, out of which 21 were three-phase units and the rest single-phase systems. Results showed that maximum voltage unbalanced is reached during PV peak generation.

However, for interconnecting DG with the distribution network it is pivotal to take into consideration the type of generator models appropriate for power flow analysis in the unbalanced active distribution network. Based on control strategy, DG can be modelled as a P $|\mathrm{V}|$ (voltagecontrolled, grid-forming or constant power factor) node [117], [118] and a constant PQ (power-controlled) node [119], [120].

PQ nodes are modelled as negative load (fixed power injection) with current injection into the node and $\mathrm{P}|\mathrm{V}|$ bus treated also, as a fixed complex power element with a regulation scheme for maintaining the specified terminal voltage value within the reactive power limits [121], [122]. In addition, $\mathrm{P}|\mathrm{V}|$ node voltage magnitude is compensated for by obtaining the reactive current value for each $\mathrm{P}|\mathrm{V}|$ node which ensures this voltage value is the same as the scheduled value [123]. Also, for a DG operating in $\mathrm{PQ}$ mode, its positive-sequence representation is a constant power source and for $\mathrm{P}|\mathrm{V}|$ mode, it is assumed that the DG is equipped with voltage regulator and active power controller [124]. Further, for PQ buses, the complex power injected into the node is imposed and independent on the bus voltage [125].

Khushalani et al. [126] presented a three-phase unbalanced power analysis using $\mathrm{P}|\mathrm{V}|$ and $\mathrm{PQ}$ models for DG and the associated impacts of these models. Results presented showed that system losses are higher in $\mathrm{PQ}$ model than $\mathrm{P}|\mathrm{V}|$ model. Also, a $\mathrm{P}|\mathrm{V}|$ node reverts to a $\mathrm{PQ}$ node once the reactive power limit is exceeded which is defined by the power factor limits set between 0.8 and 1, leading or lagging. Hashemi et al. [127] also compared both models and confirmed that operating DG as $\mathrm{P}|\mathrm{V}|$ node decreased the voltage unbalance and total power losses. The result presented in [128] showed that $P|V|$ node enhanced the voltage profile and reduced active losses more than PQ node model. Maya et al. [129] confirmed in 
their study on the impact of distributed generation models that the $\mathrm{P}|\mathrm{V}|$ node model perform better than the PQ model in terms of voltage profile improvement and losses reduction.

The extant literature has generalised the operations of DG models for all types of DER in the distribution system. This study investigates the impact of these DG models for single-phase PV system integration with the grid. The contributions of this chapter are two folds:

- To analyse the impact of spatially distributed single-phase PV systems on the primary distribution network using a typical school load profile and solar irradiance data with a five-minute resolution to capture the effects of demand and solar PV variability.

- To compare the performance of $\mathrm{PQ}$ and $\mathrm{P}|\mathrm{V}|$ generator models in terms of the load buses voltage profiles and unbalance, feeder equipment loading, components of power losses and the computational cost required for a converged power flow solution.

\subsection{Load and solar PV modelling}

\subsubsection{Load modelling}

The distribution system loads are represented by the complex power consumed specified as the peak diversified demand [122]. The demand of the system considered in this study follows a typical normalized school load pattern with a peak demand during the mid-day. A five-minute resolution data provided in [130], which represents daily electrical energy usage in Wellington, New Zealand has been used for a typical school load profile. The load factor $(L F)$ is defined as the ratio of the area under the load demand curve in p.u. to the total period [131]. The $L F$ for this scenario is 0.748 . 


$$
L F=\sum_{t=1}^{24} \frac{p \cdot u \cdot \text { load demand }(\mathrm{t})}{24}
$$

Also, the time-varying and voltage dependent load model as given in [41], [132] is expressed as:

$$
\begin{gathered}
P_{k}(t)=P_{l k}(t) * V_{k}^{x_{p}}(t) \\
Q_{k}(t)=Q_{l k}(t) * V_{k}^{x_{q}}(t)
\end{gathered}
$$

where $P_{k}$ and $Q_{k}$ represent the active and reactive power injections respectively at bus k, $P_{l k}$ and $Q_{l k}$ are the active and reactive load at bus $k$ with reference to the nominal voltage, $V_{k}$ is the bus k voltage, $x_{p}$ and $x_{q}$ are the active and reactive load voltage exponents respectively . The values of $x_{p}$ and $x_{q}$ are given in Table 4.1 [132], [41].

Table 4.1: Load types and corresponding voltage exponents

\begin{tabular}{ccc}
\hline Load types & $x_{p}$ & $x_{q}$ \\
\hline Constant & 0 & 0 \\
Industrial & 0.18 & 6.00 \\
Residential & 0.92 & 4.04 \\
Commercial & 1.51 & 3.40 \\
\hline
\end{tabular}

\subsubsection{Solar PV renewable resource modelling}

\section{Solar irradiance modelling}

The inherent variability of solar PV is modelled using the Beta distribution probability density function (PDF) [41], [133], [134]. The irradiance data within the same hour over a number of days usually forms a bimodal probability density function bounded within finite limits. The PDF of solar irradiance, $g$, over a specified period is given as [41], [133], [134]: 


$$
f_{b}(g)= \begin{cases}\frac{\Gamma(\alpha+\beta)}{\Gamma(\alpha) \Gamma(\beta)} g^{\alpha-1}(1-g)^{\beta-1} & 0 \leq g \leq 1, \alpha, \beta \geq 0 \\ 0 & \text { otherwise }\end{cases}
$$

where $f_{b}(g)$ is the Beta distribution function of $g, g$ is the random variable of solar irradiance $\left(k W / m^{2}\right), \alpha$ and $\beta$ are shape parameters of the Beta distribution function which can be expressed in terms of the mean $(\mu)$ and standard deviation $\left(\sigma^{2}\right)$ of $g$ as follows:

$\beta=(1-\mu) *\left[\frac{\mu(1+\mu)}{\sigma^{2}}-1\right] ; \quad \alpha=\frac{\mu * \beta}{1-\mu}$

The PV module output power, $P_{P V}(g)$, at a given irradiance value is evaluated as [133]:

$$
P_{P V}(g)=V(g) I(g)
$$

Therefore, the total power output of the $\mathrm{PV}\left(P_{P V, t o t a l}\right)$ is given as:

$$
P_{P V, \text { total }}=\int_{0}^{1} P(g) f_{b}(g) d g
$$

Alternatively, $P_{P V}(g)$ can be expressed as [41], [135], [136]:

$$
P_{P V}(g)=N_{\text {modules }} * F F * V_{p v} * I_{p v}
$$

where:

$$
\begin{gathered}
F F=\frac{V_{m p p} * I_{m p p}}{V_{o c} * I_{s c}} ; \quad V_{p v}=V_{o c}-T_{v} * T_{\text {cell }} \\
I_{p v}=g\left[I_{s c}+T_{i} *\left(T_{\text {cell }}-25\right)\right] ; \quad T_{\text {cell }}=T_{\text {amb }}+g\left[\frac{N_{O T}-20}{0.8}\right]
\end{gathered}
$$




$$
\begin{aligned}
F F & =\text { fill factor } \\
I_{m p p} & =\text { current at maximum power point }(\mathrm{A}) \\
I_{s c} & =\text { short-circuit current }(\mathrm{A}) \\
N & =\text { Number of } \mathrm{PV} \text { modules } \\
T_{a m b} & =\text { ambient temperature }\left({ }^{0} \mathrm{C}\right) \\
T_{\text {cell }} & =\text { cell temperature }\left({ }^{0} \mathrm{C}\right) \\
T_{i} & =\text { current temperature coefficient }\left(A /{ }^{0} \mathrm{C}\right) \\
T_{v} & =\text { voltage temperature coefficient }\left(\mathrm{V} /{ }^{0} \mathrm{C}\right) \\
V_{m p p} & =\text { voltage at maximum power point }(\mathrm{V}) \\
V_{o c} & =\text { open-circuit voltage }(\mathrm{V})
\end{aligned}
$$

The expected PV output with respect to the irradiance data depicted in Figure 4.1 is calculated using (4.7) and shown in Figure 4.2. As expected, the maximum power output is $4.6 \mathrm{~kW}$ at noon time.

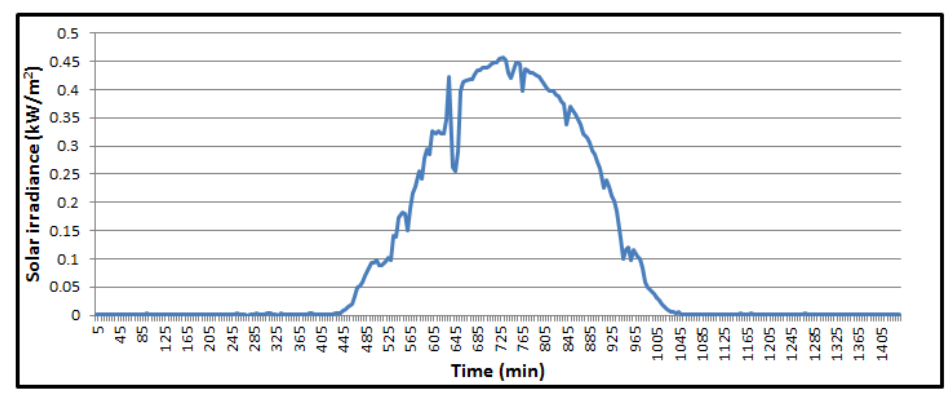

Figure 4.1: A typical daily solar irradiance with five-minute resolution during autumn in Wellington

\subsection{Distributed generation models}

In power flow studies, PV-DG can be modelled as PQ and $\mathrm{P}|\mathrm{V}|$ nodes depending on the type and utility interface used in connecting to the grid 


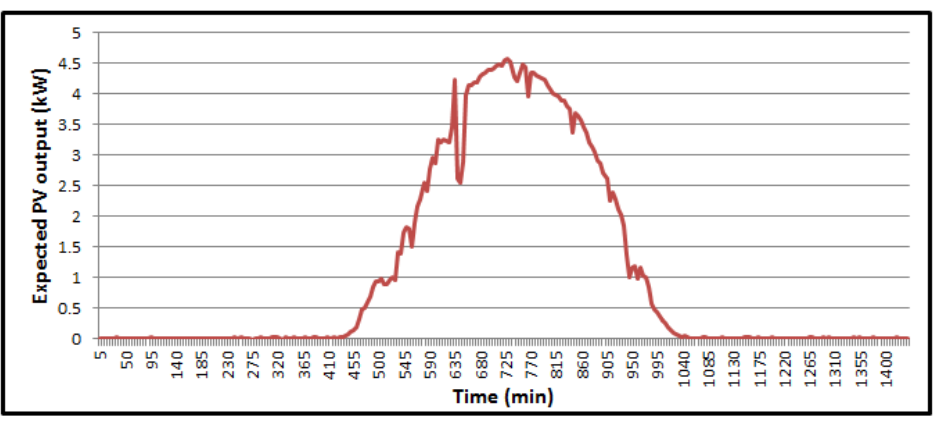

Figure 4.2: Expected daily PV array output

[129]. The PV-DG connection to the grid can be wye or delta with different bus voltages.

\subsubsection{Constant PQ node model}

For this type of node, the PQ injections from the PV-DG are represented as negative loads and the current injections for wye and delta connection are given by [122]:

$$
I_{L k}^{p h}=\left(\frac{S_{k}^{p h-n}}{V_{k}^{p h-n}}\right)^{*}
$$

where $I_{L k}^{p h}$ is the line currents for wye-connected load at node $k$.

$$
I_{L k}^{p h}=\left(\frac{S_{k}^{p h-p h}}{V_{k}^{p h-p h}}\right)^{*}
$$

where $I_{L k}^{p h}$ is the current magnitude for delta-connected load at node $k$. The positive-sequence representation of a PQ node represents a constant power source with a reactive power equal to one-third of the total threephase reactive power supplied to the grid by the PV-DG [124]. 


\subsection{2 $\quad \mathrm{P}|\mathrm{V}|$ bus model}

DG operating as a $\mathrm{P}|\mathrm{V}|$ node injects constant active power at a specified terminal voltage [123], [137]. However, an iterative procedure is required to maintain the specified voltage magnitude and monitor the DG's reactive power capability. The steps are outlined as follows [123], [126]:

1. At iteration $\tau$ and reactive power set to zero, the positive sequence voltage magnitude mismatch for all $\mathrm{P}|\mathrm{V}|$ nodes is given as:

$$
\Delta V_{k}^{\tau}=\left|V_{k}^{s c}\right|-\left|V_{k}^{\tau}\right| \leqslant \xi \quad \forall k \in \mathcal{S}:=\{1, \ldots, n\}
$$

where $\left|V_{k}^{s c}\right|$ is the scheduled or specified voltage magnitude for bus $k$. For possible mismatches exceeding the threshold, $\xi$, the next step is carried out.

2. Calculate the reactive current injection for $\mathrm{P}|\mathrm{V}|$ node by the following expression:

$$
Z_{k S} * I_{k Q}^{\tau}=\Delta V_{k}^{\tau}
$$

where $Z_{k S}$ is a positive sequence constant real impedance matrix known as the $\mathrm{P}|\mathrm{V}|$ node sensitivity matrix, with dimension same as the number of $\mathrm{P}|\mathrm{V}|$ nodes. $I_{k Q}^{\tau}$ is the positive sequence reactive current injection which gives a linear approximation necessary to eliminate the voltage magnitude mismatch. The reactive current injection at bus $k$ is therefore given as:

$$
\begin{aligned}
I_{k Q a}^{\tau} & =\left|I_{k Q}\right|^{\tau} * e^{j\left(90^{0}+\delta v_{k a}\right)} \\
I_{k Q b}^{\tau} & =\left|I_{k Q}\right|^{\tau} * e^{j\left(90^{0}+\delta v_{k b}\right)} \\
I_{k Q c}^{\tau} & =\left|I_{k Q}\right|^{\tau} * e^{j\left(90^{0}+\delta v_{k c}\right)}
\end{aligned}
$$

where $\delta v_{k a}, \delta v_{k b}$, and $\delta v_{k c}$ are voltage angles of the converged three phases of the $\mathrm{P}|\mathrm{V}|$ bus at node $k$. 
3. Since a constraint is applied to the amount of reactive power supply from the DG, it is important to calculate this amount using the $P|V|$ node voltage and the new current injection using this equation:

$Q_{k G}^{\prime \tau}=\operatorname{imaginary}\left(V_{k a} I_{k a}^{\prime *}\right)^{\tau}+\operatorname{imaginary}\left(V_{k b} I_{k b}^{\prime *}\right)^{\tau}+\operatorname{imaginary}\left(V_{k c} I_{k c}^{\prime *}\right)^{\tau}$

At the $\mathrm{P}|\mathrm{V}|$ bus $k$, the new current injection is the addition of the required reactive current and load current injections given as:

$$
\begin{aligned}
& I_{k a}^{\prime \tau}=I_{k Q a}^{\tau}+I_{k a}^{\tau} \\
& I_{k b}^{\prime \tau}=I_{k Q b}^{\tau}+I_{k b}^{\tau} \\
& I_{k c}^{\prime \tau}=I_{k Q c}^{\tau}+I_{k c}^{\tau}
\end{aligned}
$$

For each phase, the total reactive current the DG can inject into the grid before exceeding its limit is calculated by:

$$
I_{k Q}=\frac{Q_{k G} / 3}{\left|V_{k}\right|}
$$

where $Q_{k G}$ is the total three-phase reactive power injected by the DG unit at node $k$.

4. The amount of $Q_{k G}$ is then compared with limits specified using this expression: $Q_{k G}^{\min }<Q_{k G}^{\tau}<Q_{k G}^{\max }$

Within the given constraint, the DG is not permitted to exceed the maximum reactive power specified, and outside these limits the DG reverts to a PQ node. The above steps are repeated until the voltage magnitude of all the $\mathrm{P}|\mathrm{V}|$ nodes is less than the tolerance value. 


\subsection{Problem formulation}

The penetration of a distributed single-phase PV systems in the distribution network has varied impacts depending on the interconnection model, size and location of the PV-DG on the network. A very common adverse effect of large numbers of such PV-DG is increase in unbalance in the network, with random deployments over the three phases [33], [30]. The probability that $N$ units of these systems are all in the same phase is given as [33]:

$$
\operatorname{Pr}(N)=3^{(1-N)}
$$

Therefore, it as apparent from (4.16), that as the number of units increases, the probability of being on the same phase also decreases. For instance, if $N$ is set to values of 4,8 and 12, the probability that these units will be on the same phase equals to $3.7 \%, 0.046 \%$ and $0.00056 \%$ respectively. Generally, this leads to an unequal distribution of these singlephase units over the three phases and therefore, the system unbalance remains. However, with small sized PV-DG, overload and power losses (such as active load, reactive load and line losses) decrease, due to load current reductions. Also, the penetration of PV-DG within the hosting capacity of the feeder will offset active power flow, which invariably enhances the voltage stability [33].

\subsubsection{PV-DG impacts on the distribution network}

\section{Equipment overloading and Power losses}

Equipment overload and power losses on the distribution network depend on the rms value of the current [33]. The proliferation of PV-DG now brings generation closer to the end-use points which reduces the risk of high voltage levels and losses due to distance commonly experienced in the traditional network.The PV-DG can be used to offset the magnitude of the load current flow in a distribution grid as a current injection source, 
which eventually reduces the overall feeder loading [54].

The total power loss without PV-DG over all the feeder sections integrated over time, $S_{\text {loss }, W O P V}$, assuming a constant value of resistance per unit length across the entire feeder, can be expressed as [33]:

$$
S_{\text {loss }, W O P V}=\sum_{j=1}^{N} \int_{0}^{T}\left[I_{j}(t)\right]^{2} d t
$$

However, with PV-DG present, total power loss, $S_{l o s s, W P V}$, becomes:

$$
S_{\text {loss }, W P V}=\sum_{j=1}^{N} \int_{0}^{T}\left[I_{j}(t)-I_{j, P V}(t)\right]^{2} d t
$$

Subtracting (4.18) from (4.17) leads to power losses reduction, given as:

$$
\Delta S_{\text {loss }}=\sum_{j=1}^{N} \int_{0}^{T} I_{j, P V}(t)\left[2 I_{j}(t)-I_{j, P V}(t)\right] d t
$$

where $N, I_{j}, I_{j, P V}$ and $\Delta S_{\text {loss }}$ respectively represent the number of branches, branch " $j$ " current magnitude, PV-DG current injection at branch " $j$ " and power loss reduction.

Therefore, due to current reductions at penetration levels within the hosting capacity of the feeder, PV-DG can be used to relieve the capacity of the power deliver system (equipment) and reduce power losses through current injections.

In addition, total system losses can be calculated as follows [138], [139]:

$$
P_{L}+j Q_{L}=\sum_{k=1}^{N} V_{k} I_{k}^{*}
$$

The power loss of a line section connecting buses $j$ and $j+1$ without PVDG is expressed as:

$$
P_{L}(j, j+1)=\left[\frac{P_{j}^{2}+Q_{j}^{2}}{\left|V_{j}\right|^{2}}\right] * R_{j}
$$


The total active power loss without PV-DG, $P_{T, L}$, of the entire line sections of the feeder is expressed as:

$$
P_{T, L}=\sum_{j=1}^{N}\left[\frac{P_{j}^{2}+Q_{j}^{2}}{\left|V_{j}\right|^{2}}\right] * R_{j}
$$

However, with PV-DG connected at bus $j+1$ (represented as $m$ ), the active and reactive power flow from the source to this bus reduces as a result of the active and reactive power injections at the bus, while the power flows in other sections remains the same. Therefore, the basecase active power loss equation (4.22) with PV-DG installation is now rewritten as [41], [53]:

$$
\begin{array}{r}
P_{P V, L}=\sum_{j=1}^{m}\left[\frac{\left(P_{j}-P_{p v, m}\right)^{2}}{\left|V_{j}\right|^{2}}\right] * R_{j}+\sum_{j=m+1}^{N}\left[\frac{P_{j}^{2}}{\left|V_{j}\right|^{2}}\right] * R_{j}+\sum_{j=1}^{m}\left[\frac{\left(Q_{j}-Q_{p v, m}\right)^{2}}{\left|V_{j}\right|^{2}}\right] \\
* R_{j}+\sum_{j=m+1}^{N}\left[\frac{Q_{j}^{2}}{\left|V_{j}\right|^{2}}\right] * R_{j}
\end{array}
$$

Substituting (2.10) and (4.22) into (4.23), $P_{P V, L}$ becomes:

$$
\begin{gathered}
P_{P V, L}=\sum_{j=1}^{m}\left[\frac{P_{p v, m}^{2}-2 P_{j} P_{p v, m}}{\left|V_{j}\right|^{2}}\right] * R_{j}+\sum_{j=1}^{m}\left[\frac{\left.\alpha_{m}^{2} P_{p v, m}^{2}-2 Q_{j} \alpha_{m} P_{p v, m}\right]}{\left|V_{j}\right|^{2}}\right] * R_{j} \\
+\sum_{j=1}^{N}\left[\frac{P_{j}^{2}+Q_{j}^{2}}{\left|V_{j}\right|^{2}}\right] * R_{j} \\
P_{P V, L}=\sum_{j=1}^{m}\left[\frac{P_{p v, m}^{2}-2 P_{j} P_{p v, m}}{\left|V_{j}\right|^{2}}\right] * R_{j}+\sum_{j=1}^{m}\left[\frac{\alpha_{m}^{2} P_{p v, m}^{2}-2 Q_{j} \alpha_{m} P_{p v, m}}{\left|V_{j}\right|^{2}}\right] * R_{j}+P_{T, L}
\end{gathered}
$$


The net real power loss reduction, $\Delta P_{L}$, is the power loss difference between pre- and post-PV-DG installation.

$$
\Delta P_{L}=\sum_{j=1}^{m}\left[\frac{P_{p v, m}^{2}-2 P_{j} P_{p v, m}}{\left|V_{j}\right|^{2}}\right] * R_{j}+\sum_{j=1}^{m}\left[\frac{\alpha_{m}^{2} P_{p v, m}^{2}-2 Q_{j} \alpha_{m} P_{p v, m}}{\left|V_{j}\right|^{2}}\right] * R_{j}
$$

For positive value of $\Delta P_{L}$, it indicates that the active power loss is reduced with PV-DG integration, while negative $\Delta P_{L}$ implies active power loss increases with PV-DG. Also, the total basecase reactive power loss across the all feeder sections is given as:

$$
Q_{T, L}=\sum_{j=1}^{N}\left[\frac{P_{j}^{2}+Q_{j}^{2}}{\left|V_{j}\right|^{2}}\right] * X_{j}
$$

Similarly, with reference to (4.27), the total reactive power loss with with PV-DG installation is expressed as:

$Q_{P V, L}=\sum_{j=1}^{m}\left[\frac{P_{p v, m}^{2}-2 P_{j} P_{p v, m}}{\left|V_{j}\right|^{2}}\right] * X_{j}+\sum_{j=1}^{m}\left[\frac{\alpha_{m}^{2} P_{p v, m}^{2}-2 Q_{j} \alpha_{m} P_{p v, m}}{\left|V_{j}\right|^{2}}\right] * X_{j}+Q_{T, L}$

Also, the net reactive power loss reduction, $\Delta Q_{L}$, is the reactive power loss difference between pre- and post-PV-DG installation.

$$
\Delta Q_{L}=\sum_{j=1}^{m}\left[\frac{P_{p v, m}^{2}-2 P_{j} P_{p v, m}}{\left|V_{j}\right|^{2}}\right] * X_{j}+\sum_{j=1}^{m}\left[\frac{\alpha_{m}^{2} P_{p v, m}^{2}-2 Q_{j} \alpha_{m} P_{p v, m}}{\left|V_{j}\right|^{2}}\right] * X_{j}
$$

where $P_{j}$ and $Q_{j}$ represent active and reactive power injections at the $j$ th bus respectively, $P_{p v, m}$ and $Q_{p v, m}$ are PV-DG active and reactive power injections at the $(j+1)$ th bus respectively and $\left|V_{j}\right|$ and $N$ represent voltage magnitude at the bus $j$ and number of buses respectively. A positive value of $\Delta Q_{L}$, indicates that the reactive power loss is reduced with PV-DG integration, while negative $\Delta Q_{L}$ implies reactive power loss increases with PV-DG. 


\subsubsection{Voltage unbalance}

The penetration of distributed single-phase PV-DG in the unbalanced active distribution network can increase the existing unbalance by injecting power only on one phase of the feeder [30]. IEEE Standard Test Procedure for Polyphase Induction Motors and Generators defines Voltage unbalance, $V_{u n b}$ as [140]:

$$
V_{u n b}=\frac{\text { max. voltage deviation from the average voltage }}{\text { average voltage }} * 100
$$

Moreover, even without possible violation of the ANSI C84.1-2006 limits, high $V_{u n b}$ can still exist [30]. The random distribution of these units results in negative-sequence current, which increases the unbalanced negativesequence voltage [33]. At unacceptable high values (2.5 - 3\%), voltage unbalance can cause three-phase equipments such as the large chiller compressor to overheat and eventually stalls its operation [30]. Furthermore, depending on the location and size of the integrated PV-DG, the $V_{u n b}$ can either increase or decrease [56]. Also, $V_{u n b}$ tends to be more severe at the end of the feeder than at the beginning, which posses a serious operational issue for three-phase motors and other multi-phase utilization devices [30], [56], [59].

\subsection{Case study}

In this study, the IEEE-13 distribution test feeder is used to investigate the impact of distributed single-phase PV systems as shown in Figure 4.3. The test bus is a relatively highly loaded feeder with a peak demand of $3466 \mathrm{~kW}$ and $2120 \mathrm{kVAr}$ [141], following the normalized load curve as shown in Figure 4.4. Normalized five-minute irradiance data (shown in Figure 4.5) and school load profile are incorporated into the 13-bus model to investigate the associated impacts. The operating voltage constraint is 
between 0.95 and 1.05 p.u [30]. $10 \mathrm{kWp}$ solar PV systems (modelled as negative loads) are connected to 14 single-phase load nodes of the basecase network model through single-phase power converters operating at 0.985 power factor. The converters are oversized by $10 \%$ to ensure maximum real power from the PV array while supplying reactive power [57]. Also, the load buses connecting the PV systems are modelled as PQ and $\mathrm{P}|\mathrm{V}|$ buses. The values of the active power for each load bus are given in Table 4.2.

The penetration level, $P_{L}$, of the $P V$ systems within the distribution network is defined as [54], [142]:

$$
P_{L}=\frac{\sum_{k=1}^{n} P_{k, P V}}{P_{f d r, p e a k}}
$$

where $n$ is the total number of $P V$ units on the feeder, $P_{k, P V}$ represents the power rating (DC) of kth $P V$ unit connected to the feeder and $P_{f d r, p e a k}$ is the peak active load on the feeder.

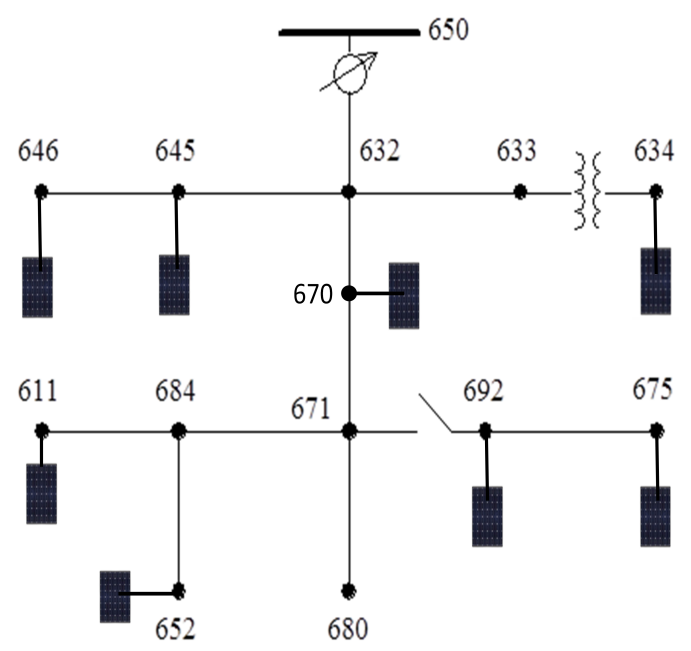

Figure 4.3: The modified IEEE-13 bus test network with single-phase PV 


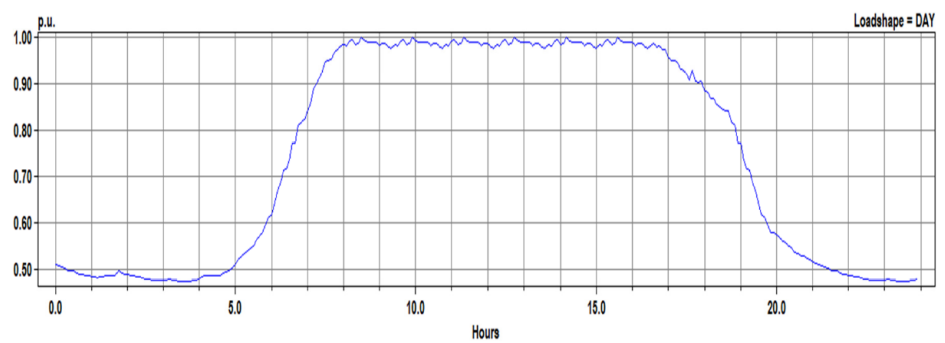

Figure 4.4: Normalized load profile of a school

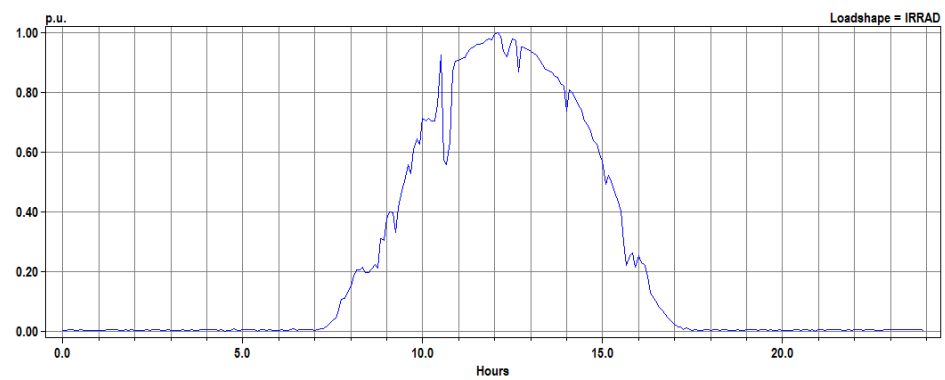

Figure 4.5: Normalized daily irradiance with five-minute resolution

Table 4.2: Single-phase load buses of IEEE-13 test network

\begin{tabular}{cccccc}
\hline Bus & Load $(\mathrm{kW})$ & Connection & Bus & Load $(\mathrm{kW})$ & Connection \\
\hline $634 \mathrm{a}$ & 160 & Wye & $675 \mathrm{a}$ & 485 & Wye \\
$634 \mathrm{~b}$ & 120 & Wye & $675 \mathrm{~b}$ & 68 & Wye \\
$634 \mathrm{c}$ & 120 & Wye & $675 \mathrm{c}$ & 290 & Wye \\
645 & 170 & Wye & 611 & 170 & Wye \\
646 & 230 & Delta & 652 & 128 & Wye \\
692 & 170 & Delta & $670 \mathrm{a}$ & 17 & Wye \\
$670 \mathrm{c}$ & 117 & Wye & $670 \mathrm{~b}$ & 66 & Wye \\
\hline
\end{tabular}

\subsection{Simulation results and discussion}

A 24-hour time series power flow is performed in Open-source distribution system simulator (OpenDSS) with varying demands and distributed single-phase PV-DG systems on the IEEE-13 bus test network. 


\subsubsection{Solar generation}

The resulting generation from the PV-DG installed at the single-phase load buses listed in Table 4.2 are shown in Figure 4.6. The profiles (for buses 645 and 670) show solar PV generation as negative load, which represent a typical generation profile at the listed load buses.

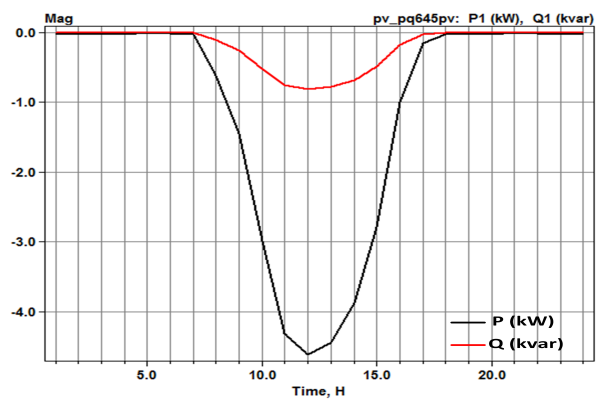

(a) PQ injections at bus 645

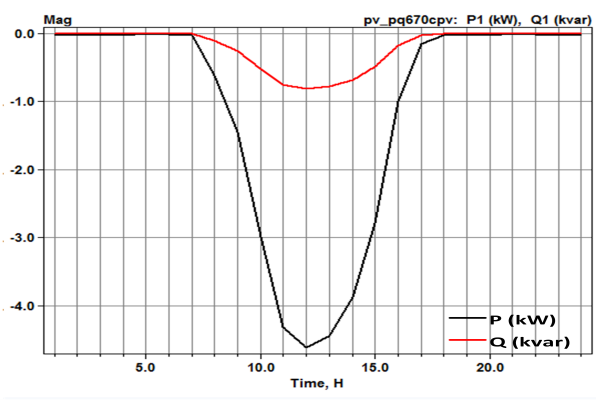

(b) PQ injections at bus 670

Figure 4.6: A typical active and reactive power injections at all singlephase load buses

\subsubsection{The impact of distributed single-phase PV systems}

The impacts of solar generation profiles spatially distributed in the distribution network are presented as follows:

\section{Voltage impact}

The PV-DG has a more direct impact on the voltage under different loading conditions of the distribution feeder phases. Figure 4.7 shows the voltage profile across the entire feeder with the blue and black phases being the heavily phases, and the red phase is the lightly loaded phase.

The branches in the profile represent power flow from one bus to another as depicted by the green line on the black phase. As expected of a 


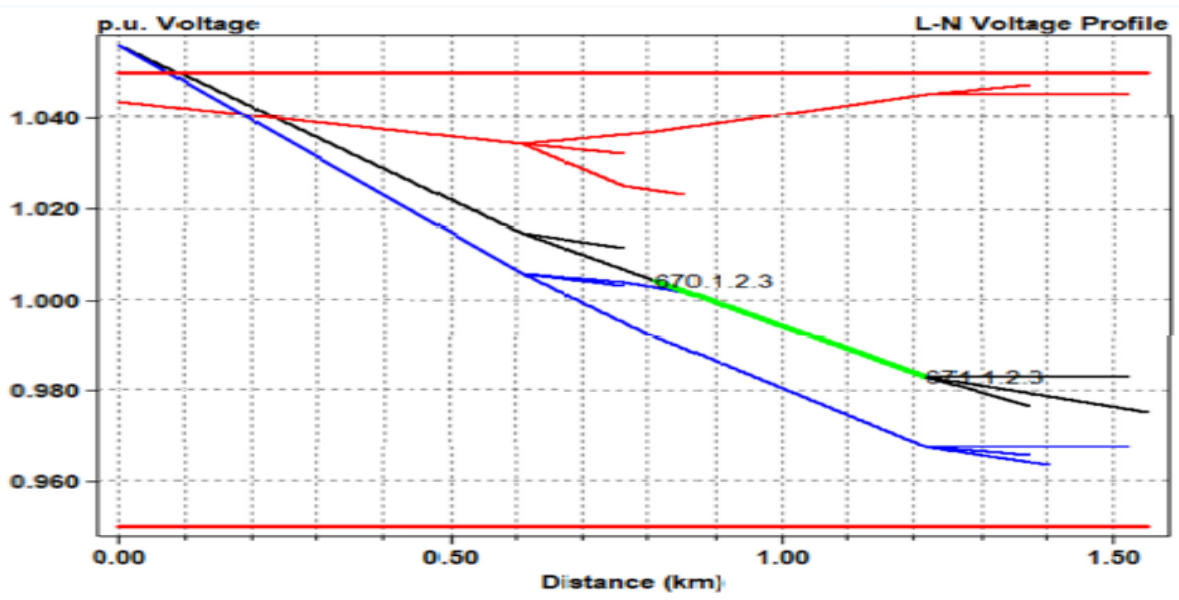

Figure 4.7: Voltage profile along the feeder

radial feeder, there is a voltage drop along the feeder as the distance from the substation increases.

The voltage profile for buses on the heavily loaded black phase is shown in Figure 4.8. These profiles show an interesting characteristics of the PQ and $\mathrm{P}|\mathrm{V}|$ models. At peak period when most loads are turned on (7 - 10:30 am), the PQ model drops the voltage, while there is a consistent increase in voltage profile in the case of the $\mathrm{P}|\mathrm{V}|$ model (this model assumes that DG to be equipped with a voltage regulator and active power controller [117], [118]) during hours of insolation.

Also, for the lightly loaded red phase, the voltage profiles of the buses on this phase are shown in Figure 4.9. In this case, the PQ model enhanced the profile slightly while $\mathrm{P}|\mathrm{V}|$ model increased the voltage profile significantly during the hours of insolation.

\section{Voltage unbalance}

Voltage unbalance impact is one of the limiting factors in the deployment of single-phase PV-DG in the unbalanced active distribution network. Singlephase PV-DG can either increase or decrease voltage unbalance depending on its size and location. A striking feature of the models during peak de- 


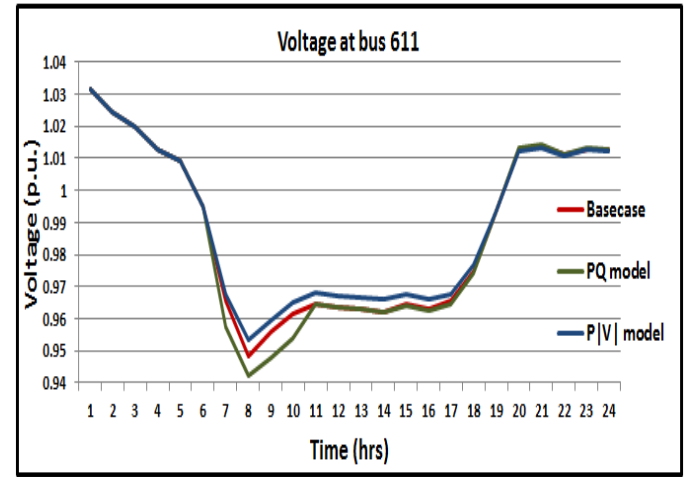

(a) Voltage profile at bus 611

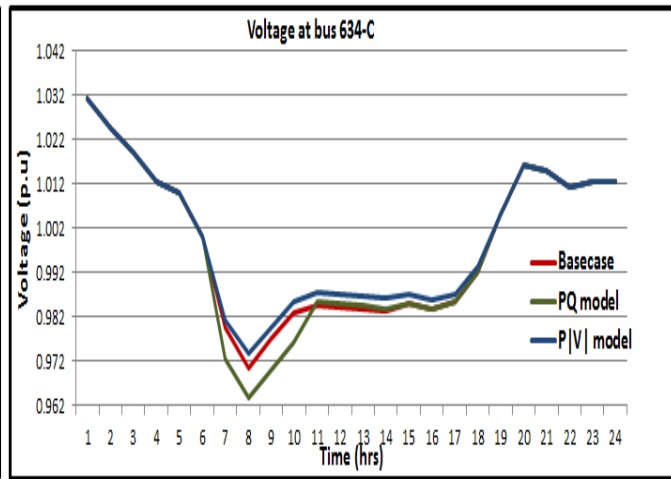

(b) Voltage profile at bus 634

Figure 4.8: Voltage profiles of buses on the heavily loaded black phase

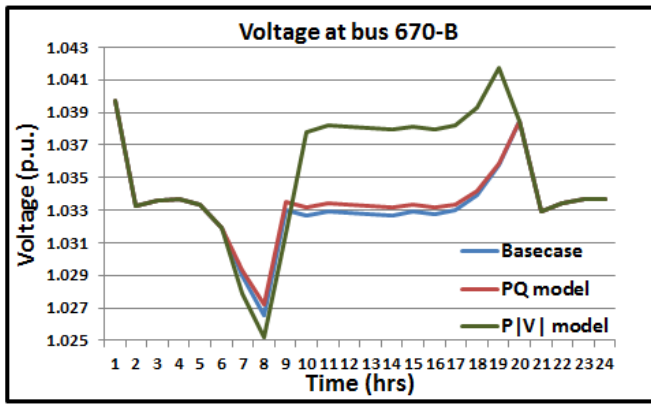

(a) Voltage profile at bus 670

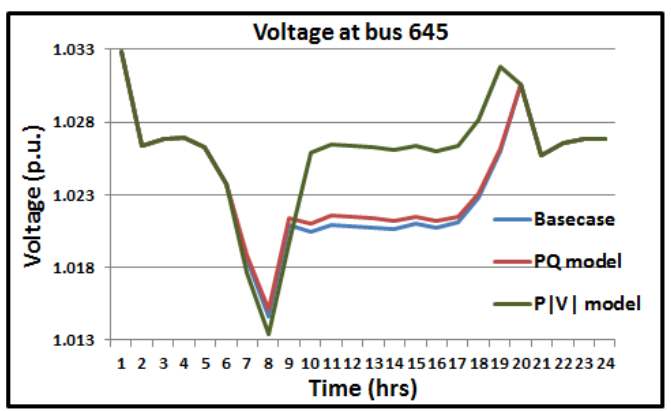

(c) Voltage profile at bus 645

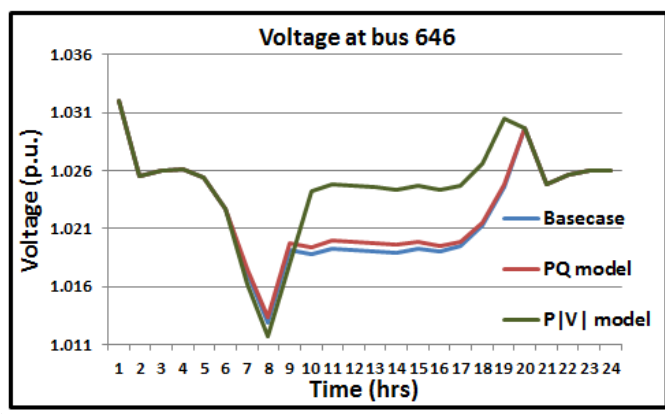

(b) Voltage profile at bus 646

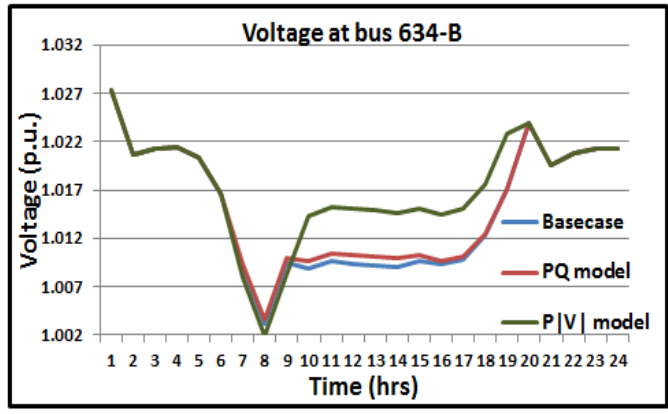

(d) Voltage profile at bus 634

Figure 4.9: Voltage profiles of buses on the red phase 
mand between 7 to 10:30 am is shown in Figure 4.10. At this time, the PQ model increases the unbalance and then decreases gradually, while $\mathrm{P}|\mathrm{V}|$ model reduced the unbalance during this period and then maintained a constant value above the basecase. Generally, high value of voltage unbal-

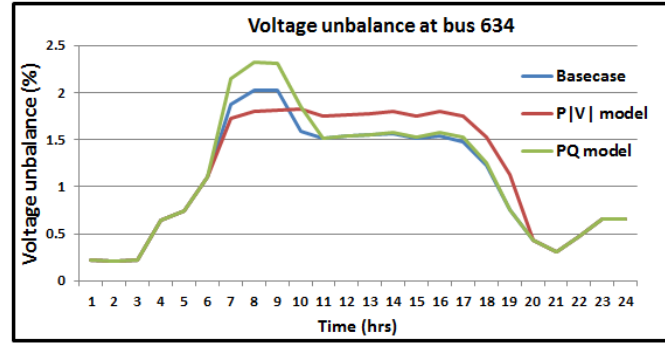

(a) Voltage unbalance at bus 634

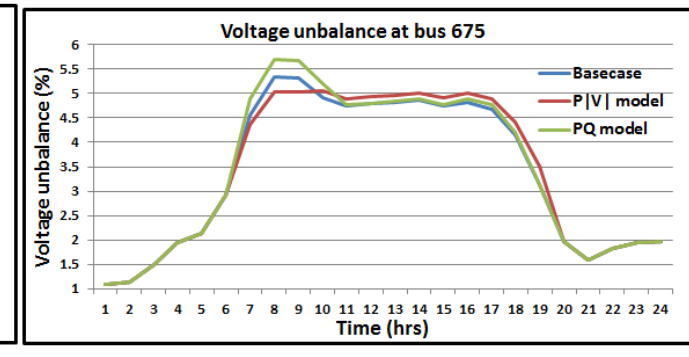

(b) Voltage unbalance at bus 675

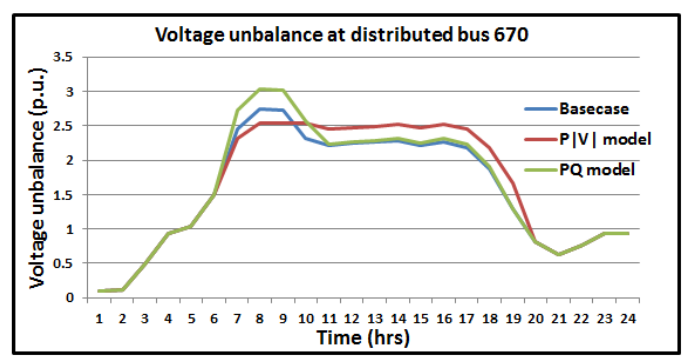

(c) Voltage unbalance at bus 670

Figure 4.10: Voltage unbalance at 634, 675 and 670 buses

ance occurs during the peak demand period and peak sun hours which happens during the mid-day.

\section{Equipment loading and power losses}

The distribution system loadability is also improved with the PV-DG. As a result of the current reduction by (4.21), the equipment loading capacity has been relieved and the loading margin improved with the PV-DG. This is a pivotal value attribute of the PV-DG because a severe voltage drop occurs at load nodes where the peak loading point is reached [143]. However, in this case, the performance of the $\mathrm{P}|\mathrm{V}|$ model is better than that of 
the PQ model. During the peak period from the school's profile, the P|V| model produced a greater loading margin for the entire feeder than the PQ model as shown in Figure 4.11.

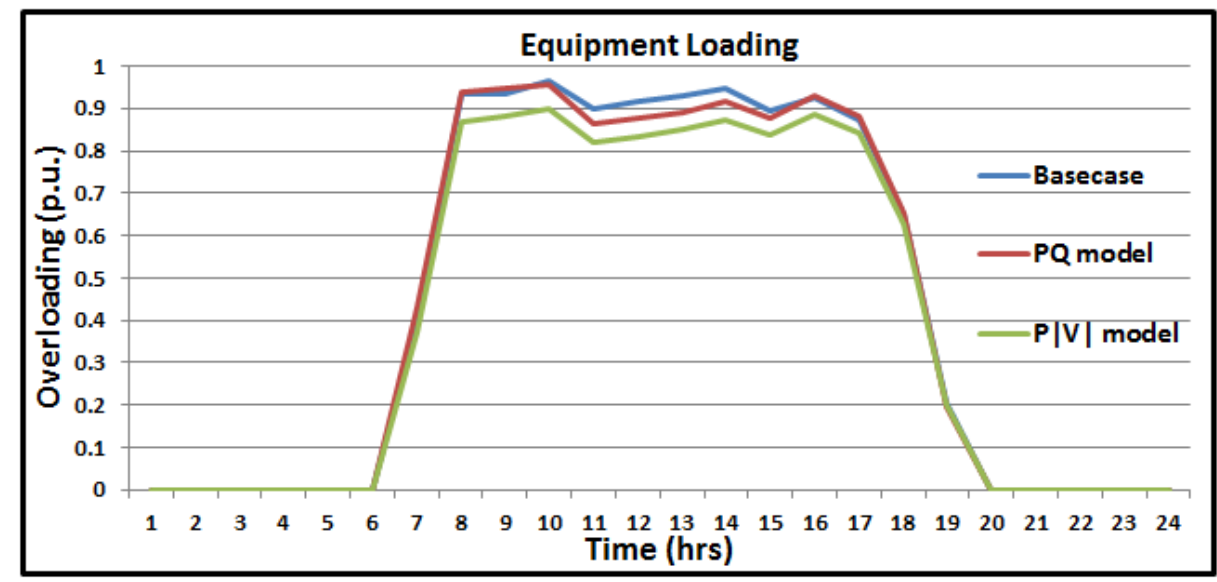

Figure 4.11: PV-DG impact on the equipment overloading

\section{Power losses}

The contribution of distribution system to power losses is about 70\% [144], which makes loss reduction critical in order to improve the utility's energy efficiency. In addition, electrical losses result in high energy demand and increase in load current across system components which can eventually cause a rise in the cost of electricity generation [139]. Moreover, from the load current offset by (4.21), the power losses in the distribution grid due to power flow through the loads, lines and transformers are reduced. The $\mathrm{P}|\mathrm{V}|$ model produced lower active, reactive load and line losses than the PQ model as shown in Figures 4.12.

\section{Computational cost}

The total number of iterations for a converged power flow solution using the $\mathrm{P}|\mathrm{V}|$ generator model was two iterations while the PQ model converged after four iterations. Also, the number of iteration for control operations was one iteration for the $\mathrm{P}|\mathrm{V}|$ model and two iterations for the PQ 


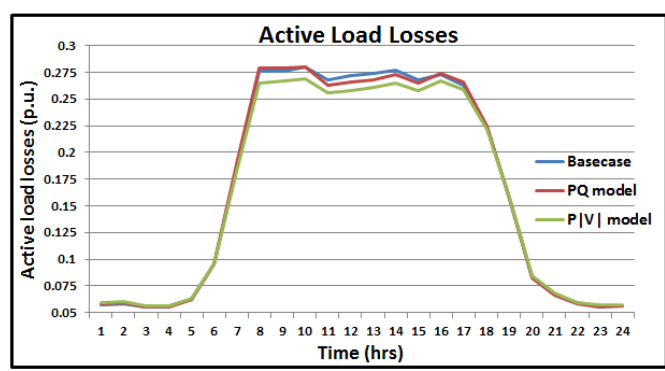

(a) Active load losses

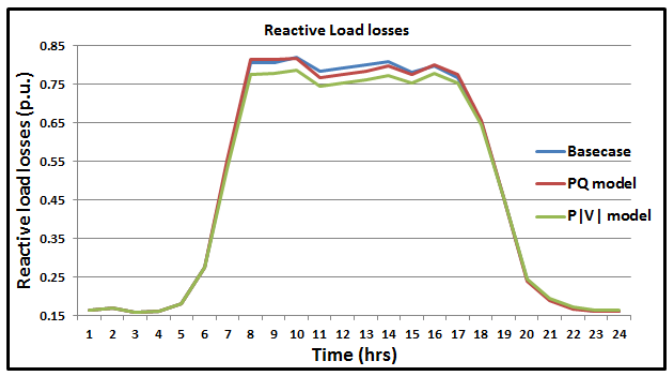

(b) Reactive load losses

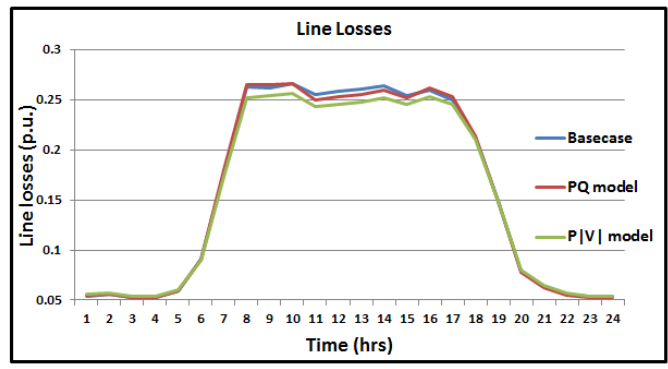

(c) Line losses

Figure 4.12: Feeder power losses 
model. This is because the $\mathrm{P}|\mathrm{V}|$ bus has a regulation scheme for maintaining the specified voltage magnitude within the reactive power limits.

\subsection{Chapter summary}

In this chapter, impacts of single-phase PV-DG models distributed in the unbalanced active IEEE-13 distribution test network are investigated. A five-minute demand profile and solar irradiance data are incorporated in order to show the effects of the time-varying nature of load and solar intermittency.

Results obtained show that the $\mathrm{P}|\mathrm{V}|$ generator model performs better than the traditional PQ model for PV-DG integration with the distribution network in terms of equipment loading, feeder current, voltage profile and power losses offset. Although single-phase PV-DG would normally lead to increase in voltage unbalance, this also is a function of size, location, generator model and load situation. From the results obtained, $\mathrm{P}|\mathrm{V}|$ model performs better during the early morning hours when the school's demand surges and maintains a value above the basecase, while the PQ model increased the unbalance over the time period with a significant spike during early morning hours. Also, the $\mathrm{P}|\mathrm{V}|$ model converges quickly than the traditional PQ model with reduced number of iterations lowering the computational cost of analysing the evolving active distribution network for power system planners.

In addition, it is observed that since the school's demand and solar generation peaks match, the power produced from the solar PV can be used to offset the local and system-wide demand especially during peak hours and therefore, helps to reduce the burden on the power system and enhance its reliability while creating an energy self-sufficient society. 


\subsection{Next chapter}

The capacity value of PV system is a function of the coincidence of its output and load demand. The next chapter presents a proposed method for the computation of the incremental capacity additions and measurement of the period of upgrade deferral provided by PV systems to the distribution system. These analysis are carried out using the IEEE-13 and 34 bus networks representing typical heavily and lightly loaded systems. 
Chapter 4 


\section{Chapter 5}

\section{Grid capacity released analysis and incremental addition computation for planning}

This chapter presents a network released capacity analysis for the power delivery system of the distribution grid, and the computation of the incremental capacity addition provided by an optimally deployed photovoltaic (PV) system in the IEEE 13 and 34 test feeders. Also, in this chapter a method capable of measuring risk and upgrade deferral in the evolving active distribution system planning (ADSP) using two energy quantities, Energy Exceeding Normal (EEN) and Unserved Energy (UE), is presented. The results in this study have been have been peer-reviewed and published in [21], [22]

\subsection{Introduction}

The intrinsic strong correlation that exists between network capacity and reliability gives the inclusion of renewable distribution generation (DG) and the evaluation of their additional load serving capacity a prime place in the emerging ADSP [15], [145]. 
The evolution of conventional planning practices is pivotal in order to accelerate the graceful adoption of this new technology in the electric power system (EPS). This is a very fundamental step with the most strategic impact in distributed generation (DG) uptake. Furthermore, to allow full integration of these intermittent power sources, their inclusion at early stages during the planning process and demand growth forecast is very important [146].

However, with the growth and increasing presence of PV-DG within the area EPS, utilities are still not clear as to how DG are to be treated in the planning process, and the computation of their incremental capacity additions remains a critical issue [15]. Three major factors in distribution system planning include peak load serving capacity, cost and reliability of service [147]. Also, having sufficient capacity to provide peak load relief remains an age-long problem in distribution planning [148]. Although the planning value of renewable DG is a function of time and location, however, the major challenge is the computation of the additional load serving capacity provided by these power sources [16], [145].

The existing literature proposes different metrics for determining the capacity contributions of variable power sources such as the equivalent conventional power (ECP), peak load carrying capability (PLCC), effective load carrying capability (ELCC), equivalent firm power (EFP), load duration capacity (LDC) and demand time matching (DTIM) [149], [150], [151], [152], [153]. Garver [154] estimated the ELCC of generating units by introducing a new parameter which is used to characterize the loss-ofload probability as a function of reserve megawatts. Once the parameter is determined, the ELCC of the new generating system can be related to its rating and forced outage rate. Abudullah et al. [147] presented the capacity deferral credit computation of renewable DG systems using a modified load adjustment method to determine their ELCC. Also, in another study, Abudullah et al. [149] proposed a noniterative analytical method to determine the PLCC and ELCC of conventional and renewable power 
sources. The result showed that the higher the correlation between load demand and renewable generation units, the higher the ELCC, and vice versa. Dragoon et al. [155] presented a simplified analytical probabilistic method which maintains the network resource adequacy and evaluates the peak load carry capability of the incremental generating systems.

D'Annunzio et al. [156] proposed a non-iterative approach used to evaluate the ELCC of a wind farm and Hasche et al. [157] presented a methodology to identify the minimum amount of data needed to compute the capacity value of wind power. Madaeni et al. [152], [158], [159] presented a comparison of methods such as the computationally intensive statistical and approximation approaches used to compute the capacity value of solar PV systems. Analysis presented showed that some approximation methods produced similar ELCC when compared with the reliability-based methods.

Other related studies in [17], [160], [161] have also computed the capacity value of renewable DG such as wind and solar PV using the ELCC metric which demands substantial system reliability modelling and estimation of detailed reliability-based indices.

Further, the extant literature presents capacity value computation in terms of ELCC without considering the impact of integrating renewable DG systems on the loading (ratings) of the power delivery system. Violating capacity constraints of the power deliver elements (PDE) such as lines, loads, transformers and switches can degrade network reliability. This is due to the strong correlation that exists between capacity and network reliability, especially if the PDE loading is close to system constraints [15]. Also, utility distribution planners may not be too comfortable with absolute probabilistic planning or approximation approaches but would rather prefer to subscribe to concrete limits [162].

This study proposes a method capable of computing the incremental capacity additions, measuring risks and upgrade deferral provided by PV systems deployments. This method includes surrogate metrics, energy 
exceeding normal rating and unserved energy, for evaluating system reliability and capacity usage. The contributions of this chapter is as follows:

- Sensitivity analysis for optimal deployment of a PV-DG plant on IEEE 13 and 34 test feeders.

- Risk and upgrade deferral measurements for both networks using energy quantities - EEN and UE.

- Evaluation of the additional load serving capacity provided by the PV-DG for network planning.

\subsection{Energy quantities for incremental capacity mea- surement}

The two fundamental energy metrics used in measuring risk and the impact on system capacity are [15], [148], [16], [145], [162], [163]:

- Energy Exceeding Normal (EEN): This is the energy served above the "Normal" limits or ratings of the PDE over a certain period of time as shown in Figure 5.1. As an index value used to trigger plan-

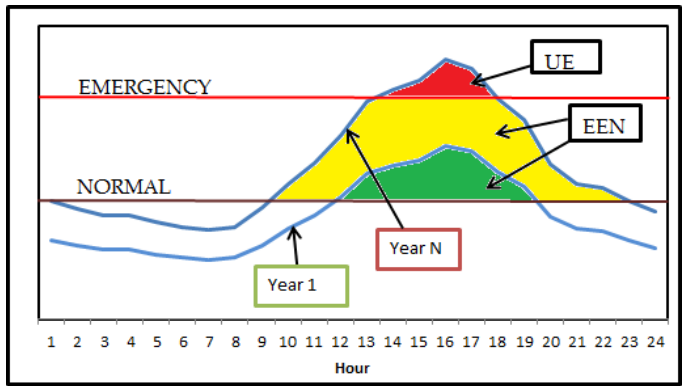

Figure 5.1: Energy Exceeding Normal (EEN) and Unserved Energy (UE) concepts

ning studies, it is a key metric for assessing the potential value of 
a renewable DG integrated with the area EPS. Also, it is referred to as the energy at the risk of being unserved when the "Normal" firm limit is violated. In addition, EEN plots show how much of the system capacity is being utilized. Furthermore, it is important to note that the EEN index does not provide a direct assessment of system reliability, however, it is referred to as a surrogate for evaluating reliability of the design utilized by distribution planners. The "Normal" limit is used in comprehensive planning studies to evaluate the risk of reliability issues with the PDE.

- Unserved Energy (UE): This is the energy estimated to exceed the "Emergency" or "Maximum" rating or limits of the power delivery system as shown in Figure 5.1. The maximum rating, with little engineering tolerance, once violated leads to load shedding and UE, which has to be curtailed immediately to reduce power to a convenient level. Moreover, from the EEN and UE plots, distribution planners can visualize the nature and degree of the capacity problem being faced.

The energy meter connected at the feeder head sweeps along its zone and queries the PDEs for power values above the normal and maximum limits. Further, the EEN and UE are calculated by computing the amount of power exceeding the PDE ratings in comparison with the normal and emergency ratings. The power obtained is then integrated to get energy values [15], [162].

\subsection{System released capacity and loss analysis}

Power delivery system overload and losses depend largely on the RMS value of the current flow through the PDE. An overload condition is more likely to occur with the peak values of the current while all values of current contribute to the total system losses [33]. However, a good PV-DG 
application within the traditional electricity grid can result in system capacity relief (or release) in terms of overload and power loss reduction through current offset. This has been shown in the previous chapter from equation (4.17) to (4.19).

Also, the current reduction ensures that the maximum power flow after PV-DG integration is less than the basecase and therefore, avoiding the violation of the overload limits of the PDE. This makes PDE capacity release and loss reduction possible with an optimal PV-DG application through current injections at different penetration levels. Furthermore, for a branch $j$ on a radial feeder with an active power flow only, the total real power flow before PV-DG integration is [33]:

$$
P_{j}(t)=P_{j}^{d e m}(t)-P_{j}^{g e n}(t)
$$

In order to prevent an overload of the power delivery system after PV-DG connection the following criterion must be satisfied:

$$
P_{j}^{\text {pvmax }}(t)<P_{j}^{\text {dem }}(t)
$$

This implies that the maximum active power flow with PV-DG present should be less than the basecase demand to prevent an overload of the power delivery element.

where $P_{j}(t)$ is the total real power flow through branch $j, P_{j}^{\text {dem }}(t)$ represents the total downstream real power demand at branch $j, P_{j}^{g e n}(t)$ is the total downstream real power produced at branch $j$ and $P_{j}^{\text {pvmax }}(t)$ indicates the PV total downstream real power injection at branch $j$.

In addition, considering a scenario of both real and reactive power flow for a section of the distribution network, the permissible (apparent) power without PV-DG is given as:

$$
S_{k, W O P V}=P_{k}+j Q_{k}=\sqrt{P_{k}^{2}+Q_{k}^{2}}
$$

With PV-DG present, the apparent power becomes: 


$$
S_{k, W P V}=\sqrt{\left(P_{k}-P_{P V}\right)^{2}+\left(Q_{k}-Q_{P V}\right)^{2}}
$$

The risk of power delivery system overload is reduced with the penetration of PV-DG as long as the following expression is satisfied:

$$
S_{k, W P V}<S_{k, W O P V}
$$

Also, equation (5.4) shows that the condition in (5.5) can be easily satisfied if power injections are optimized, i.e., values of $\left(P_{k}-P_{P V, k}\right)$ and $\left(Q_{k}-\right.$ $\left.Q_{P V, k}\right)$ are minimum.

\subsection{Optimal sizing and placement of PV-DG}

\subsubsection{Optimal sizing}

In order to obtain the optimal size of the PV-DG which ensures that power losses are minimized at any bus $k$ on the distribution network, the partial derivative of the apparent power loss with respect to the active power injections from the PV-DG at the specified bus is calculated as follows [138], [164]:

$$
P_{\text {loss }}=\sum_{k=1}^{N} \sum_{m=1}^{N}\left\{\alpha_{k m}\left[P_{k} P_{m}+Q_{k} Q_{m}\right]+\beta_{k m}\left[Q_{k} P_{m}-P_{k} Q_{m}\right]\right\}
$$

Equation (5.6) is commonly referred to as the "exact loss" formula. The reactive power injections from the PV-DG can be expressed in terms of the active power injections as given in (5.7)

$$
Q_{P V, k}=\mu P_{P V, k}
$$

where:

$\mu= \pm \tan \left[\cos ^{-1}\left(P F_{p v, k}\right)\right]$, positive $\mu$ implies the PV-DG is injecting reactive power and negative for absorbing reactive power, and $P F_{p v, k}$ is the PV-DG 
operating power factor at bus $k$.

The real $\left(P_{k}\right)$ and reactive power $\left(Q_{k}\right)$ injections at bus $k$ are given as follows:

$$
\begin{gathered}
P_{k}=P_{P V, k}-P_{l o a d, k} \\
Q_{k}=Q_{P V, k}-Q_{l o a d, k}=\mu P_{P V, k}-Q_{l o a d, k}
\end{gathered}
$$

where $P_{\text {load,k }}$ and $Q_{\text {load,k }}$ are the load active and reactive power at bus $k$ respectively. Substituting Eqs. (5.8) and (5.9) in (5.6), it becomes:

$$
\begin{array}{r}
P_{\text {loss }}=\sum_{k=1}^{N} \sum_{m=1}^{N}\left\{\alpha_{k m}^{p}\left[\left(P_{P V, k}-P_{\text {load }, k}\right) P_{m}+\left(\mu P_{P V, k}-Q_{\text {load }, k}\right) Q_{m}\right]\right. \\
\left.+\beta_{k m}^{p}\left[\left(\mu P_{P V, k}-Q_{\text {load }, k}\right) P_{m}-\left(P_{P V, k}-P_{\text {load }, k}\right) Q_{m}\right]\right\}
\end{array}
$$

The injection of power from the PV-DG is expected to reduce power flow and system losses. However, if the power supply from the PV unit is greater than the optimal size, a reverse power flow condition occurs which could affect the operation of the network protecting and voltage regulating devices [30].

PV-DG size is varied in MATLAB and OpenDSS environment to determine its optimal size subject to voltage and basecase loss constraints. At each bus location, the variation of the DG size produces a power loss variation pattern. As depicted in Figure 5.2, remote buses produced greater loss reduction than buses close to the feeder source as DG sizes are varied. This shows that PV-DG siting on the feeder determines system loss minimization. Optimal DG sizes at various buses are obtained at the point of minimal losses in the curves.

Also, as depicted in the plot, the optimal DG sizes varied with each bus and thus, showing that DG deployments have locational impact on the variation of power loss. 


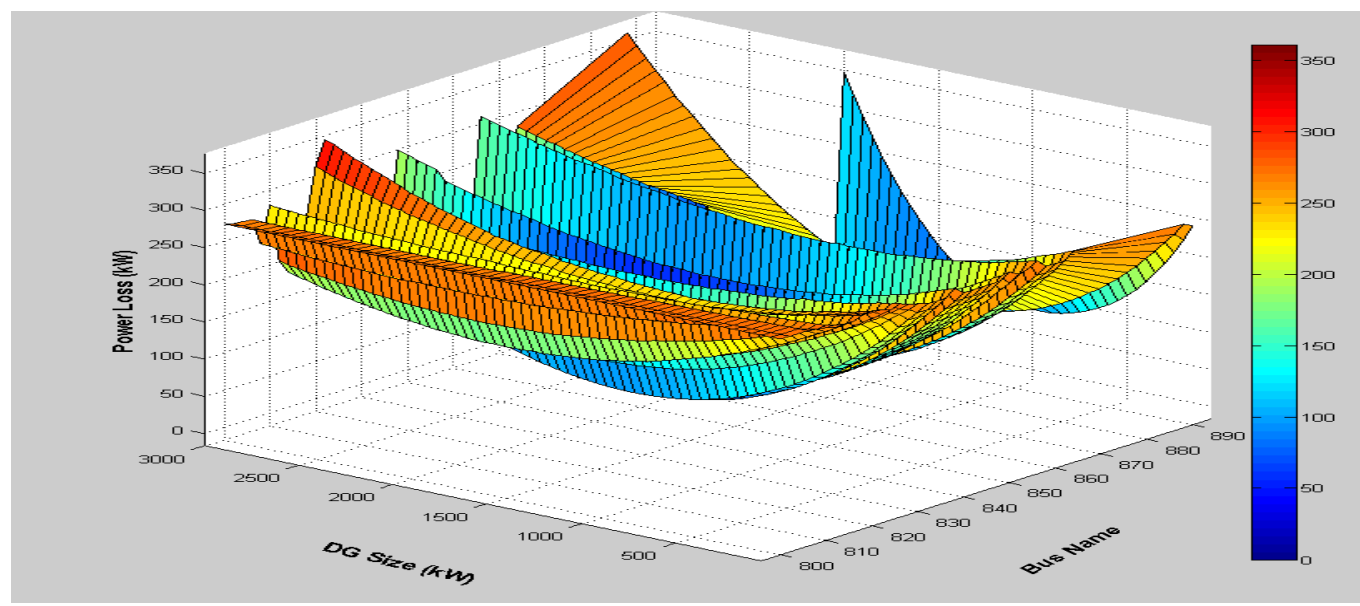

Figure 5.2: Power loss variation curves for all buses on the test feeder

\subsubsection{Optimal placement}

The problem of determining the most suitable site for DG placement can be formulated as a minimization of power losses [165], [166]. This is defined as:

$$
\operatorname{minimize} \Sigma\left(P_{L}+j Q_{L}\right)
$$

Subject to the following constraints:

$$
\begin{aligned}
P_{G k}-P_{L k}=P_{k} & \text { active power balance at bus } \mathrm{k} \\
Q_{G k}-Q_{L k}=Q_{k} & \text { reactive power balance at bus } \mathrm{k} \\
V_{k, \text { min }} \leq V_{k} \leq V_{k, \max } & \text { voltage level at bus } \mathrm{k} \\
\sum_{k m} P_{\text {loss }, G} \leq \sum_{k m} P_{\text {loss }} & \text { real loss limit in the line from bus } \mathrm{k} \text { to } \mathrm{m} \\
\sum_{k m} Q_{\text {loss }, G} \leq \sum_{k m} Q_{\text {loss }} & \text { reactive loss limit in the line from bus } \mathrm{k} \text { to } \mathrm{m} \\
i_{k m} \leq i_{k m, \max } & \text { line current limits from bus } \mathrm{k} \text { to } \mathrm{m} \\
S_{k m} \leq S_{k m, \max } & \text { branch power flow limits from bus } \mathrm{k} \text { to } \mathrm{m}
\end{aligned}
$$

where $P_{G k}$ and $Q_{G k}$ represent active and reactive power generation respectively at bus $k$. $P_{L k}$ and $P_{L k}$ are active and reactive loads respectively at 
bus $k . P_{l o s s, G}$ and $Q_{l o s s, G}$ are active and reactive power losses respectively with DG. $P_{\text {loss }}$ and $Q_{\text {loss }}$ are active and reactive power losses respectively without DG.

Further, two sensitivity indices have been used to optimally site the PV-DG on the distribution test systems. They are the voltage sensitivity index (VSI) and loss sensitivity index (LSI). The sensitivity data, used for network conceptualization of the impact of system modifications, can be obtained from the Newton-Raphson load-flow and perturb-and-observe algorithms [167], [168], [169]. The former is an iterative load-flow technique which provides sensitivity data from the inverse of the standard Jacobian matrix used to estimate the network bus voltages, given as:

$$
\left[\begin{array}{c}
\Delta \delta \\
\Delta V
\end{array}\right]=\left[\begin{array}{ll}
\frac{\partial P}{\partial \delta} & \frac{\delta P}{\partial V} \\
\frac{\partial Q}{\partial \delta} & \frac{\delta Q}{\partial V}
\end{array}\right]^{-1}\left[\begin{array}{l}
\Delta P \\
\Delta Q
\end{array}\right]
$$

where $\Delta \delta$ and $\Delta V$ are the absolute changes in phase and voltage respectively. $\Delta P$ and $\Delta Q$ are the active and reactive power injections.

However, the classical Newton-Raphson approach is computationally complex [169], does not allow the calculation of sensitivities against positions of transformer tap-changers [168] and with sensitivity data seldom accessible in modern power system planning software [167]. On the other hand, although the perturb-and-observe algorithm takes time due to the need to re-compute the system state for every modification at each bus, it uses existing models and simulators while allowing more applicationspecific and time-saving simulation approaches to be chosen [167]. Therefore, in this study, the perturb-and-observe network algorithm, as documented in Algorithm 1 [167], is used to generate the voltage $\left(\partial|V| / \partial P_{k}\right.$ and $\left.\partial|V| / \partial Q_{k}\right)$ and loss $\left(\partial P_{L} / \partial P_{k}\right.$ and $\left.\partial Q_{L} / \partial Q_{k}\right)$ sensitivities. 


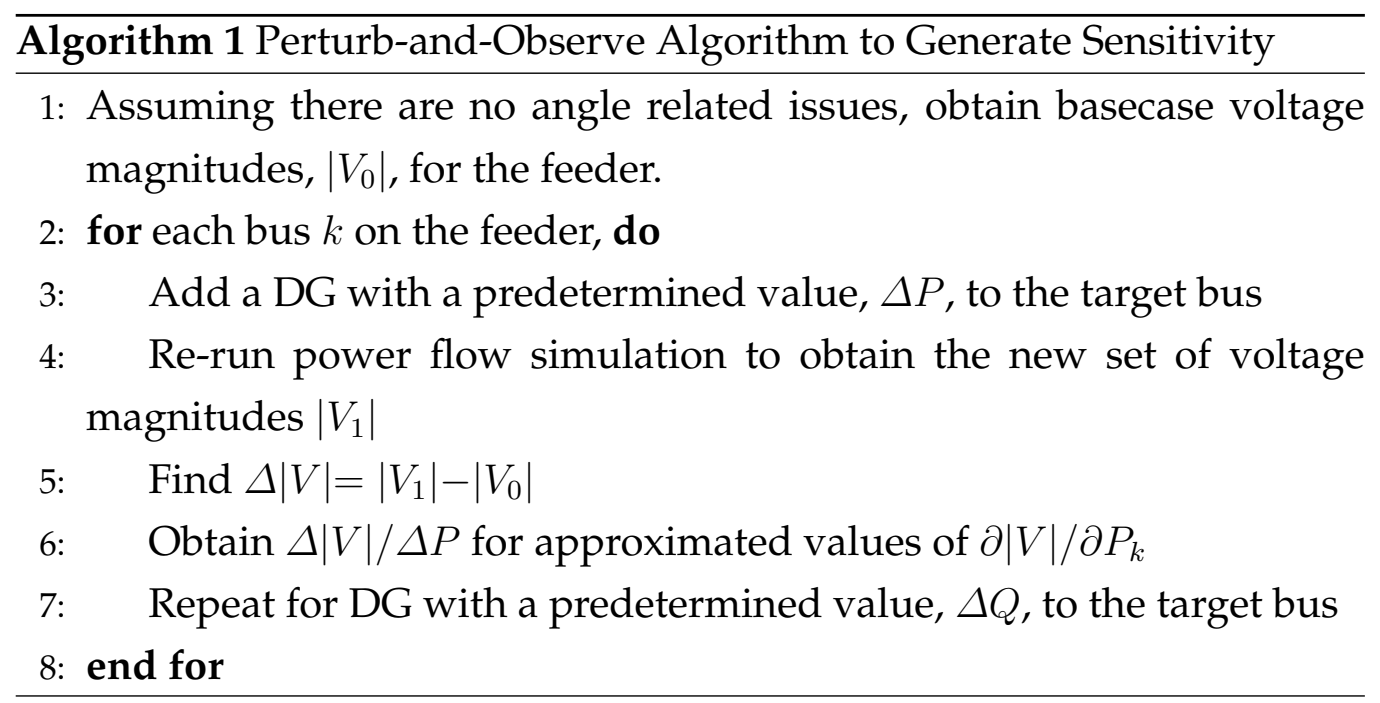

\subsection{Annual load and irradiance characteristics}

\subsubsection{Yearly load characteristics}

Annual simulations are carried out using a commercial demand profile (obtained from the Electric Power Research Institute (EPRI) [162]) as shown in Figure 5.3, representing daily energy usage in p.u. for each month of the year. Also, there are significant peaks in the load curve at the beginning and middle of the year. These occur mostly during the middle of the day, signifying that the entire system would benefit from a DG (such as the solar PV-DG) capable of offsetting peak demand during the middle of the day.

\subsubsection{Solar generation characteristic}

Figure 5.4 shows an annual irradiance characteristic (obtained from [170]) with consistently significant peaks during the middle of the day. The load and irradiance profiles coincides approximately well, signifying that the PV-DG output can provide significant capacity relief for power delivery systems in the distribution network. 


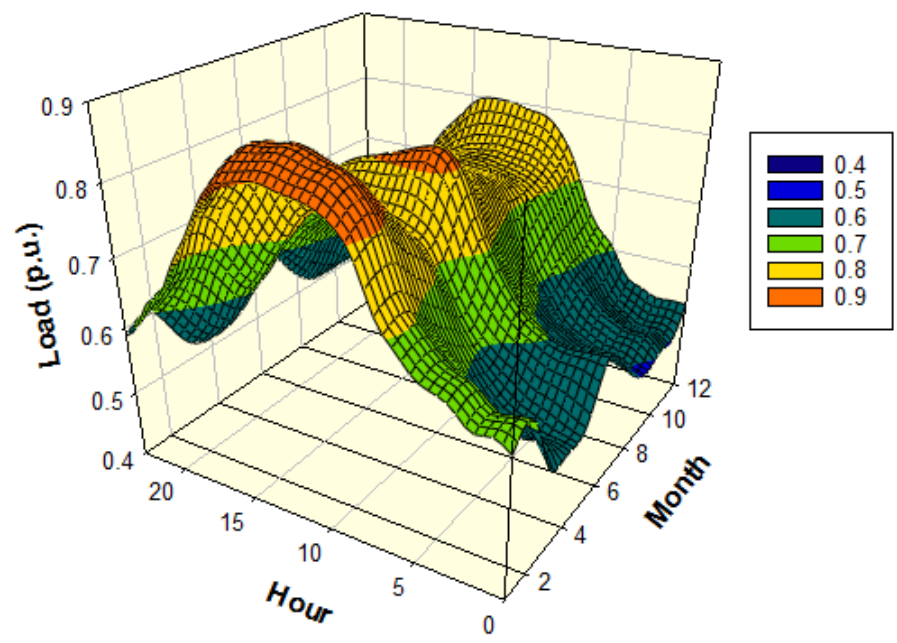

Figure 5.3: Annual energy demand profile

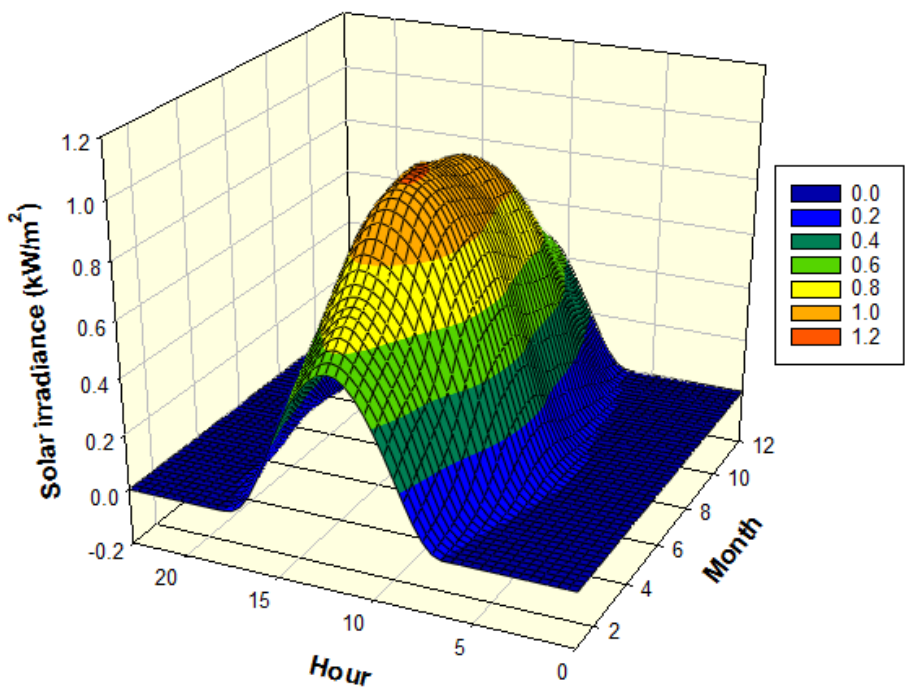

Figure 5.4: Annual irradiance profile 


\subsection{Case study and simulation results}

\subsubsection{IEEE 13-bus test feeder}

The buses in the test network (depicted in Figure 5.5) are ranked using the loss and voltage sensitivity indices in order to optimally site the PV-DG system on the network as shown in Figure 5.6.

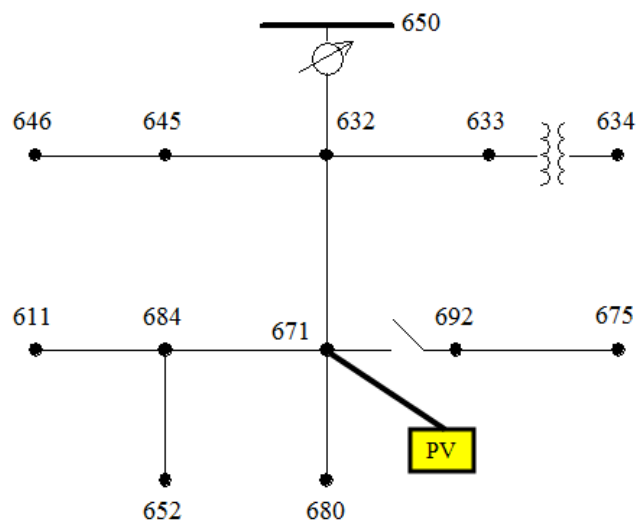

Figure 5.5: Modified IEEE 13-bus test feeder. The modification refers to the presence of PV-DG

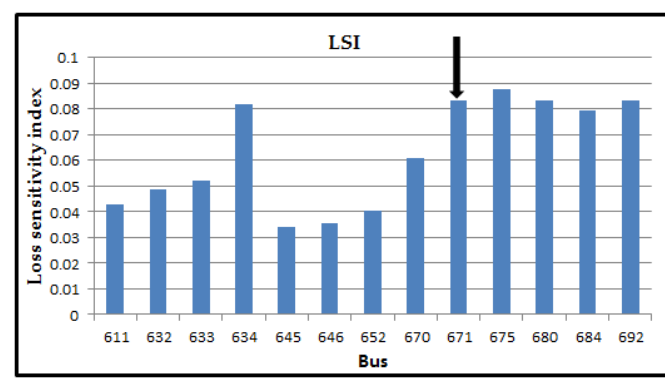

(a) Loss sensitivity

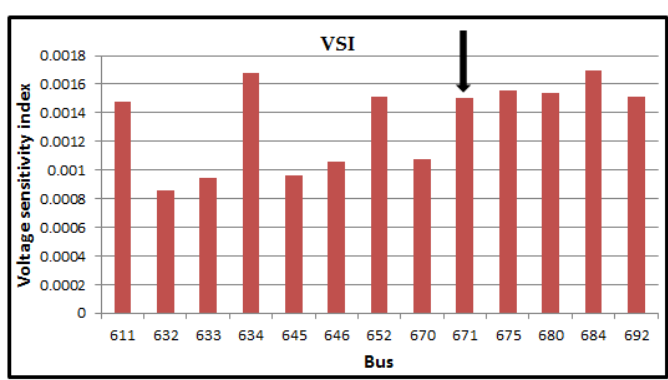

(b) Voltage sensitivity

Figure 5.6: Loss and voltage sensitivities

Bus 671 is selected, although not the highest ranked node, because it is a 3-phase delta connected spot load on the network, and a three-phase 
centralized PV unit is installed at this bus.

\subsubsection{IEEE 34-bus test feeder}

Also, simulations are carried out on the IEEE-34 distribution test feeder as shown in Figure 5.7 with a total load of $1769 \mathrm{~kW}$ and $1044 \mathrm{kVAr}$ [171] and following the load profile as shown in Figure 5.3. This test feeder, with spot and distributed loads, is a long lightly loaded system located in Arizona. In addition, it has two different operating voltages of 24.9 $\mathrm{kV}$ and $4.16 \mathrm{kV}$ [171]. With loss and voltage sensitivity indices, bus 890

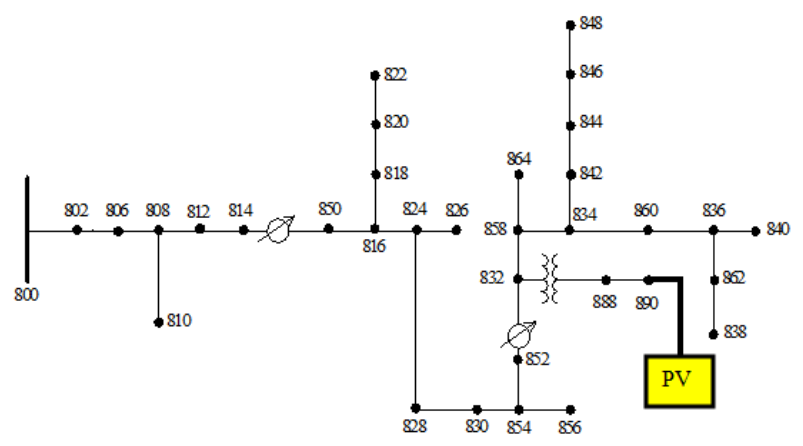

Figure 5.7: Modified IEEE 34-bus test feeder. The modification refers to the presence of PV-DG

located towards the end of the feeder emerged as the highest ranked node and would be referred to as the "sweet or helpful spot" on the test system as depicted in Figure 5.8.

\subsection{Simulation results and discussion}

In order to evaluate capacity contributions of the PV-DG to the distribution network at different penetration levels, a multiyear simulation study considering a yearly load growth is carried out on the IEEE 13 and 34 test 


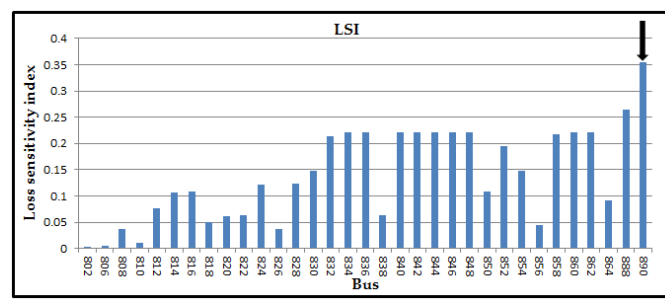

(a) Loss sensitivity

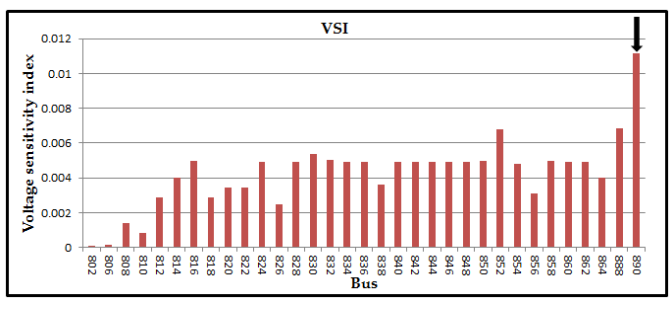

(b) Voltage sensitivity

Figure 5.8: Loss and voltage sensitivities

systems. For a typical year, an annual 8760-hour simulation over the complete load curve is performed using the open-source distribution system simulator (OpenDSS).

\subsubsection{Basecase EEN}

The basecase EEN 3-D plots as shown in Figure 5.9 capture all the peak load demand hours over several months and seasons of the year. Also, they indicate periods when the systems are most constrained, which are during the mid day and occurring at the beginning-, mid- and slightly at the end of year.

These plots indicate how much capacity of the power delivery system in the distribution network is being utilized. For IEEE 13-bus as a heavily loaded feeder, Figure 5.9 shows that a greater portion of the system capacity is being used when compared with the IEEE 34 having the same load profile. This makes capacity issues critical for distribution planners in a more heavily loaded distributed system.

The sharpness of these peaks which occurs mostly during the mid-day period suggests that a good PV-DG application has the capacity to offset the demand and provide peak load relief with incremental capacity addition to the network. However, the dynamic nature of the distribution systems with an almost inevitable load growth each year makes a singleyear result insufficient to characterize the network. Figures 5.10 and 5.11 


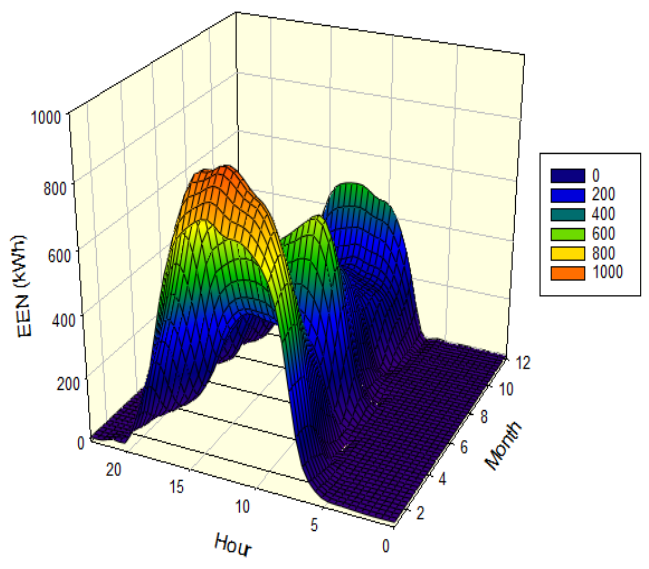

(a) IEEE 13-bus EEN at peak load

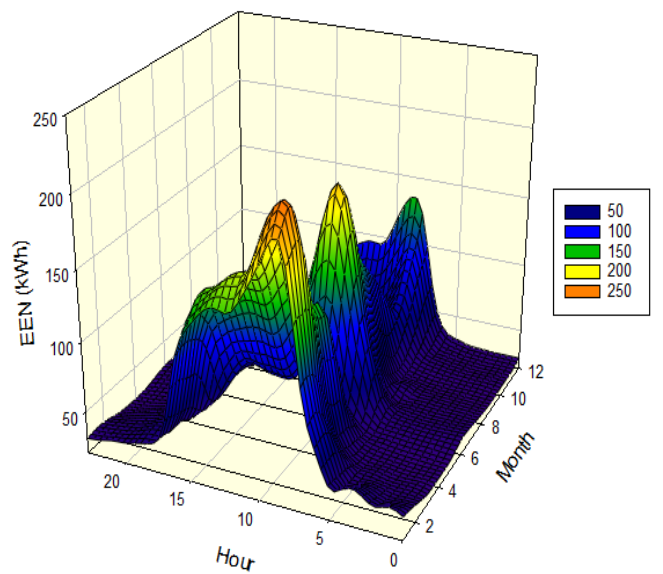

(b) IEEE 34-bus EEN at peak load

Figure 5.9: Basecase EEN at peak load for both systems

depict the outcome of a multiyear planning study of the expected increase of the EEN with a load growth over a 5-year period. Moreover, if distribution planners refuse to take any precautionary step with respect to capacity, the possibility of the EEN being off the scale increases with load growth. These plots are meant to be to be used as visualization tool to help planners for possible system upgrade. In the conventional practice, engineers would plan for infrastructure upgrade to serve the increase in load over the entire period. However, the proliferation of DG such as PV could be deployed to offset demand increase at the levels and periods indicated in these plots. These basecase plots will be used to compare and measure capacity, and system relief benefits of the proposed PV-DG at various penetration levels.

\subsubsection{EEN offset (capacity release) by PV-DG penetration}

This section investigates the impact of the PV-DG integration on the EEN values at various penetration levels for both test systems. 


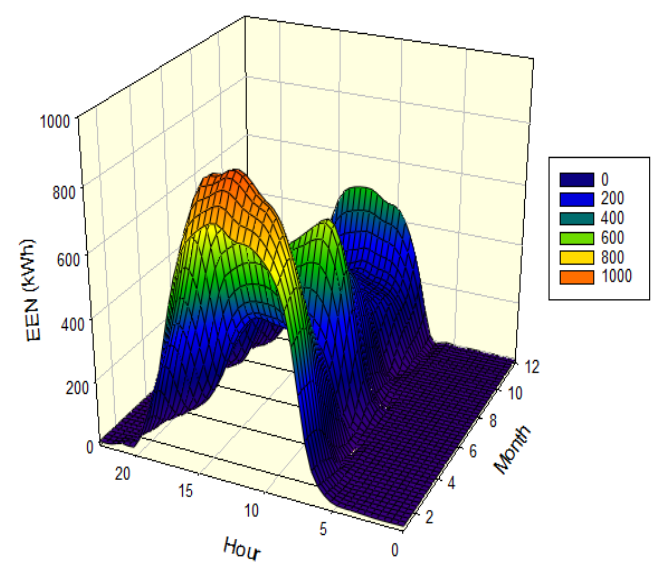

(a) EEN at peak load

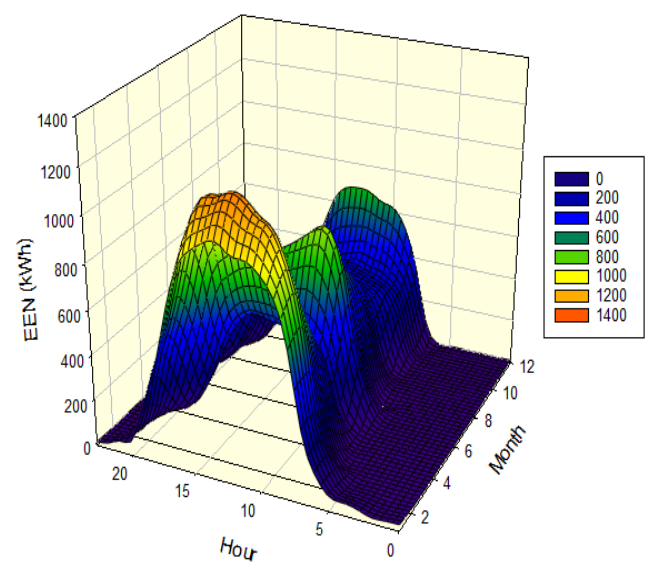

(c) EEN at 1.05 load growth

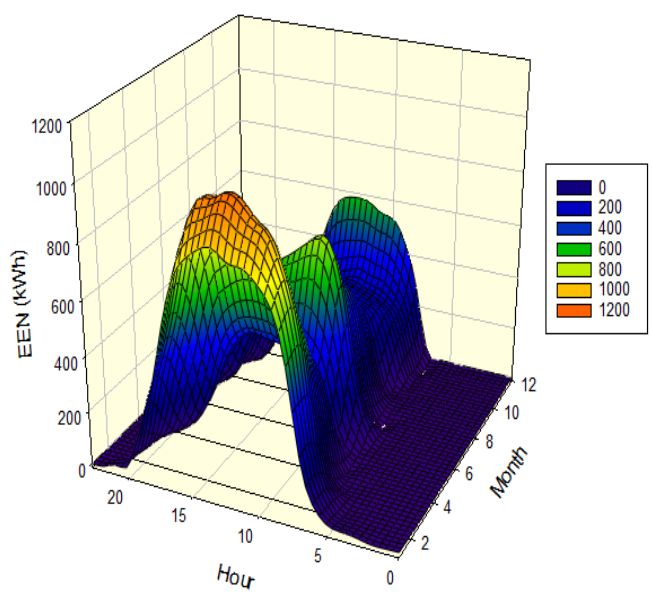

(b) EEN at 1.025 load growth

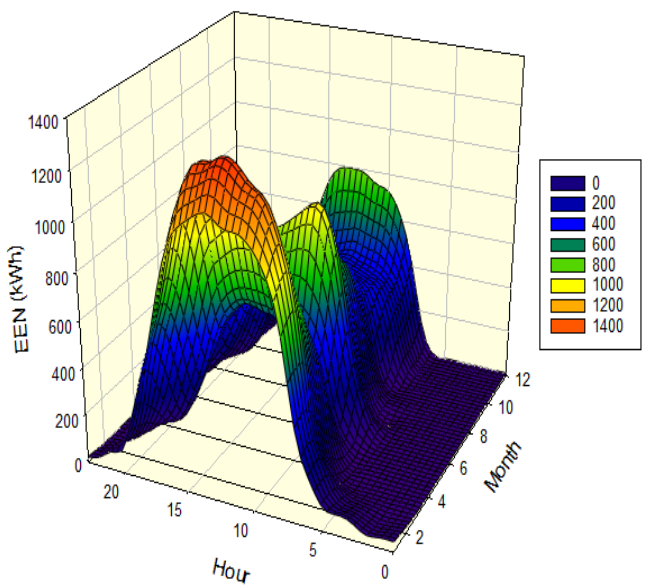

(d) EEN at 1.075 load growth

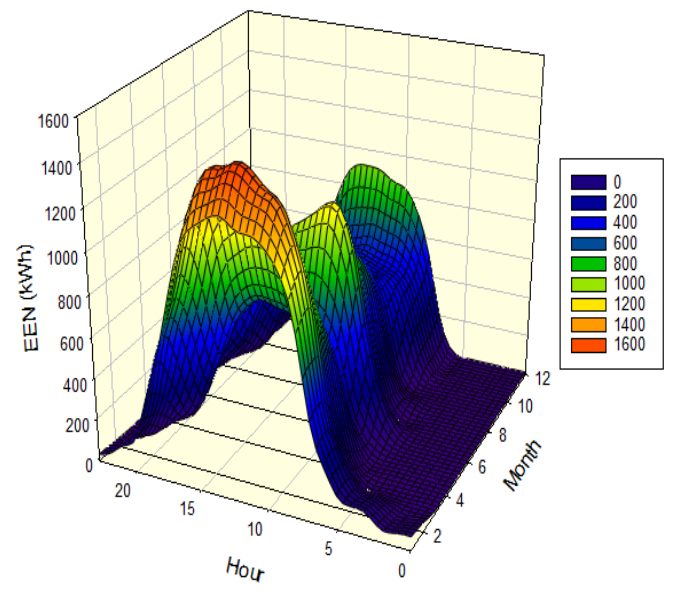

(e) EEN at 1.1 load growth

Figure 5.10: A 5-year period growth of EEN for IEEE 13-bus 


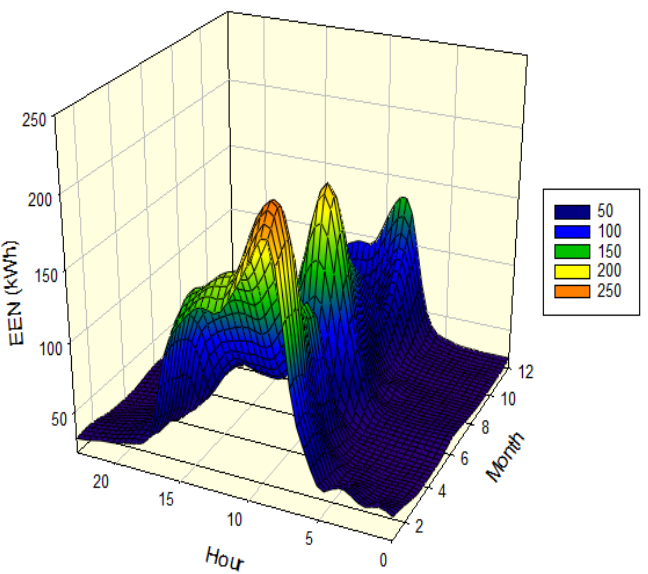

(a) EEN at peak load

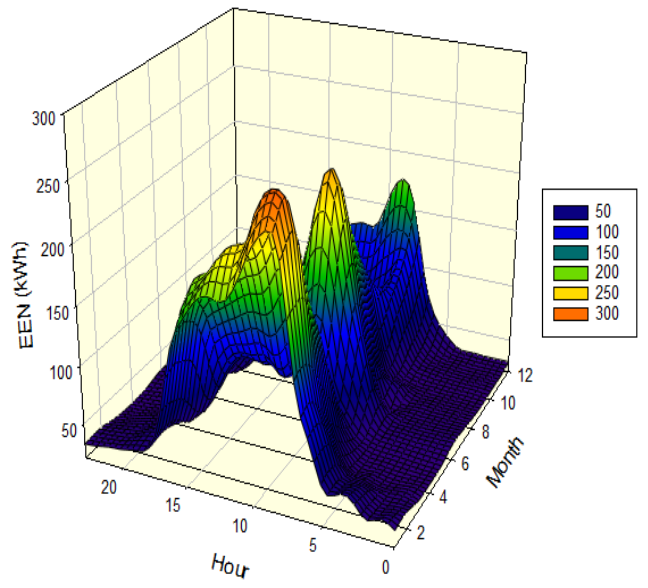

(c) EEN at 1.05 load growth

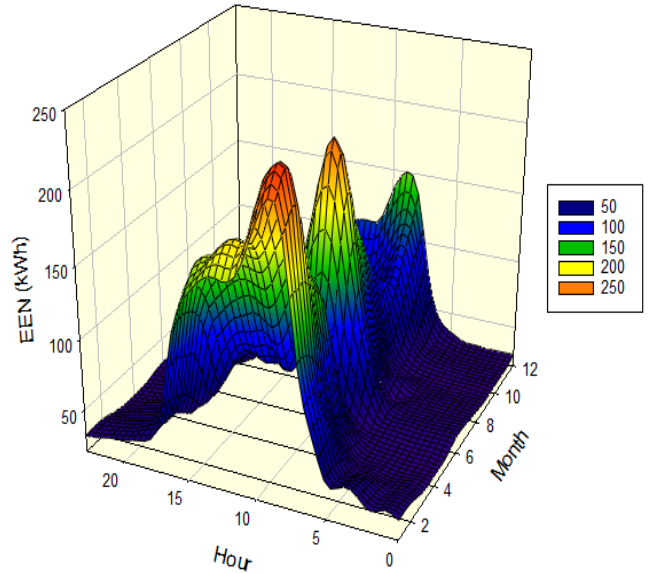

(b) EEN at 1.025 load growth

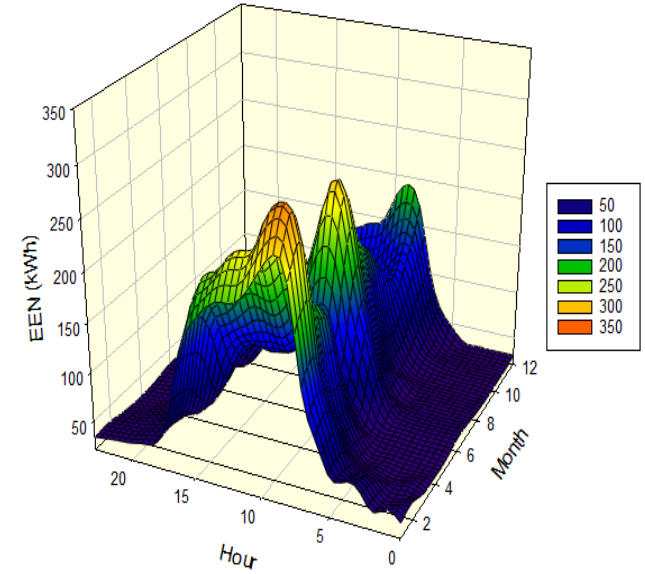

(d) EEN at 1.075 load growth

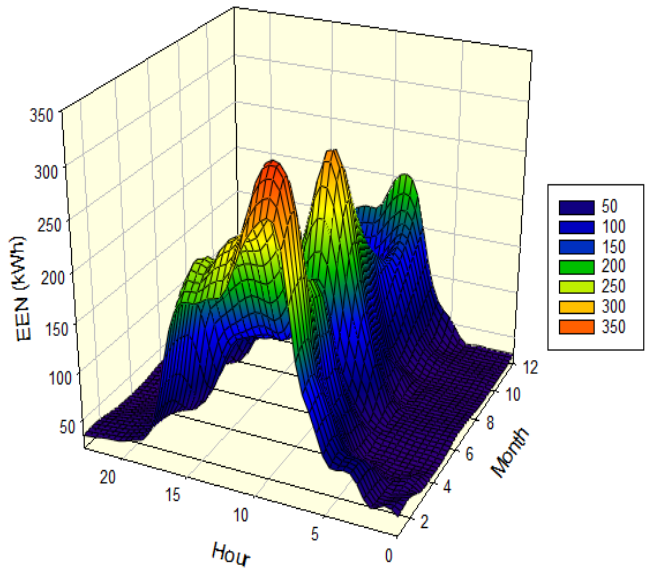

(e) EEN at 1.1 load growth

Figure 5.11: A 5-year period growth of EEN for IEEE 34-bus 


\section{5\% PV-DG penetration}

For both systems, the EEN characteristics reduced significantly as shown in Figures 5.12 and 5.13 at 15\% penetration level. These figures show to what extent this penetration releases capacity (i.e., decreases EEN) in the power deliver system. In comparison with the basecase for the IEEE 13bus, the EEN reduction ranges from $47.3 \%$ to $29.8 \%$ at peak load and 1.1 load growth respectively. Also, for the IEEE 34-bus, the EEN value reduc-

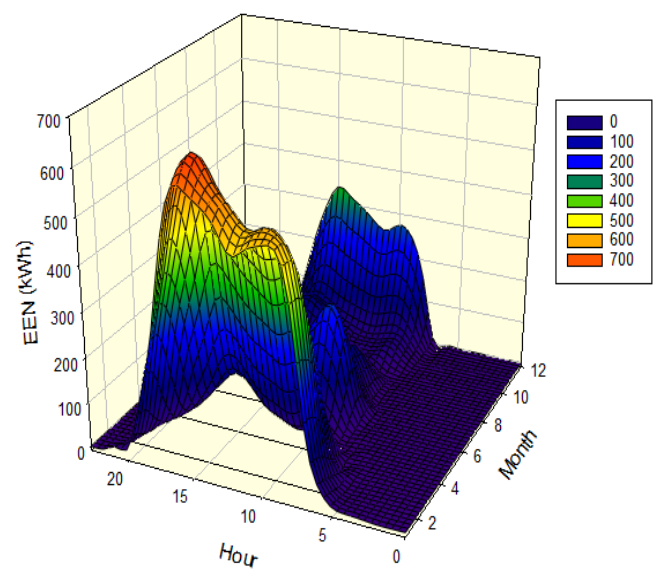

(a) $15 \%$ PV-DG at peak load

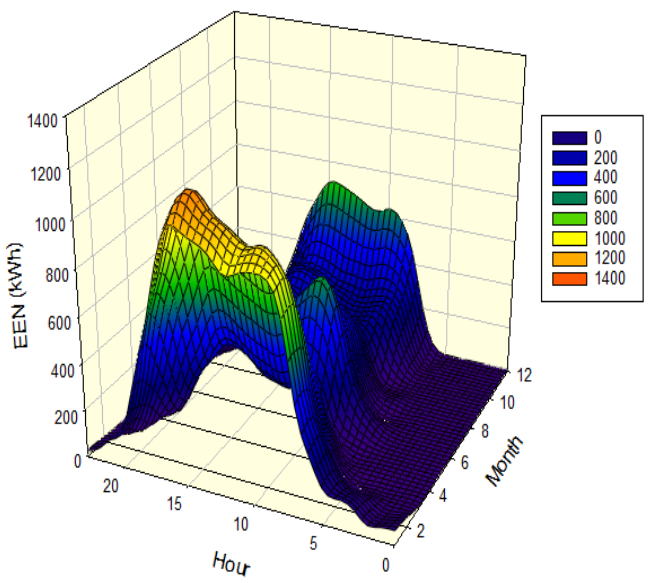

(b) 15\% PV-DG at 1.1 load growth

Figure 5.12: IEEE 13-bus capacity release with 15\% PV-DG

tion ranges from $32.8 \%$ to $42.2 \%$ at peak load and 1.1 load growth respectively.

\section{Optimal size PV-DG}

There is a strong correlation between power losses and system capacity. This implies that deploying PV-DG with optimal size and location to minimize power losses is often near-optimal for system capacity relief issues as well [16]. For both test systems, the EEN values almost flattened out (as depicted in Figures 5.14 and 5.15) during the peak sun hours which occurs mostly between April and September as shown in the irradiance profile in 


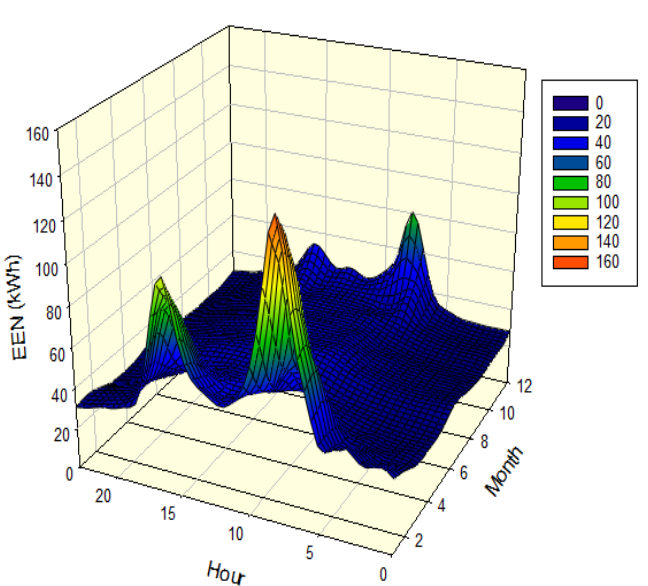

(a) $15 \%$ PV-DG at peak load

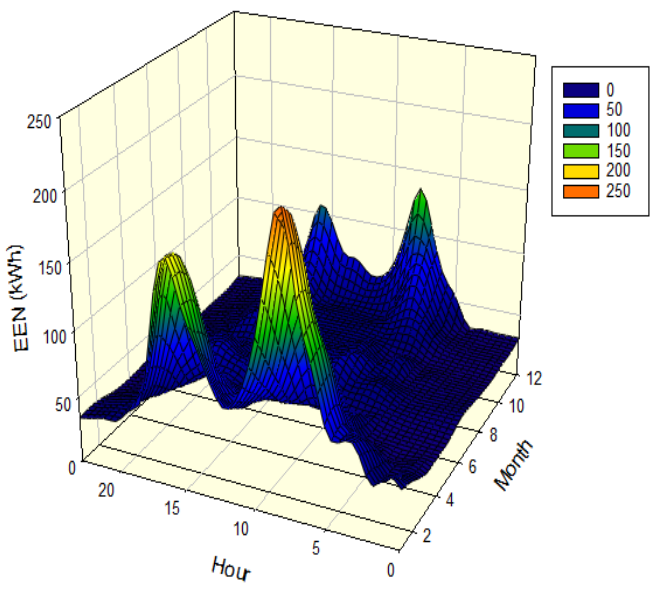

(b) $15 \%$ PV-DG at 1.1 load growth

Figure 5.13: IEEE 34-bus capacity release with 15\% PV-DG

Figure 5.4. The EEN reduction ranges from $90 \%$ to $81.1 \%$ at peak load and 1.1 load growth respectively for IEEE 13-bus system. Also, for IEEE 34-bus

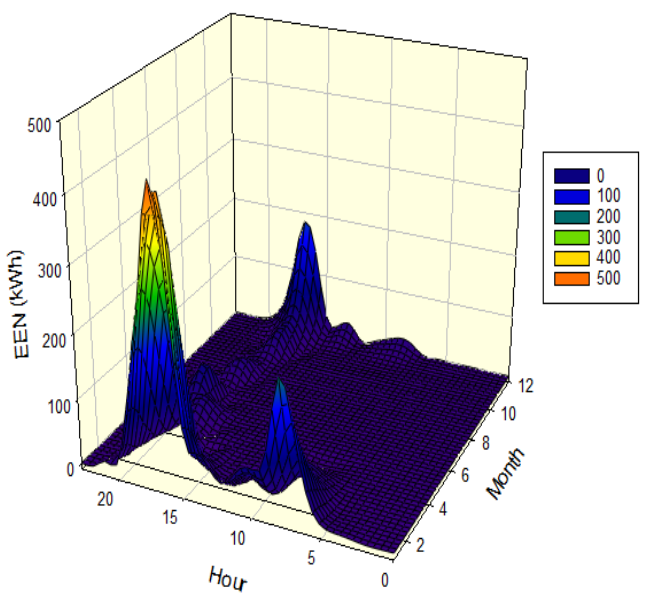

(a) Capacity release at peak load

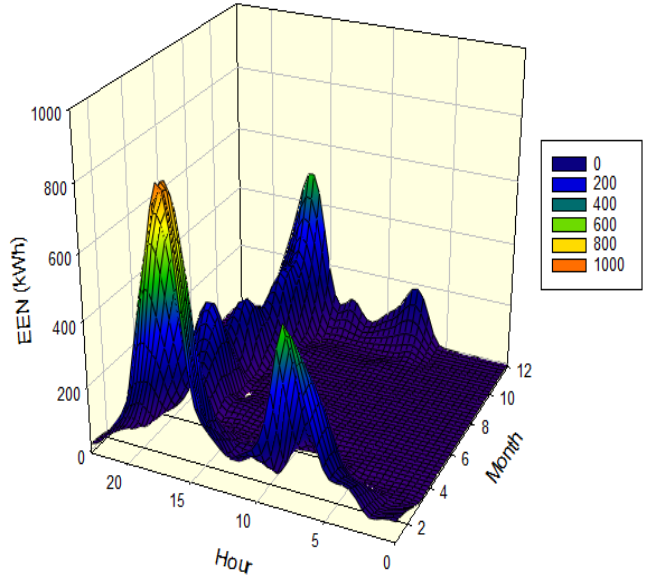

(b) Capacity release at 1.1 load growth

Figure 5.14: IEEE 13-bus capacity release with optimal size PV-DG

system, the EEN value reduction ranges from $34.9 \%$ to $47.1 \%$ at peak load and 1.1 load growth respectively. 


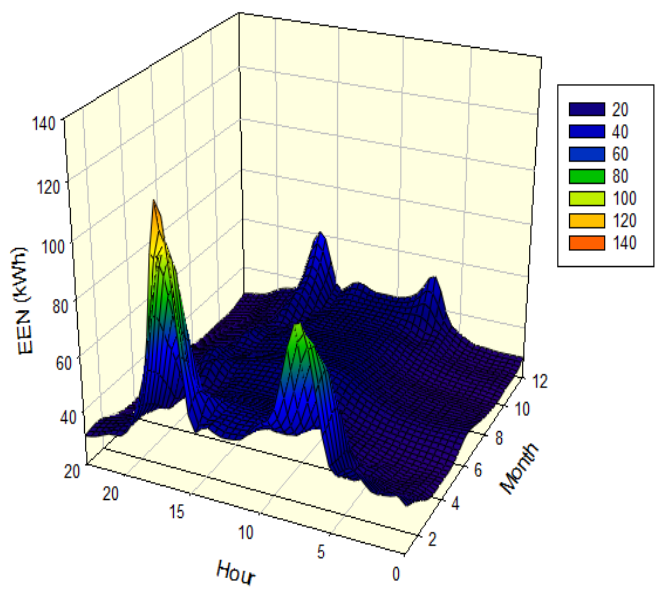

(a) Capacity release at peak load

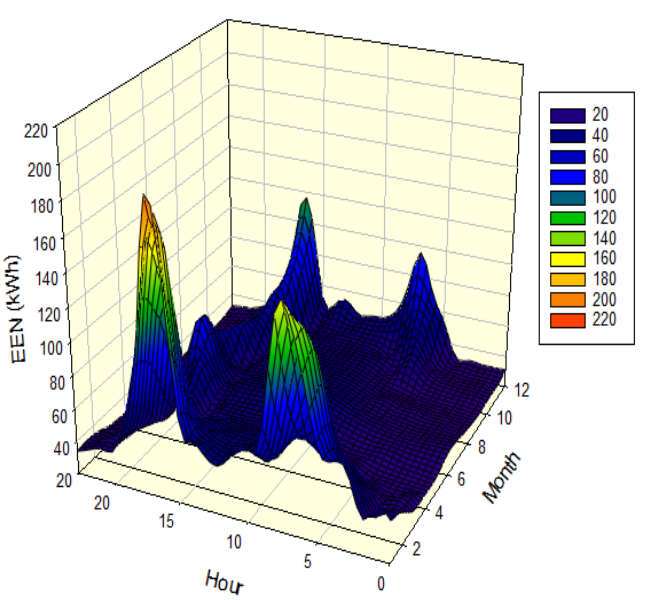

(b) Capacity release at 1.1 load growth

Figure 5.15: IEEE 34-bus capacity release with optimal size PV-DG

Moreover, an interesting observation common to all penetration and load levels considered is that the distribution test systems experienced load reduction and capacity release more during the mid-year period with the greatest amount of solar irradiance as depicted in Figure 5.4. This further confirms the fact that, in order to maximize the potential value of a DG solution in terms of incremental capacity addition and peak load relief, it is pivotal that the proposed DG generate power at proper times and levels.

However, five instances of demand increase (e.g. within 5 years) may not be sufficient to characterize the trend for EEN percentage reduction with load growth. Figures 5.16 and 5.17 represent an extrapolation of a possible load growth scenario to characterize EEN reduction with the proposed PV-DG incremental solution over 20 years. Figure 5.16 vividly shows that the optimal size frees up most of the capacity of the IEEE 13bus feeder, and therefore, contributes to the enhancement of the system reliability.

Also, as shown in Figure 5.17, a 15\% penetration can no longer support further load growth without increasing the EEN above the basecase 


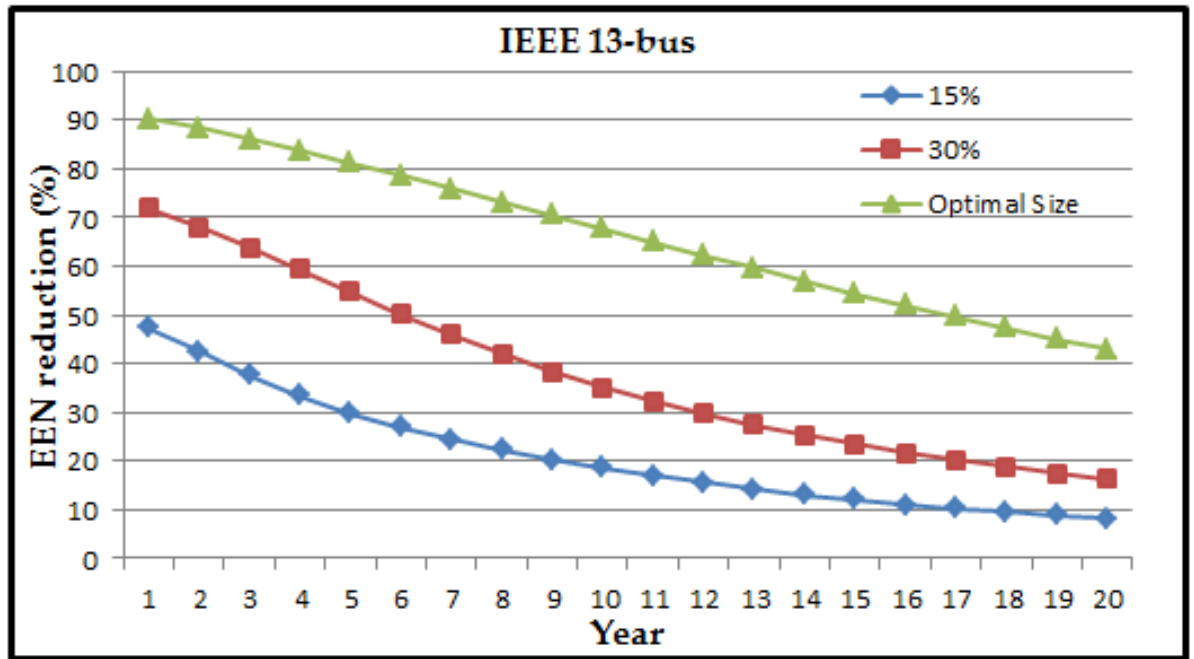

Figure 5.16: IEEE 13-bus EEN reduction with load growth for all PV-DG cases

value after the 14th year (with 2.385 growth rate) on the IEEE 34-bus system. However, $30 \%$ and optimal size application can support load growth beyond the $15 \%$ constraint.

Furthermore, as the demand increases, the rating of the PDE rises above the "Normal" and tends towards the "Emergency" rating as shown in Figure 5.1.

Results from the multiyear simulation shows this trend as depicted in Figure 5.18. For IEEE 13, as a heavily loaded feeder, the overload emergency limit rises above zero from $3.5875 \mathrm{MW}$ (i.e., 1.025 growth rate) loading and significantly increases as the load grows. This implies that some loads will be curtailed or unserved to reduce the power to a manageable level. However, with an optimal size PV-DG, the emergency limits are not violated until the load reaches 3.9375 MW (i.e., 1.125 growth rate). In the case of IEEE 34, the maximum PDE rating is not immediately violated until 2.295 MW loading (i.e., 1.275 growth rate) being a lightly loaded feeder. Also, the optimal size extended this limit till 2.52 MW load (i.e., 1.4 growth 


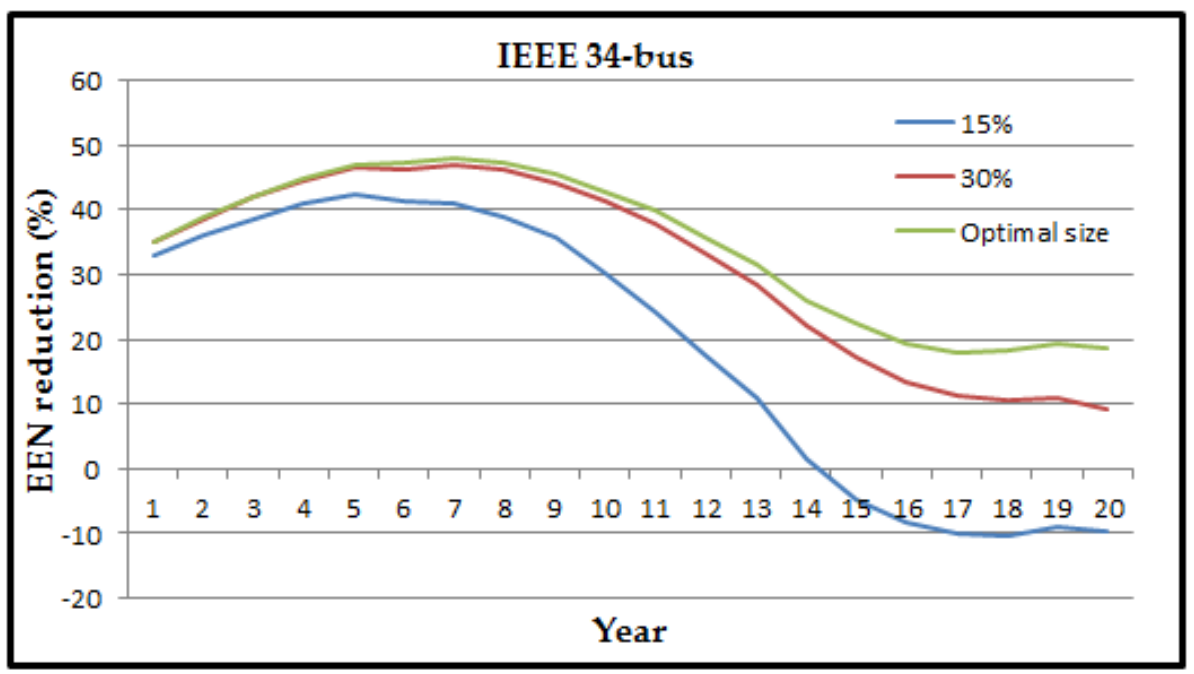

Figure 5.17: IEEE 34-bus EEN reduction with load growth for all PV-DG cases

rate). In general, the violation of the maximum limit is deferred with respect to the PV-DG penetration level.

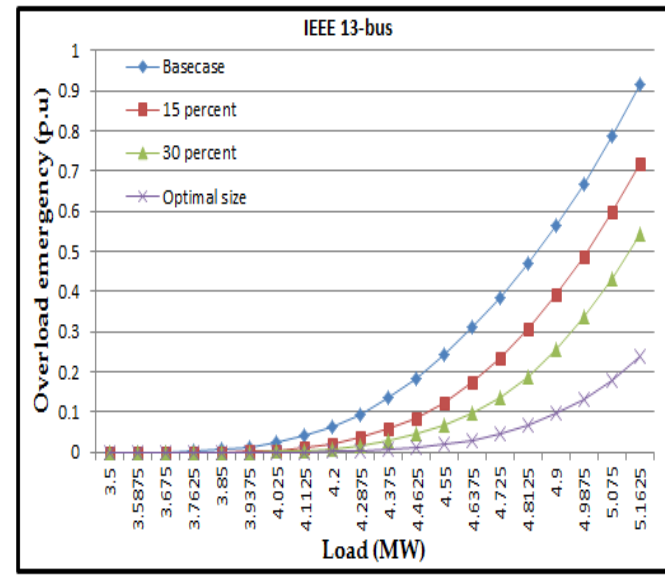

(a) Overload emergency

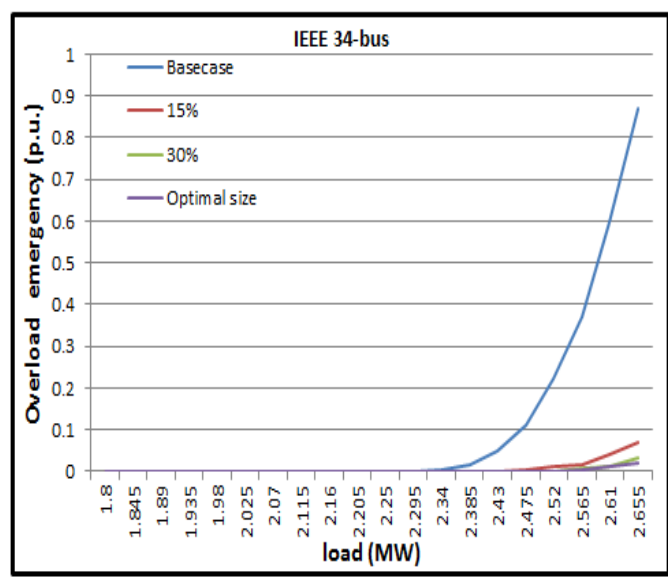

(b) Overload emergency

Figure 5.18: Basecase annual energy losses at peak load for both systems 


\subsubsection{Incremental capacity evaluation}

EEN values are used to quantify the capacity gain for each of the proposed PV-DG application in both distribution systems considered. For any DG application to add a tangible capacity to the EPS, then it must be capable of generating power during periods when the EEN characteristic is highest [145]. The incremental capacity is obtained by computing the distance between the basecase and PV-DG curves along the horizontal axis as shown in Figures 5.19 and 5.20. This curve is used by distribution system planners to determine how much load growth can be allowed in the area EPS until the EEN equals the basecase value.

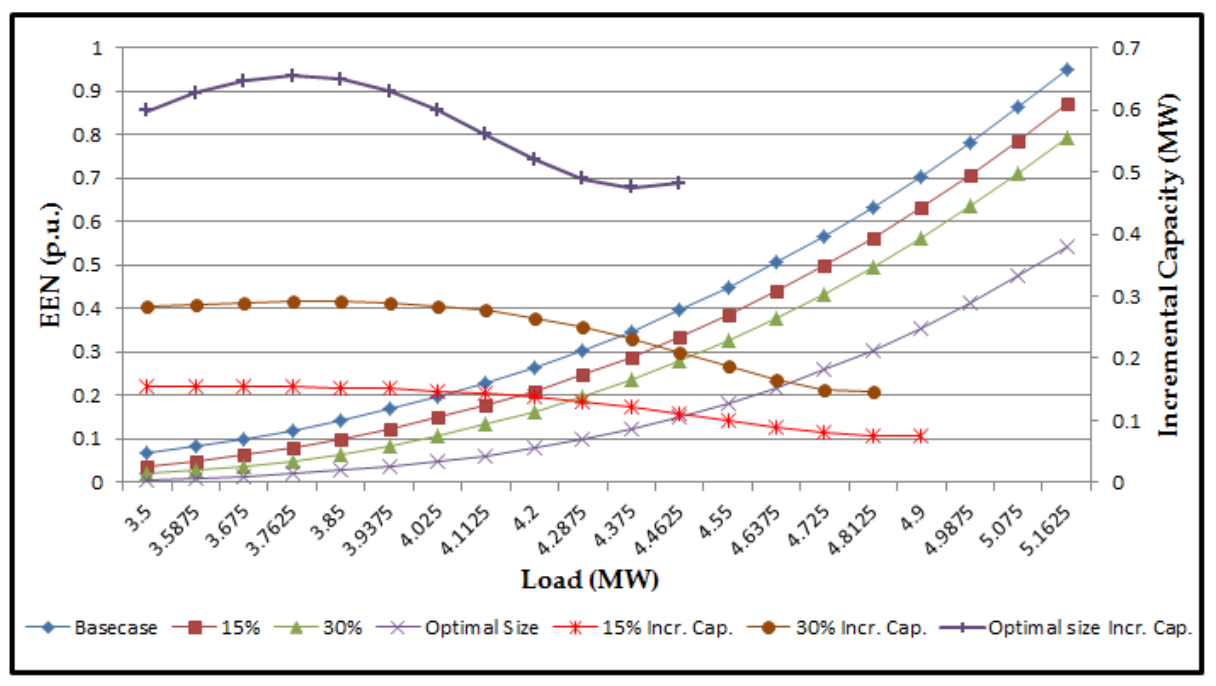

Figure 5.19: IEEE 13-bus incremental capacity computation based on EEN

For IEEE 13-bus, the incremental capacity curves show an initial increasing trend and then declines as the load grows from 3.5 MW to 5.1625 MW. In the case of optimal size application, the additional load serving capacity grows from $598 \mathrm{~kW}$ to $650 \mathrm{~kW}$ and then decreases to $481 \mathrm{~kW}$ as the demand grows.

Furthermore, the $15 \%$ penetration in the IEEE 34-bus shows a very interesting result. As the demand grows from $2.43 \mathrm{MW}$, no capacity gain 
can be attributed to this penetration level because power produced can no longer support load growth beyond this loading. However, this particular application may still enhance loss reduction, energy efficiency and demand decrease at the point of interconnection with the area EPS.

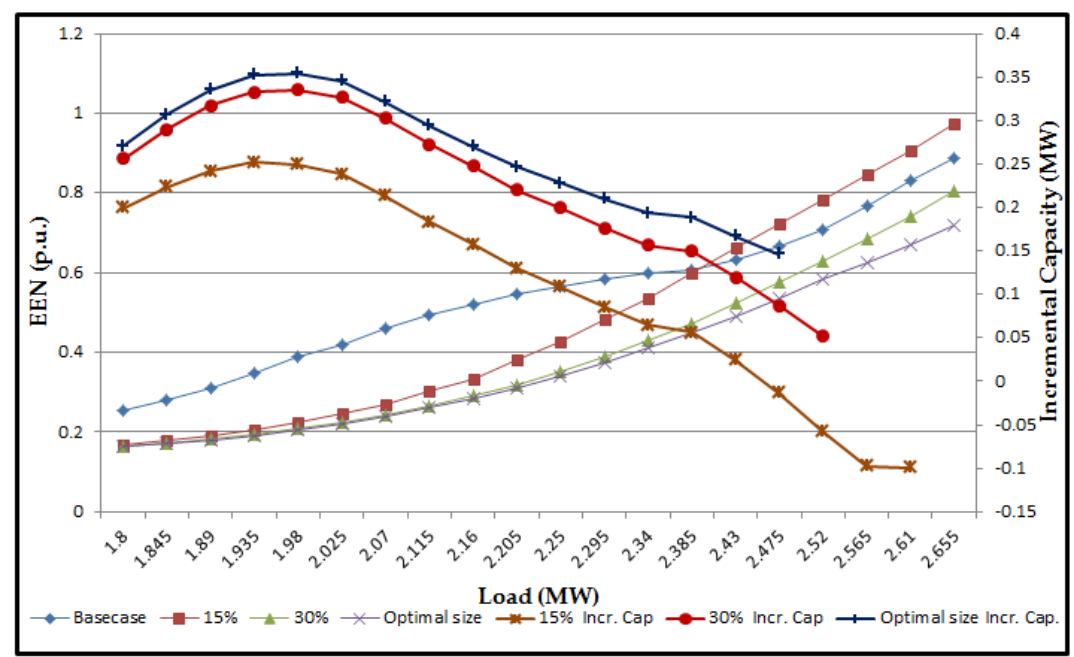

Figure 5.20: IEEE 34-bus incremental capacity computation based on EEN

In addition, as shown in Figures 5.19 and 5.20, the incremental capacity curves for both systems increase to a particular value and then decrease as load increases and constraints in other parts of the network becomes critical and start to dominate EEN computations. Also, the increasing trend shows that a large amount of capacity has been freed up in the PDE.

\subsubsection{Energy Losses}

\section{Basecase Losses}

A very pivotal plot from the initial annual simulations is the basecase energy losses for the two test systems as shown in Figure 5.21. This plot is very important because it identifies period within the year when the distribution network will most likely benefit from peak demand relief caused 
by DG installation. From the plot, the period of greatest interest would be from 10:30 am to $6 \mathrm{pm}$ at the beginning, middle and close of the year.

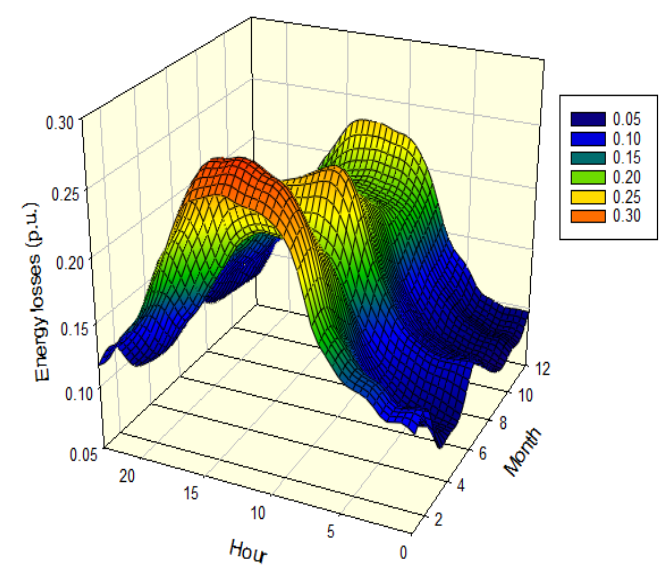

(a) IEEE 13-bus annual energy losses

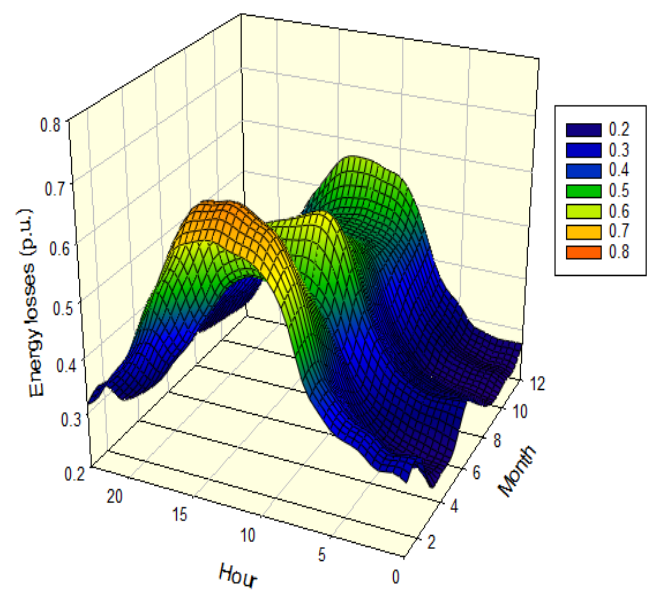

(b) IEEE 34-bus annual energy losses

Figure 5.21: Basecase annual energy losses at peak load for both systems

\section{Impact of PV-DG on losses}

In order to enhance the utility's energy efficiency, it is pivotal to ensure the reduction of energy losses especially in the distribution segment which contributes about $70 \%$ to system losses [144]. A good PV-DG application would generally lead to reduction of losses through current injections at the point of connection with the area EPS. For both feeders (as shown in Figure 5.22), the optimal size PV-DG application results in greater loss reduction than other penetration levels as the load grows.

However, all the PV-DG applications contributed to loss reduction as the demand increases indicating that the generation is not too large for the PDE in the area EPS as depicted in Figure 5.22. In addition, this analysis suggests that the PV-DG is well-located to reduce losses and serve future growth in demand for both feeders. 


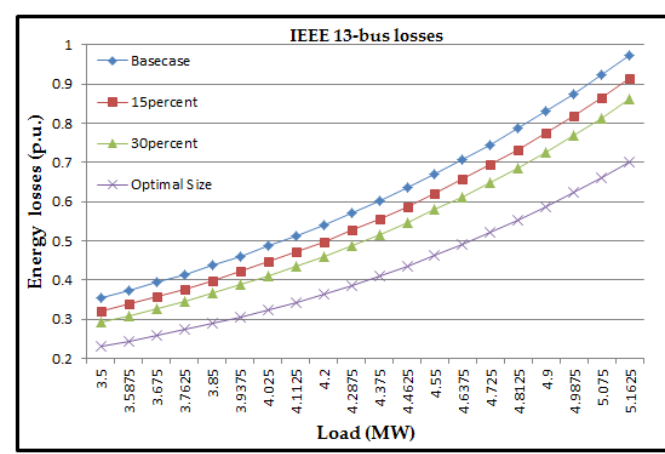

(a) IEEE 13 losses

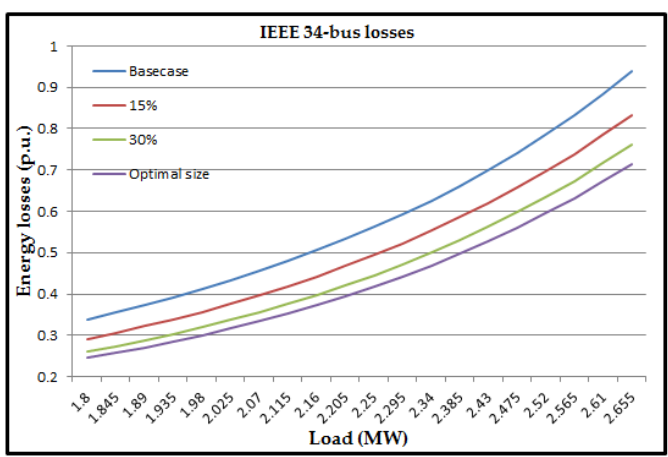

(b) IEEE 34 losses

Figure 5.22: Comparison of losses with load growth for all cases

\subsubsection{UE offset by PV-DG penetration}

The inevitable growth in peak demand increases the risk of some loads being unserved as a result of the violation of the PDE emergency limits. The deployment of PV-DG can be used to free up the PDE capacity and offset the UE with the possibility of deferring distribution investments. Figures 5.23 and 5.24 show the UE curves for all PV-DG cases in both systems.

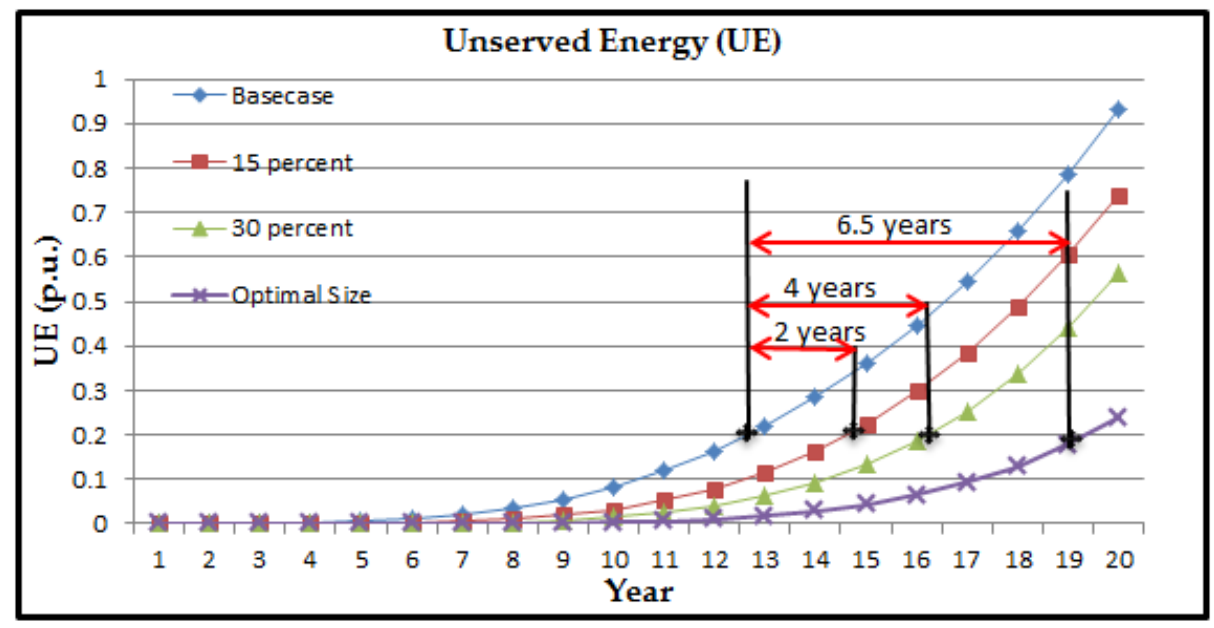

Figure 5.23: IEEE 13-bus unserved energy for all PV-DG cases

In Figure 5.23, for IEEE 13-bus, the UE does not start to rise signifi- 
cantly until year 7 when the load is projected to exceed the emergency limits and therefore, expected to be curtailed or shed to avoid equipment damage. Also, in case of the IEEE 34-bus as shown in Figure 5.24, the UE exceeds the basecase value significantly in year 6 at which time the maximum limits are violated.

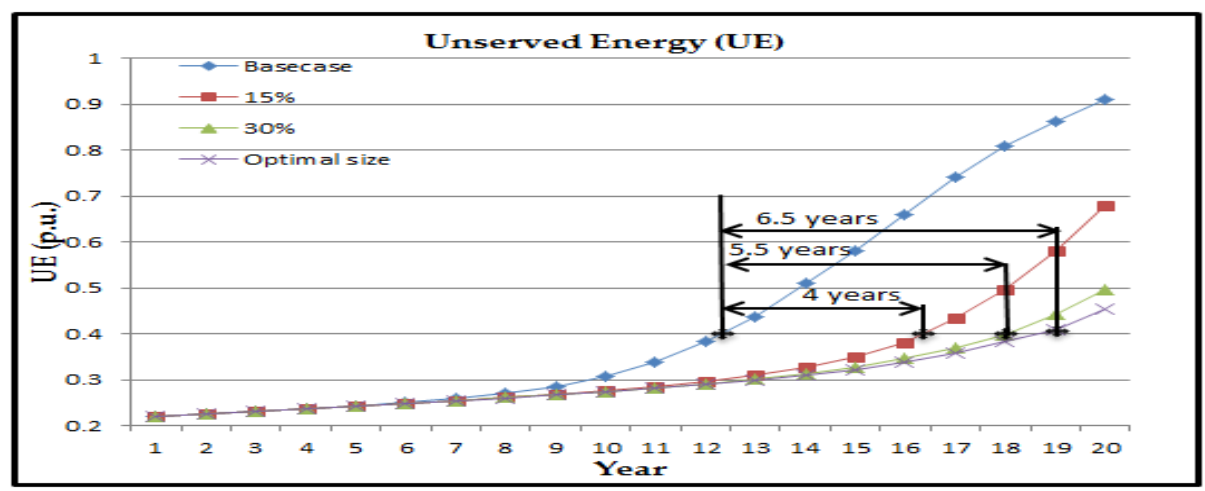

Figure 5.24: IEEE 34-bus unserved energy for all PV-DG cases

Utility companies would plan for an upgrade of the PDE prior to peak demand in year 7 (IEEE 13-bus) and 6 (IEEE 34-bus) to minimize the risk of unserve energy and the impact on grid reliability. Therefore, the issue being addressed here is how the proposed various PV-DG applications can help defer the needed system upgrade. For instance, at the same risk of basecase unserved energy of 0.2 p.u. in Figure $5.23,15 \%, 30 \%$ and optimal size PV-DG are capable of providing technical capability for 2, 4 and 6.5 years' upgrade deferral respectively. Also, for the IEEE 34-bus, at the same risk of basecase unserved energy of 0.4 p.u. in Figure 5.24, 15\%, 30\% and optimal size PV-DG are capable of providing technical capability for 4, 5.5 and 6.5 years' upgrade deferral respectively.

The benefit of deferral is obtained in terms of financial cost savings accrued from a delayed feeder construction. Also, this is premised on the condition that the inflationary increase in costs to construct a new feeder in the future is less than the utility's weighted average capital cost. In addition, this analysis becomes very critical in scenarios where the demand 
increase will outpace the construction of a new feeder, and where expansion is expensive or difficult to achieve.

\subsection{Chapter summary}

Optimally deployed utility-interactive PV systems can be used to effectively enhance the load serving capability of the power delivery system while satisfying the reliability requirements of the electricity grid. The additional load serving capacity provided by PV-DG now makes it necessary to include this generating resource in the distribution system planning. The intrinsic correlation between network capacity and reliability further gives the computation of the PV-DG incremental capacity solution a prime place in the re-engineering and design of the evolving active distribution system planning.

Two energy metrics, energy at risk (EEN) and UE, have been used for this computation and the measurement of the duration of upgrade deferral as a result of the optimal deployment of the PV-DG in the network. These energy metrics are proposed as surrogates for evaluating reliability, based on concrete limits defined by the normal and emergency ratings of the power delivery system. For a lightly loaded network such as the IEEE 34-bus, it is realised that capacity issues become critical with load growth over time when the PDE constraints could be violated. This is why a single year analysis may not be sufficient to characterize the system with the inevitable load growth and the changing landscape of the network with PV-DG penetration.

The IEEE 13 bus, as a heavily loaded network, requires the capacity of the power delivery system to be relieved as soon as possible to reduce the power to a manageable level with demand increase in the network. Apart from optimal positioning and sizing, maximizing the value attributes of PV-DG also, depends on its ability to produce power at proper times and levels. Consequently, this PV-DG application will be most suitable for dis- 
tribution networks with predominantly commercial loads due to the coincidence of demand and irradiance peaks.

\subsection{Next chapter}

The next chapter presents the impact of a grid-tied large scale plant variability on the IEEE-34 distribution network using the wavelet variability model. The effect of various power factor control schemes in a time-series power flow are investigated with a high-frequency irradiance data. 


\section{Chapter 6}

\section{Impacts of power factor control schemes in time series power flow analysis for centralized PV plants using wavelet variability model}

This chapter investigates the impact of three power factor control strategies (fixed power factor, power factor schedule and function) on the power output of a centralized utility-interactive photovoltaic (PV) plant deployed close to the feeder end and source using the wavelet variability model (WVM) at various penetration levels. The upscaling advantage from a single module and point irradiance sensor to geographic smoothing over the entire PV footprint in WVM is used to simulate effects of a grid-connected large PV system on the IEEE-34 distribution feeder. Also, this study uses a high-frequency solar irradiance data (1s) to show impacts of PV output variability on voltage regulator tap changing operations, feeder voltage, active and reactive power profiles at various penetration levels until the voltage constraint is violated. The results in this chapter have been have been peer-reviewed and published in [23] 


\subsection{Introduction}

The increasing presence of PV systems in the EPS with a global installed capacity at $303 \mathrm{GW}$ and about $75 \mathrm{GW}$ of additional capacity in 2016, calls for an interconnection study with high penetration scenarios [172]. However, the integration of PV plants as a low-carbon source of power has the propensity to cause emergent behaviours in the traditional electric power system (EPS) due to its inherent variability, location-specificity and uncertainty [11]. Moreover, the intermittency of the solar PV system as a result of factors such as cloud movements, variable constituents of the atmosphere and balance-of-system components pose a critical challenge in its integration with the EPS. Cloud-induced variations impact the grid more because of the high propensity to cause unpredictable fluctuations in power generations at short durations $(<1 \mathrm{~h})$ [173]. It is pivotal for grid operators and system planners to understand the variability of the solar resource and power produced in order to have a sufficient measure of the actual impact of such fluctuations on the EPS and subsequently, provide adequate mitigation measures. Also, geographic smoothing of solar irradiance over the PV power plant area is important in predicting PV system inherent variability with its associated integration issues [173], [174]. The evolving EPS must have the capability to withstand and respond adequately to the intrinsic fluctuations of utility-interactive PV systems without compromising grid reliability [175]. Distribution system planners are keen on knowing the effect of solar ramps on network devices to avoid financial losses and other forms of penalties for service delivery violations [175].

The extant literature presents integration study with irradiance data from a single point sensor without considering the entire foot print of a PV system and the impact of cloud-induced fluctuations. However, Lave et al. [173], [174] developed and validated the wavelet variability model (WVM) to be used for an accurate simulation of the variability of solar 
irradiance and the actual PV plant power output considering the entire footprint with a single point sensor. This model quantifies the aggregate variability reduction (VR) as a result of the upscale from a single PV module and point sensor to the entire PV footprint. Also, the reduction in variability is due to the decrease in instantaneous crossing of cloud edges over a single PV module and the geographic diversity of units within a PV plant [173], [174]. Importantly, utilities and authorities having jurisdiction over grid (AHJs) can use this model to understand the effect of solar power fluctuations and the advantages of geographic smoothing over the entire PV plant footprint [176].

Ana et al. [175] used the WVM in modeling irradiance smoothing for large PV power plants, and showed that the daily VR index is a function of the daily location-dependent cloud speed scaling coefficient. In another study, Lave et al. [18] presented the impact of solar variability on the number of tap operations of the voltage regulator. Results showed an increase in tap operations over the basecase for all PV deployment scenarios considered.

However, very few studies have been able to use high-frequency data $(<30 \mathrm{~s})$ and geographic smoothing to model the distribution feeder impacts considering increasing PV penetration in the evolving EPS. Paravalos et al. [177] presented an optimal design of PV plants with a 1-minaverage irradiance data used to estimate a PV system performance. Mokryani et al. [178] used an average hourly solar irradiance to model uncertainties of loads and PV systems in the distribution network. Hung et al. [41] used an hourly historical data to determine PV penetration for distribution networks with time-varying load demand models. A high resolution data is critical for a thorough impact study in order to fully characterize for example, the tap changer operations which has very short time constants (e.g., $1 \mathrm{~s}, 10 \mathrm{~s}$, etc.) [18].

Therefore, in order to develop realistic projections of the impact of PV output variability, there is a critical need to model with a high-frequency 
solar irradiance data on seconds time-scale and perform geographic smoothing over the plant footprint. The use of historical average hourly data in simulating the output of the PV plant has made it difficult for the utility and AHJs to assess the true impact and provide the necessary mitigation alternatives.

Also, the review of relevant extant literature on PV integration study shows that the impact of PV plant layout and cloud speed on the output power of the PV system have not been considered, which are pivotal because the output of such systems determines their actual impacts on the operations of the EPS. The WVM takes into account all these factors to determine the actual PV output and therefore, provide true analysis of the effects of PV output intermittency on the operations of the grid.

The contribution of this chapter is to model impacts of variability and locational value of centralized PV systems in a distribution network with a high-frequency solar irradiance data (1 s) and geographic smoothing using the WVM. Here, a duty cycle dispatch simulation is used to study the true effects of solar intermittency at various penetration levels, which is an important step in incorporating such a variable resource in the EPS operations. Further, this study uses the WVM to estimate the output of a central PV plant operating with three power factor control strategies (power factor function, power factor schedule and fixed power factor) at various penetration levels until the voltage constraint is violated.

Analysis of two centralized PV deployment scenarios such as a single PV system close to the end and source of the IEEE-34 bus feeder are presented at different penetration levels. Impacts on the feeder active and reactive power profiles, voltage regulator tap changing operations, maximum and minimum feeder voltages are presented. Also, two types of customers (i.e., commercial and residential) with different time-varying load patterns for an entire year (8760 hours) are incorporated in the test feeder. 


\subsection{Centralized solar PV system}

The availability of incentives in the form cheap capital and competitive tenders (less than 50USD/MWh) are currently driving the deployment of centralized PV plants at a higher rate over small-scale rooftop integration. In 2015, these utility-scale plants represented over $60 \%$ of the market which was majorly driven by China, United States of America and growing PV markets around the world [179]. Solar PV systems deployed as centralized power generations are large-scale installations ( $>1 \mathrm{MW})$ sited at locations of prime resource availability and usually in remote locations. Such systems enable a robust DG planning technique due to its flexibility in allowing diverse integration locations and utility load peak reduction [180].

Also, centralized photovoltaic systems are large three phase units which could be connected on existing electricity infrastructure as shown in Figure $6.1[10]$.

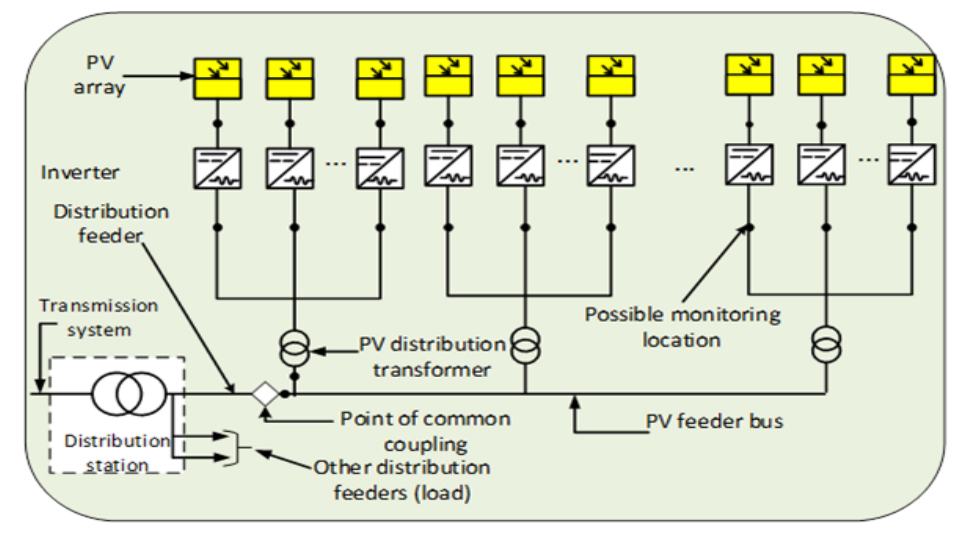

Figure 6.1: Distribution-interactive utility-scale PV system

As shown above, PV distribution transformers are step-up transformers (liquid immersed or cast resin) used to increase the voltage level of the PV output power to a point suitable for grid interconnection [10]. However, drawbacks of centralized PV plants interconnection include the need for large land areas (approximately 1-10's of sq. km) without shading and 
to construct new transmission lines especially when located far away from the current transmission network [180]. The increasing number of requests to interconnect such systems with the conventional EPS coupled with the variability of the resource places an enormous pressure on the utility to provide screening methodology and to differentiate deployment scenarios with minimal or adverse impacts on the grid.

\subsection{Solar PV modeling}

The WVM is used to simulate the output power of the PV system with the given solar irradiance data obtained from [162]. The model takes into consideration the PV system footprint and density, irradiance data 1 s resolution) from a single point sensor and a cloud speed scaling coefficient to model the smoothed irradiance over the entire plant area [5], [174], [175].

The WVM procedure is outlined as follows [173], [174], [175]:

- A wavelet transform is used to decompose the given irradiance timeseries from a single point sensor into wavelet modes $w_{\bar{t}}(t)$ at different times scales $\bar{t}$.

- For centralized PV plant considered in this study, distances, $D_{j, k}$, between every pair of PV units in a small container (or sites) is estimated, where $j$ and $k$ represent sites varying from 1 to $N$.

- The correlations between sites is calculated as:

$$
\rho\left(D_{j, k, \bar{t}}\right)=\exp \left\{-\frac{1}{A} \frac{D_{j, k}}{\bar{t}}\right\}
$$

where $A$ represents the cloud-speed scaling coefficient.

- Variability reduction $(V R)$ at each timescale $\bar{t}$ is calculated as:

$$
V R(\bar{t})=\frac{N^{2}}{\sum_{j=1}^{N} \sum_{k=1}^{N} \rho\left(D_{j, k, \bar{t}}\right)}
$$


- The simulated wavelet modes of the entire PV power plant, $w_{\bar{t}, s i m}(t)$, is given as:

$$
w_{\bar{t}, \operatorname{sim}}(t)=\frac{w_{\bar{t}}(t)}{\sqrt{V R(\bar{t})}}
$$

- An inverse wavelet transform is applied in order to form the simulated clear-sky index of area-averaged solar irradiance over the entire power plant footprint, $G_{G, s i m}(t)$, calculated as:

$$
G_{G, \operatorname{sim}}(t)=\sum_{m=1}^{12} w_{\bar{t}=2^{m}, \operatorname{sim}}(t)
$$

- The power output is then obtained by multiplying $G_{G, s i m}(t)$ by a clear-sky power output model, which considers the plant's capacity, a derating factor (e.g., due to temperature) and plane of array irradiance clear-sky model.

For the location-dependent coefficient, A value of $10 \mathrm{~m} / \mathrm{s}$ is assumed for an inland area such as Arizona [174] where the IEEE-34 bus feeder is located. The WVM is applied on the input irradiance data to obtain the WVM smoothed irradiance as shown in Figure 6.2.

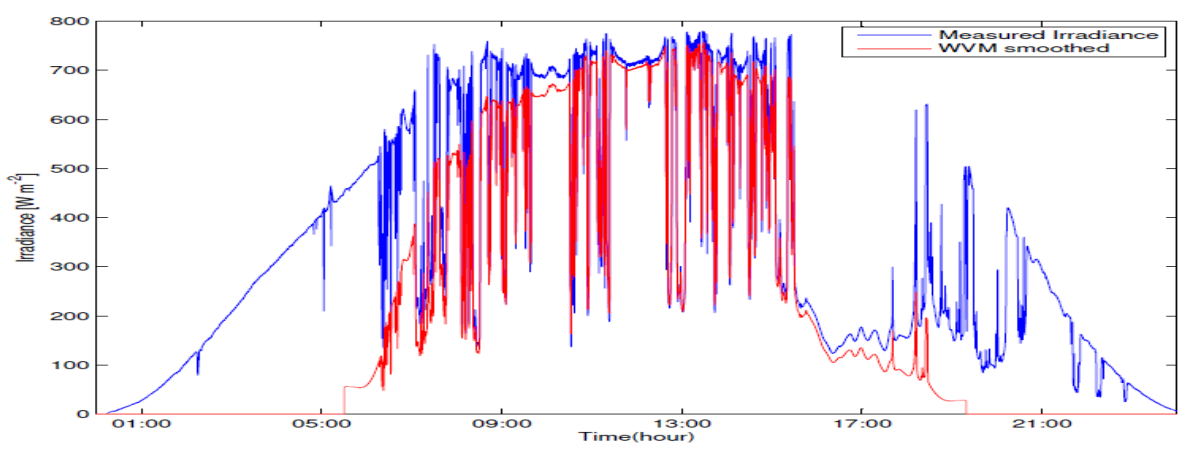

Figure 6.2: WVM smoothed irradiance for the input irradiance

This represents the average irradiance over the entire PV plant footprint and also, maintains the time stamps of the input irradiance. 
The VR is estimated for $15 \%, 30 \%$ and $45 \%$ penetration levels of the peak active power demand in the IEEE-34 bus feeder as shown in Figure 6.3. A high value of $V R$ at a particular timescale shows a decrease in the total variability.

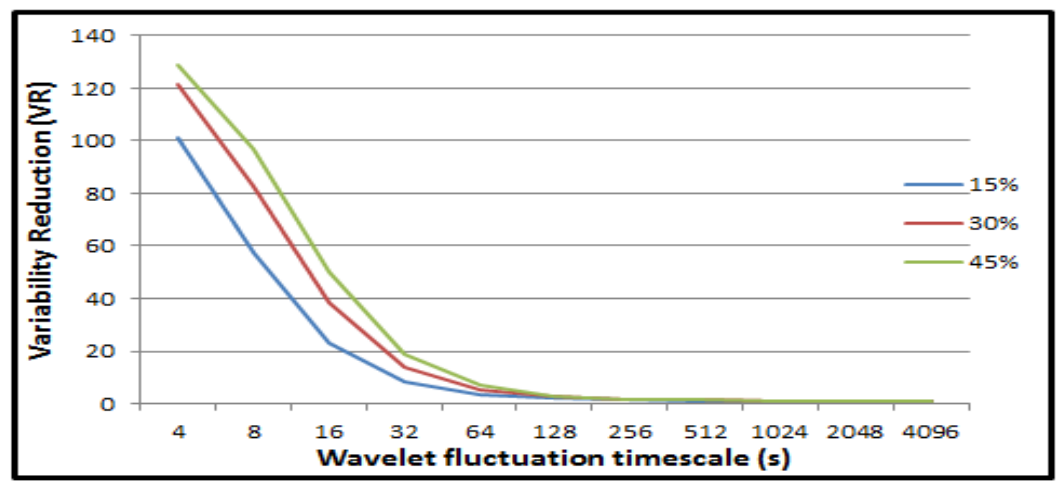

Figure 6.3: Variability reduction at different timescales

Also, from Figure 6.3, the greater the PV plant size, the greater the $V R$, which invariably indicates a decrease in variability with increasing size or penetration level. In addition, with longer time scales ( $>60 \mathrm{~s}$ ) the variability reduces significantly to about 1 and the smoothing becomes very small. Therefore, at longer time scales such as the hourly duration, the correlation between PV units (or sites) becomes higher which often leads to significant changes in the aggregate output power over an hour [173], [181].

\subsection{Power factor control strategies}

For high levels of PV deployments on the distribution system, it is important to maintain the feeder voltage profile within the range of $0.95-1.05$ p.u. recommended by IEEE Std 1547 [30]. The impedance between the closest voltage regulating device and the PV system can cause a voltage rise at the point of common coupling (PCC) as PV power output increases [182]. Therefore, the PV output power factor can be adjusted to avoid the 
possibility of voltage violations. In this study, three control strategies for adjusting the PV output power factor are considered. They include the power factor schedule, power factor function and fixed (unity) power factor.

\section{Power factor schedule}

This strategy controls the power factor $(\mathrm{PF})$ during day time as shown in Figure 6.4. This helps the distribution system engineers to adjust the impact on voltage levels at particular times of the day by modifying the PV inverter PF to output Vars for voltage support [5], [182].

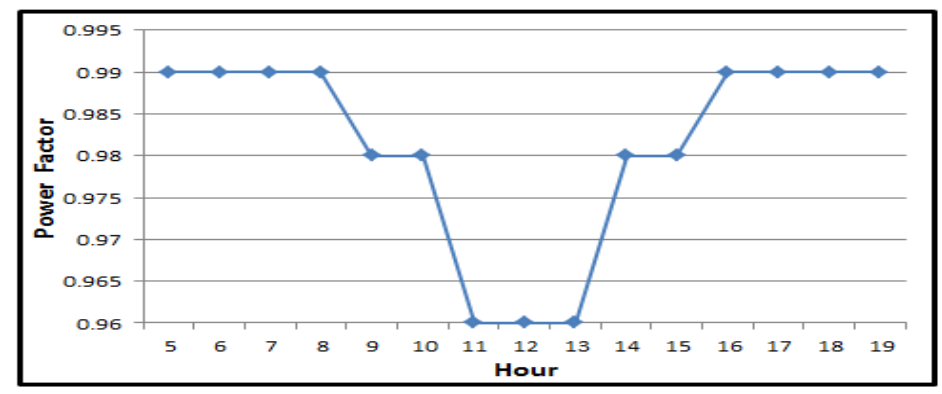

Figure 6.4: A typical power factor schedule

The schedule in Figure 6.4 shows a typical decrease in PF during periods of peak solar generation, which happens usually in the middle of the day.

\section{Power factor function}

This strategy controls the PF as a function of power output from PV as depicted in Figure 6.5, which can be used to maintain the system voltage [5], [182].

Also, Figure 6.5 shows that for lower PV output, the PF is closer to unity to provide voltage support and gradually decreases as the output increases. 


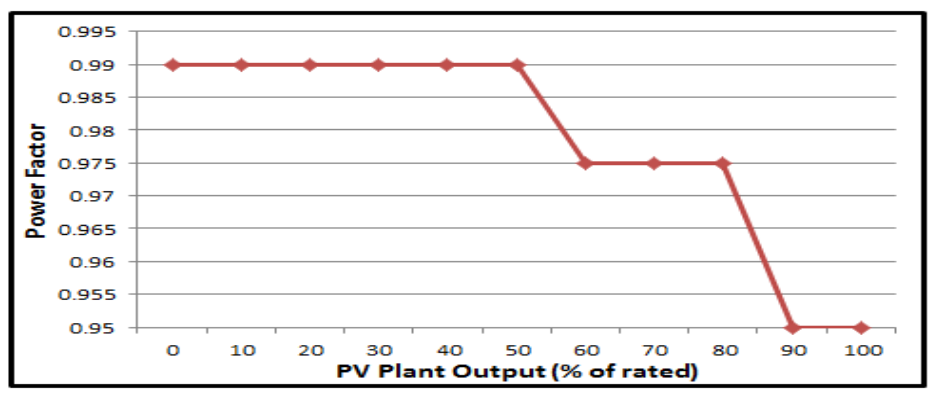

Figure 6.5: A typical power factor as a function of PV output

For example, using the WVM, the real and reactive output power produced from the PV plant at different penetration levels, are depicted in Figures 6.6 and 6.7 .

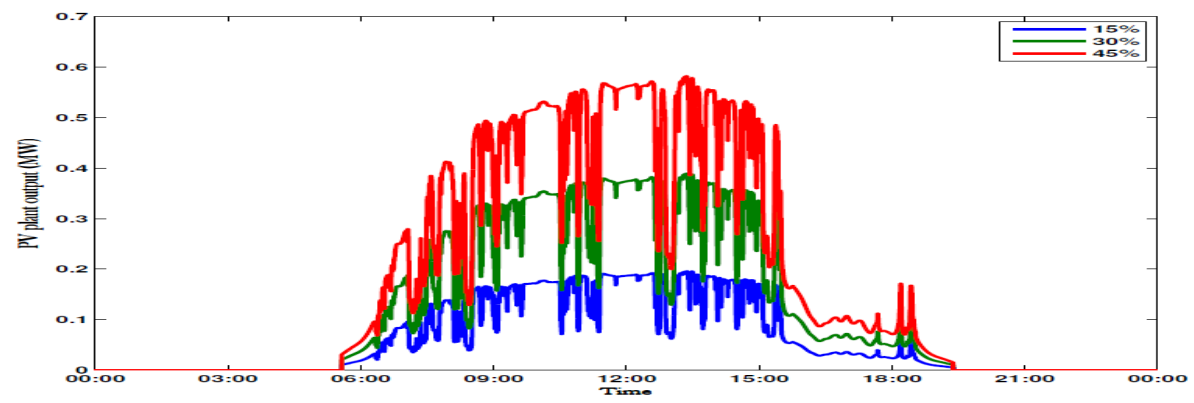

Figure 6.6: Active power produced at various $P_{L}$ modeled using WVM

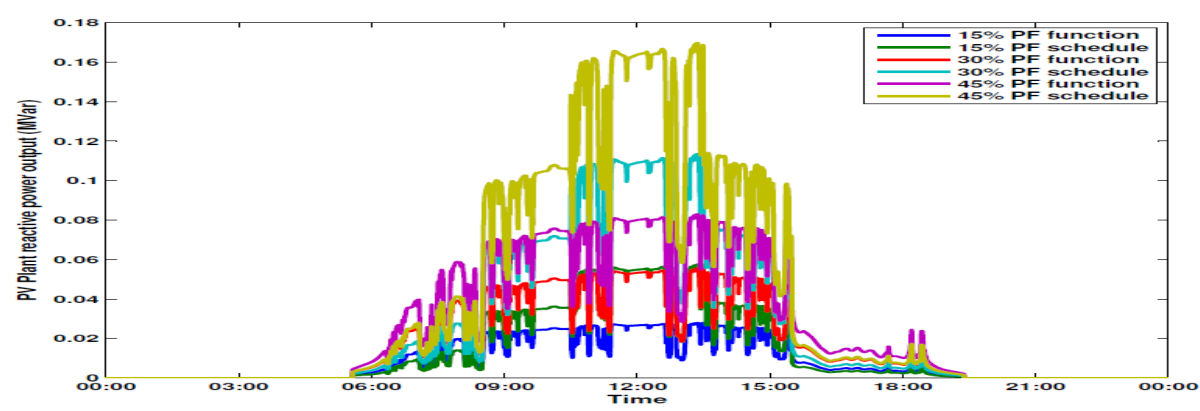

Figure 6.7: Reactive power produced at various $P_{L}$ modeled using WVM

Figure 6.6 depicts the maximum active power generation from the PV 
plant during peak sun hours (PSH) which is proportional to the penetration level. Figure 6.7 further shows the capability of PF function and schedule control schemes to generate reactive power especially during the PSH for providing voltage support for the distribution system.

\subsection{Case study}

The modified IEEE-34 distribution test feeder is used as a test case with a total load of $1769 \mathrm{~kW}$ and $1044 \mathrm{kVAr}$ [171] and following the time-varying load profiles as shown in Figure 6.8. The system's load demands obtained from [162] follow different normalized annual load patterns (i.e., commercial and residential) with a peak of 1 p.u. The commercial and residential load factors are 0.7 and 0.4 respectively. The IEEE 34-bus system as shown

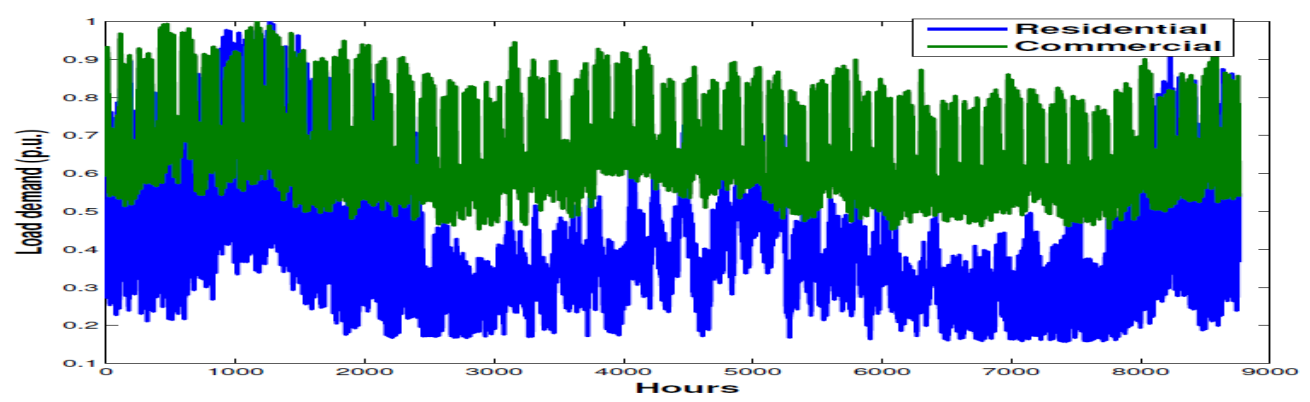

Figure 6.8: Annual normalized demand profile for various customers

in Figure 6.9, with spot and distributed loads, is a long lightly loaded feeder located in Arizona with two different operating voltages of 24.9 $\mathrm{kV}$ and $4.16 \mathrm{kV}$ [141]. The operating voltage constraint is from 0.95 to 1.05 p.u.

The sensitivity analysis (presented in Algorithm 1) used for the optimal siting showed that highest ranked buses are located towards the end of the feeder which is prone to voltage drop. However, centralized PV systems depend on solar resource availability and therefore, require the consideration of diverse resource locations on the distribution feeder. Bus 
812 ranked higher within node clusters close to the feeder source with VSI and LSI values of 0.00287 and 0.0755 respectively. Also, Bus 836 ranked higher within node clusters close to the end of the feeder with VSI and LSI values of 0.00489 and 0.2216 respectively.

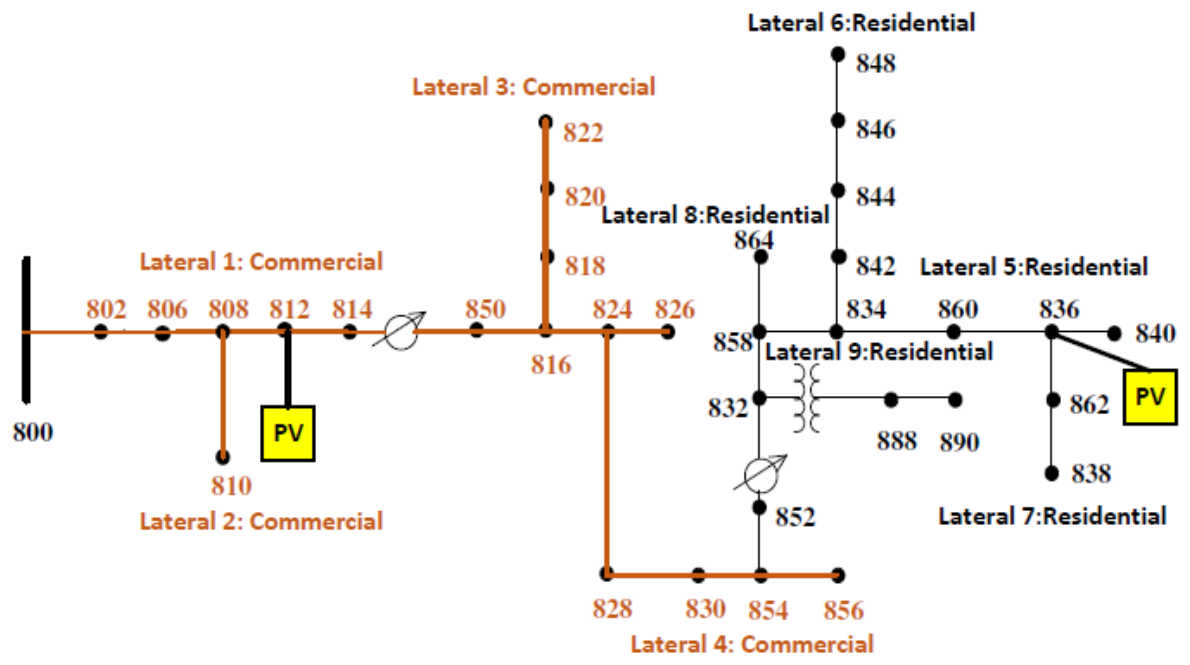

Figure 6.9: Modified IEEE 34-bus test feeder. The modification refers to the presence of PV-DG

Also, Figure 6.10 shows a satellite image of the test system with the location of the substation, voltage regulator and fixed capacitor. This is used to locate the PV system directly on the Google map and layout of the feeder, which enables smoothing of the PV variability by applying the WVM. This captures the locational value by siting the PV plant in its actual location on the circuit.

The OpenDSS uses coordinates to link each bus to its respective $X$ and Y coordinates. The GridPV toolbox is then used to map the IEEE-34 bus on the Google map (as shown in Figure 6.10) through a function that converts bus coordinates to GPS coordinates. With these coordinates, the geographic information system (GIS) is used to provide visualization of the circuit lines. The API for Google maps enables MATLAB to interact and 
download maps with data for a particular location and including elevation [13], [183]. Using the OpenDSS, Matlab coupled with GridPV, a high resolution time-series power flow analysis is carried out. Also, in this study, a single PV system sited close to the feeder source (FS) and end (FE) using Algorithm 1 are the two scenarios considered.

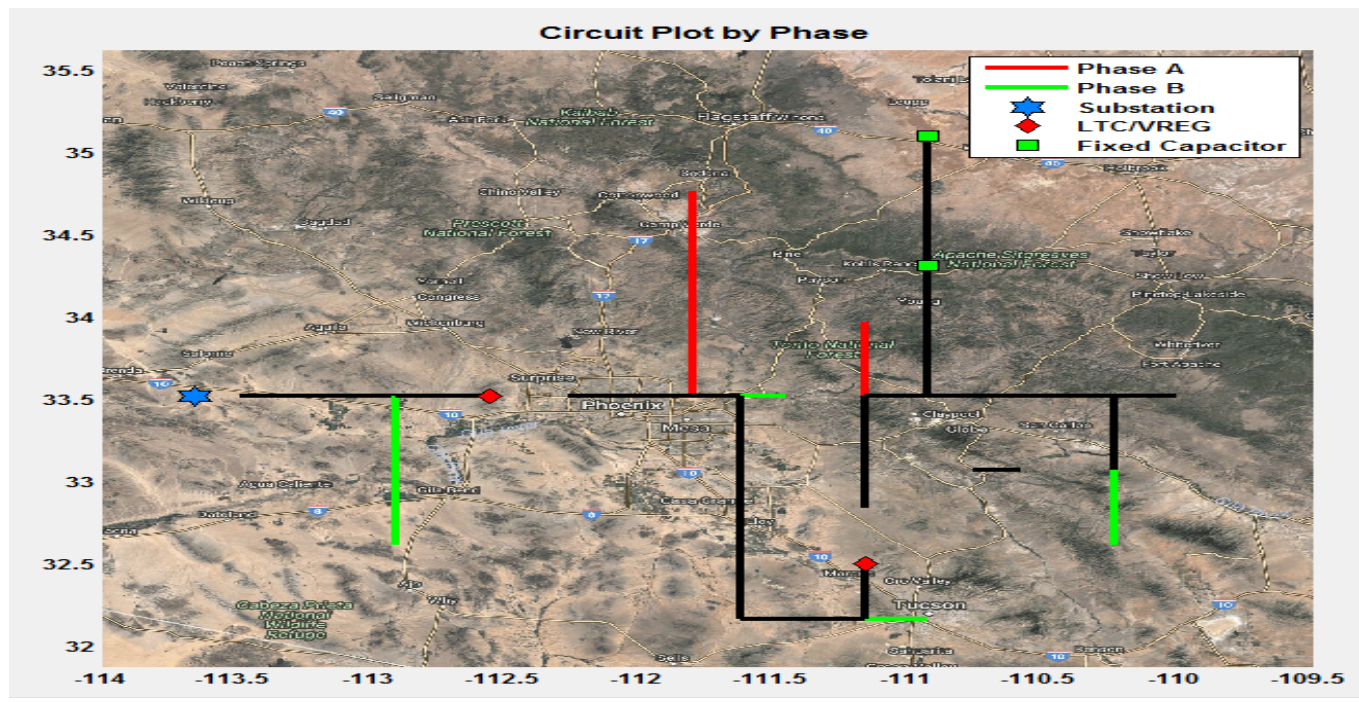

Figure 6.10: Satellite image of IEEE-34 bus topology in Phoenix, Arizona (black line is for three phase lines used in connecting centralized PV systems)

\subsubsection{Feeder voltage impacts with PF control strategies}

\section{Maximum and minimum feeder voltages}

This section presents the maximum and minimum voltages at any location on the feeder, showing the range of voltages for the basecase and a PV system deployed close to the feeder end (FE) and source (FS). This voltage analysis helps the utility to identify spots on the network with the highest and lowest voltages in order to determine if further interconnection study is required. 
As shown in Figure 6.11, for a single PV system deployed close to the feeder end, the maximum voltage occurred at hour 78.5667 on the simulation hour during periods of solar irradiance for unity PF strategy. Figure

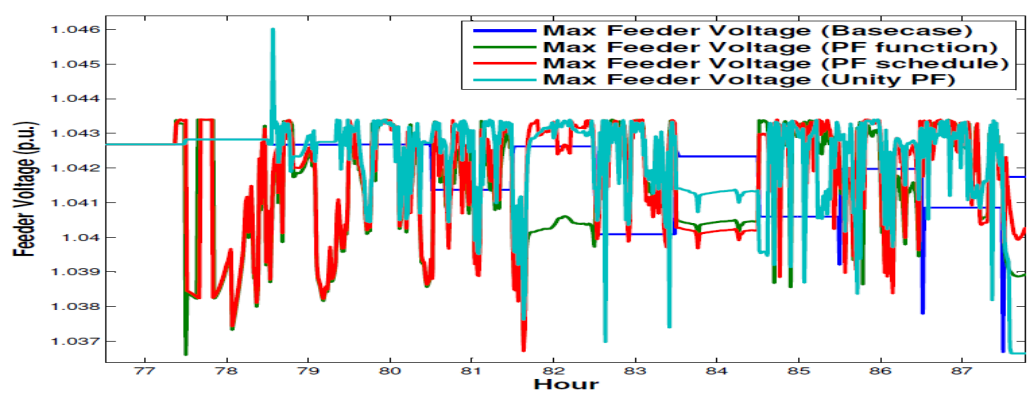

Figure 6.11: Maximum voltage with PV close to feeder end

6.12 shows that maximum voltage occurred at hour 77.83 on the simulation hour for the PV system installed close to the feeder source operating with PF schedule. Also, Figures 6.13 and 6.14 depict minimum feeder volt-

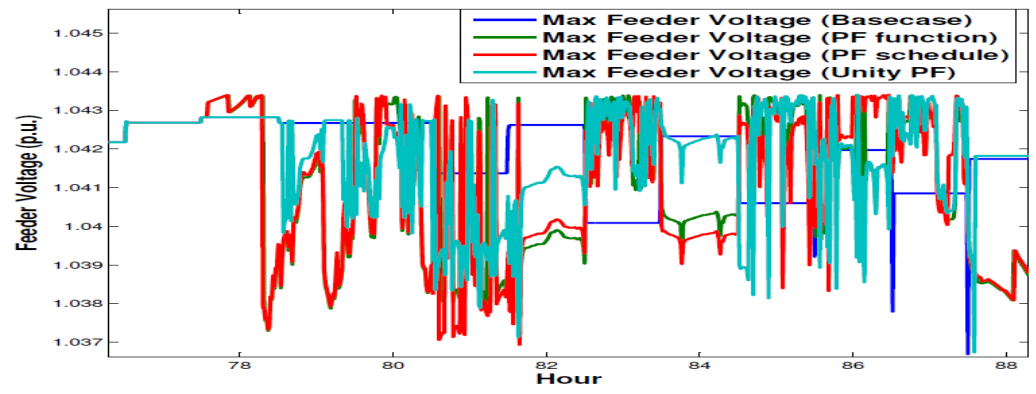

Figure 6.12: Maximum voltage with PV close to feeder source

ages for both cases of PV deployments. Figure 6.13 shows an improved minimum voltage profile for the PV unit close to the feeder source when compared with the system at far end of the feeder as depicted in Figure 6.14 .

Also, at the feeder end, the unity PF strategy resulted in voltage spikes over the other strategies for majority of the simulation hour as shown in Figure 6.14. 


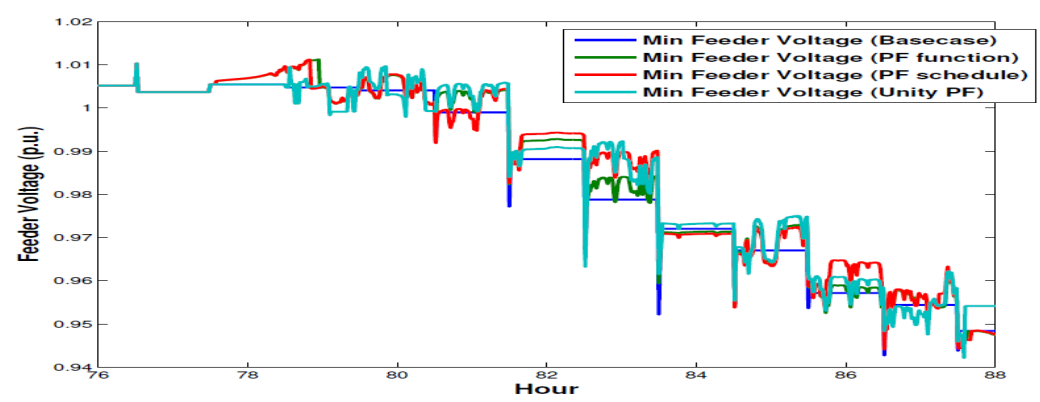

Figure 6.13: Minimum voltage with PV close to feeder source

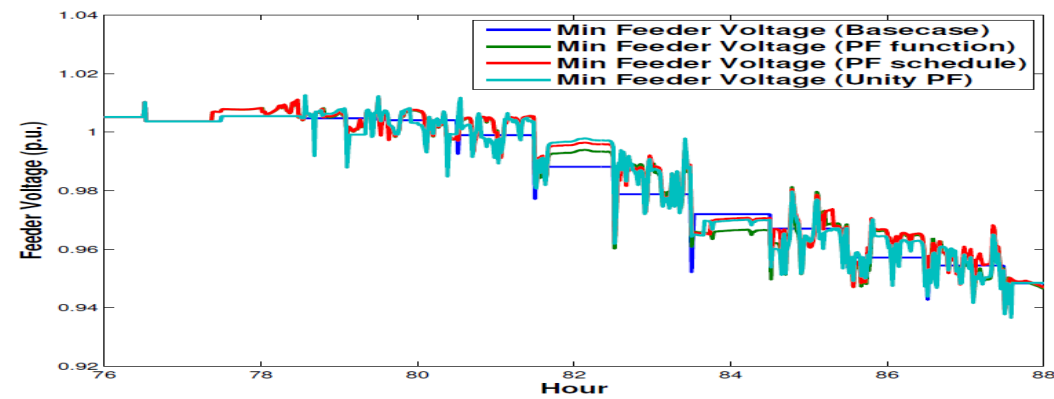

Figure 6.14: Minimum voltage with PV close to feeder end

\section{Voltage profile impact with PF control strategies}

In order to further demonstrate the impact of various PF control strategies, Figure 6.15 shows the basecase voltage profile which depicts a voltage drop as the distance from the substation increases. However, with the interconnection of PV close to the FE and FS, the voltage profile is enhanced as the penetration level increases for all PF control mechanisms.

The result reveals that the PV system deployed close to the FE caused the most voltage profile enhancement when compared to the one near the FS as shown in Figures 6.16 and 6.17. This is because for most distribution feeders, the system is electrically weaker at the far end and it takes less PV power injection to increase the voltage than it would for PV installed close the substation [55].

Furthermore, PF function and schedule strategies provided more head- 


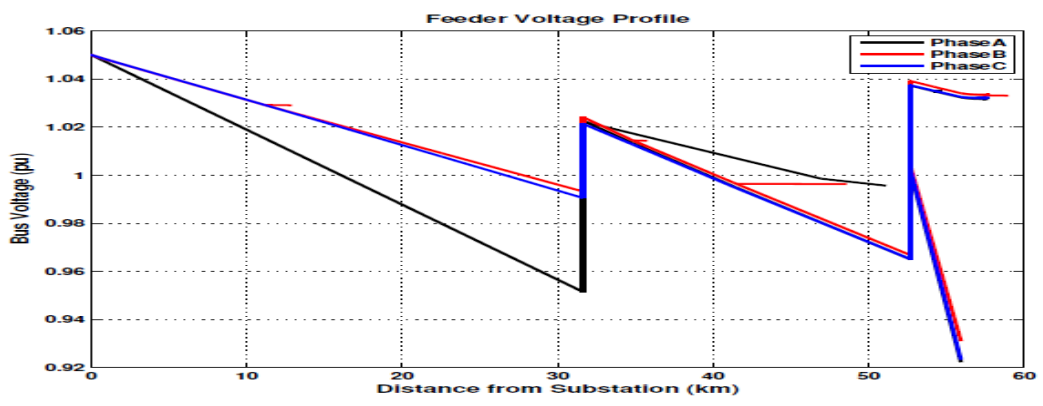

Figure 6.15: Feeder voltage profile with no PV system

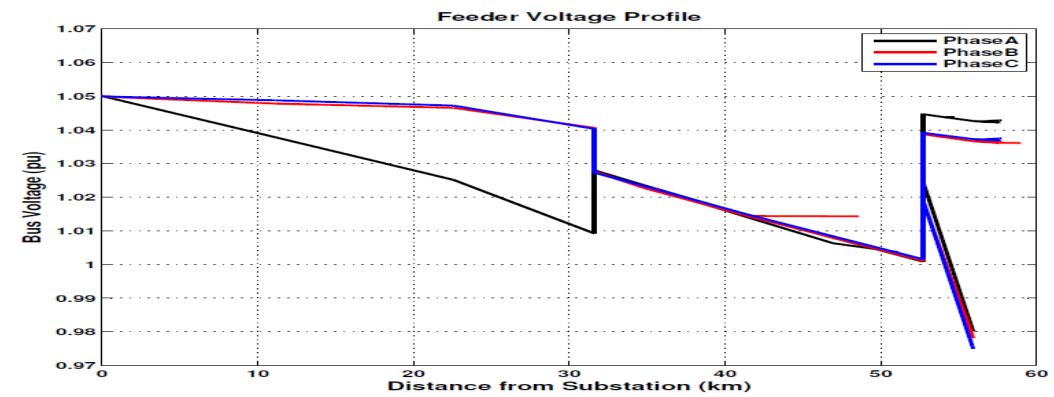

Figure 6.16: Feeder voltage profile with PV at unity PF close to the FS

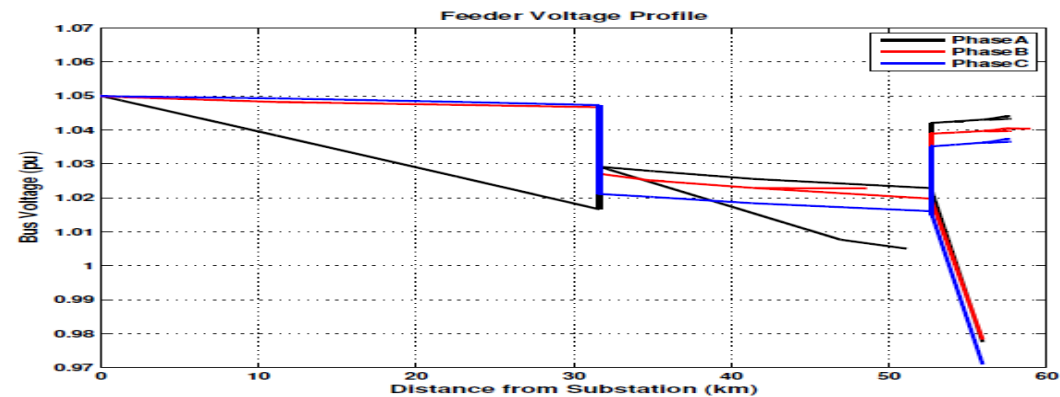

Figure 6.17: Feeder voltage profile with PV at unity PF close to the FE 
room than the unity PF as shown in Figure 6.18. Headroom refers to the difference between the feeder voltage and maximum allowable voltage [55].

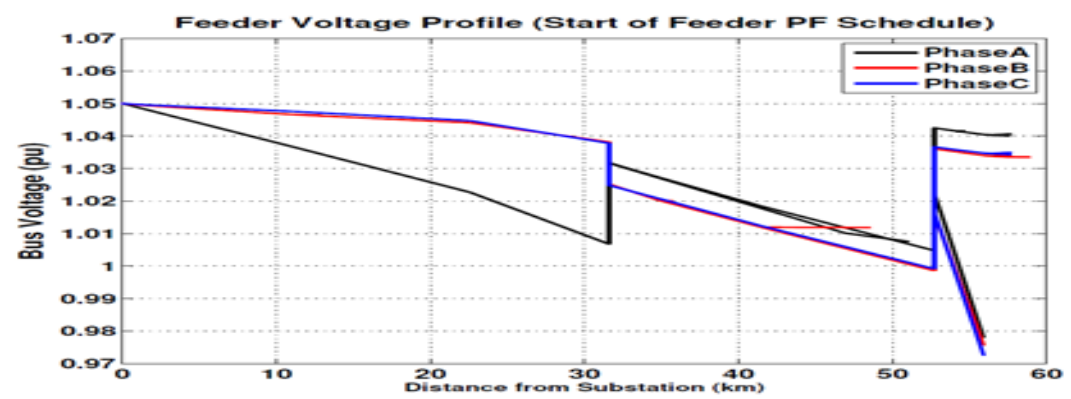

Figure 6.18: Feeder voltage profile with PF schedule PV close to the FS

\subsubsection{Impacts of variability on the voltage regulator/OLTC}

For grid operators, it is important to know the interaction between the inverter operation, on-load tap changer (OLTC), PV penetration and various deployment scenarios [13]. Importantly, a significant increase in OLTC operations could lead to increase in degradation rate and maintenance routine on voltage control devices [182]. The impact on the two LTC on the test systems deployed close to the FS (R1) and FE (R2) as shown in Figures 6.9 and 6.10 are presented.

\section{Tap operations during the sample week}

Figure 6.19 depicts the cumulative tap change operations in the sample week for R1 and R2 LTC at the FS and FE respectively with no PV system installed in the distribution network.

Results show an increase in tap changer operations as the PV penetration level increases, which is comparable to the result in [18]. An increase in tap operations for a PV system operating at unity PF with $45 \%$ penetration level close to the feeder end is shown in Figure 6.20. 


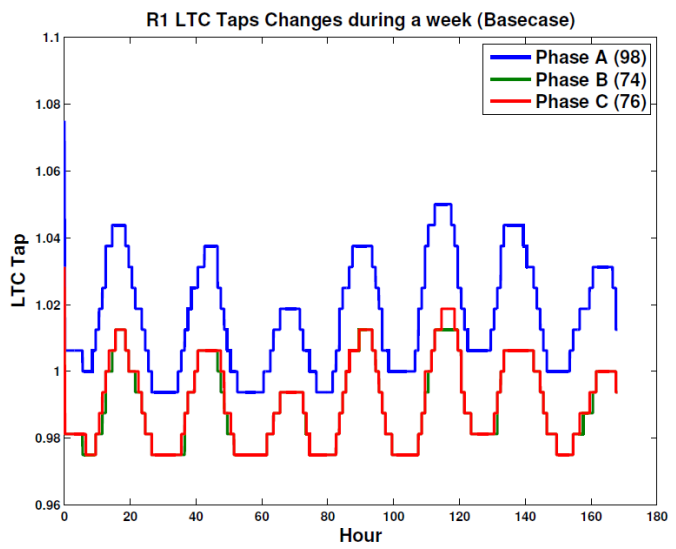

(a) R1 LTC operations

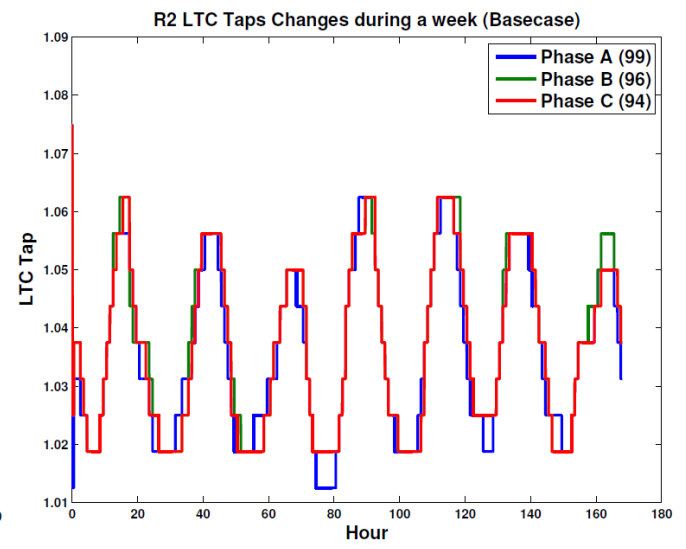

(b) R2 LTC operations

Figure 6.19: R1 and R2 LTC operations in the sample week (Basecase)

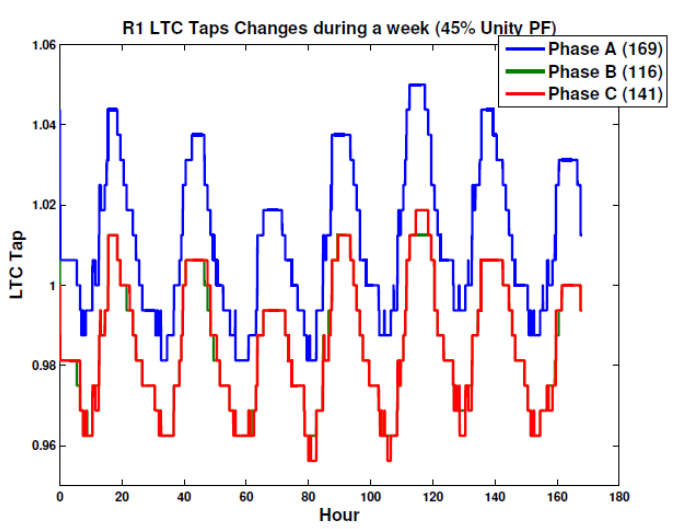

(a) R1 LTC operations for a PV at FE

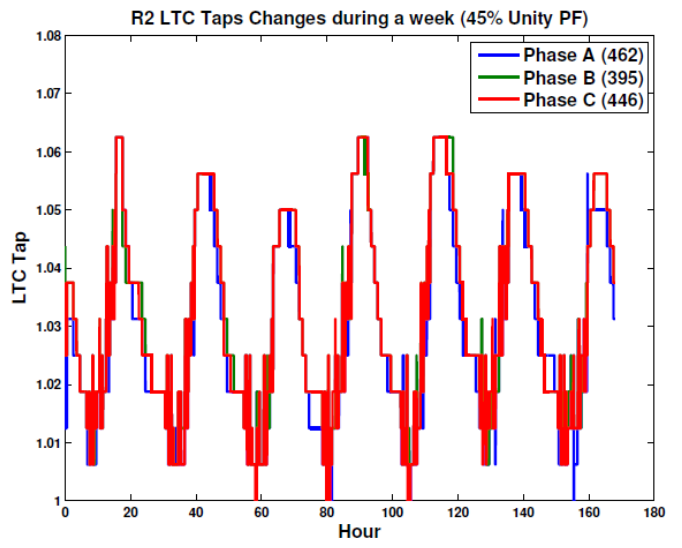

(b) R2 LTC operations for a PV at FE

Figure 6.20: R1 and R2 LTC operations in the sample week with PV plant at FE 
However, a summary of the cumulative tap operations for various PF control strategies and PV systems deployed close to the feeder source and end is presented in Figures 6.21 and 6.22.

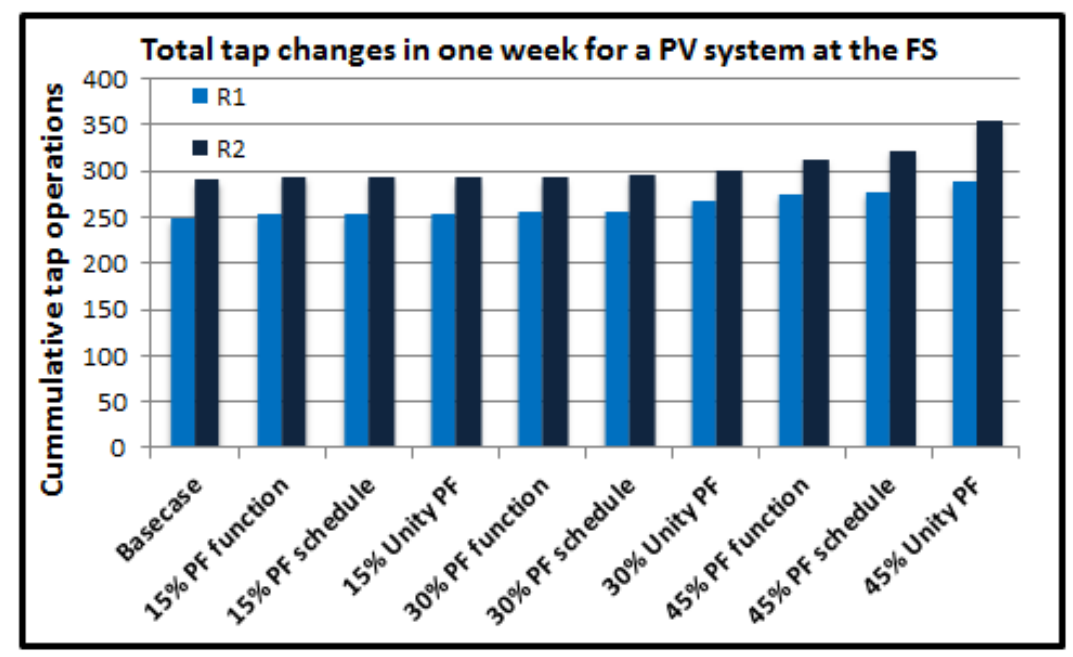

Figure 6.21: Total tap operations in the sample week for the basecase and PV deployed at FS with various PF control strategy

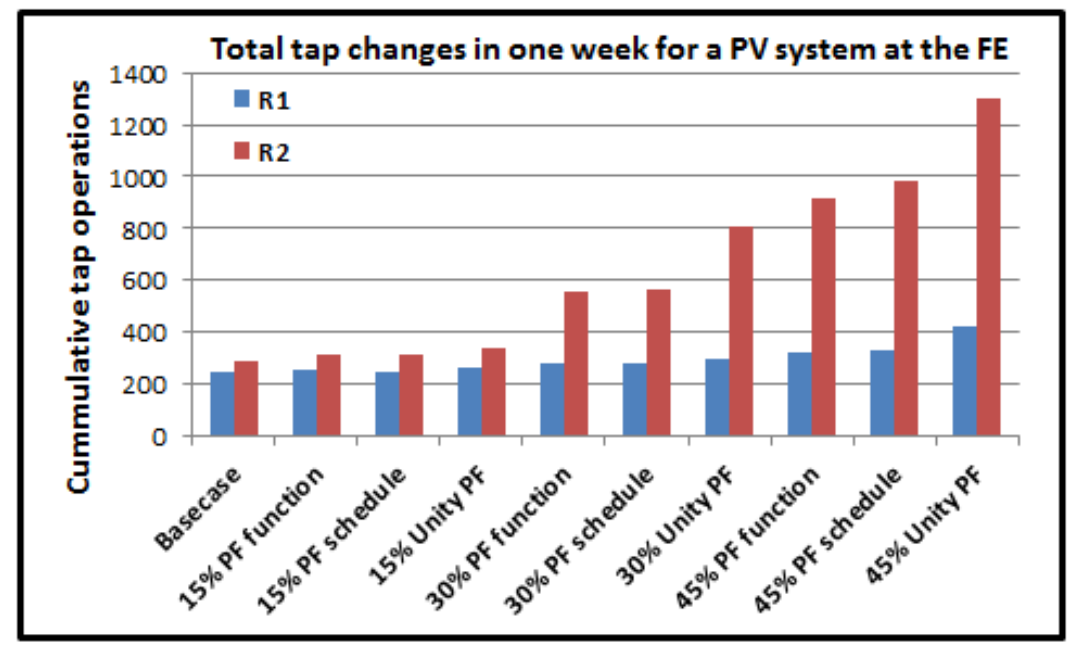

Figure 6.22: Total tap operations in the sample week for the basecase and PV deployed at FE with various PF control strategy

The unity PF control strategy resulted in more tap changes for all PV 
deployment scenarios at the feeder source and end. In particular, PV plant operating at unity PF with $45 \%$ penetration caused the most tap change operations. For the PV system close to the FS, the percentage increase in tap operation for $\mathrm{R} 1$ and $\mathrm{R} 2$ are $16 \%$ and $22 \%$ respectively. On the other hand, For the PV system close to the FE, the percentage increase for R1 and $\mathrm{R} 2$ are $72 \%$ and $350 \%$ respectively over the basecase. Therefore, the PV system at the FE caused more tap change operations in comparison with FS case.

This large difference is as a result of the mismatch between the timing of the solar irradiance variation and that of the demand profile for the residential loads deployed at the end of the feeder as shown in Figure 6.9. The fluctuations occurred during minimum load conditions and therefore, resulted in more tap change operations. Also, large PV plants installed at the feeder end known to be electrically weaker than other feeder sections, generate voltage swings which lead to increased LTC operations to maintain the feeder voltage within the ANSI C84.1 range.

\subsubsection{Impacts on $\mathrm{kW}$ and $\mathrm{kVAR}$ profiles}

It is pivotal for system operators to be able to visualize the impact of PV output variability on the feeder profile for $\mathrm{kW}$ and $\mathrm{kVAR}$ power flows on the lines especially in high penetration scenarios. This is because excessive $\mathrm{kW}$ and $\mathrm{kVAR}$ flows can use up feeder capacity leading to equipment overload, high voltage drop and power losses. Figure 6.23 shows the basecase plots for the 3-phase $\mathrm{kW}$ and kVAR profiles on the distribution lines.

\section{Feeder kW profiles}

As depicted by Figure 6.24, there is a significant reduction of $\mathrm{kW}$ power flow due to PV deployment at $45 \%$ penetration level close to FS and FE. These reductions have the ability to cause a peak load relief for the power delivery system, power loss reduction, increased capacity and reliability of 


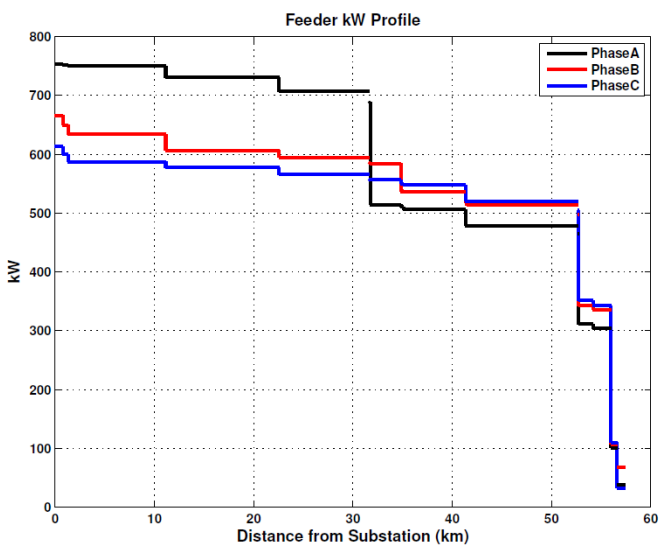

(a) Basecase $\mathrm{kW}$ power flow

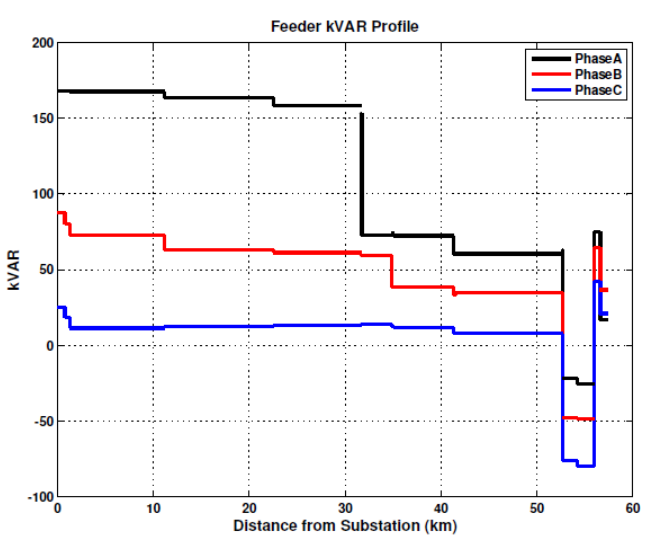

(b) Basecase kVAR power flow

Figure 6.23: Basecase $\mathrm{kW}$ and kVAR power flows on the feeder lines

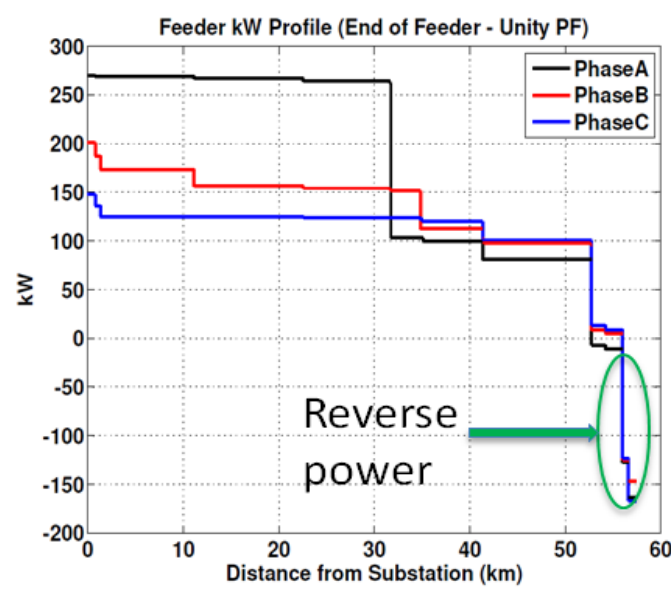

(a) $\mathrm{kW}$ power flow with PV at FE

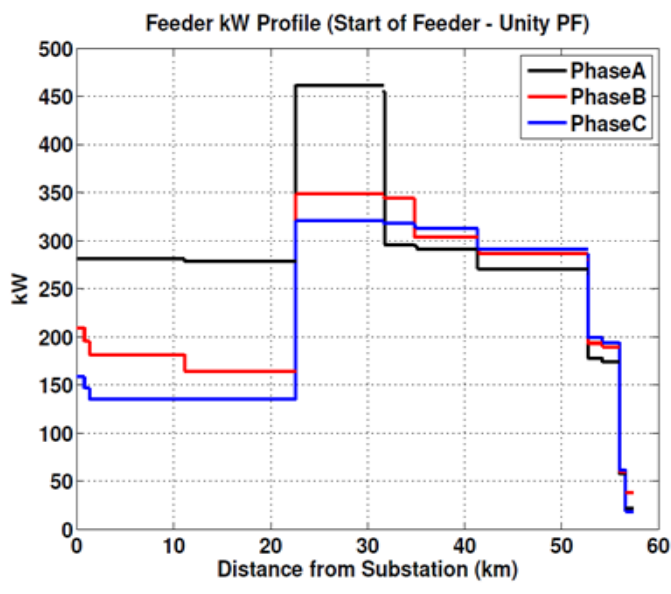

(b) $\mathrm{kW}$ power flow with PV at FS

Figure 6.24: $\mathrm{kW}$ power flows offset with PV systems 
the distribution network. However, at the feeder end close to the point of PV deployment, there is a reverse power flow which usually occurs during times of minimum load and high PV production. The obvious questions is, is this large enough to violate any system constraints such as voltage? The previous voltage analysis confirms that there is no possible violation with this amount of reverser power flow.

In addition, wires and fixed-ratio transformers have the capacity to handle bidirectional power flows before being overloaded. However, large reverse power flow can cause control and protection issues if device settings are not accurately configured [184].

For the PV deployed close to the substation, although active power reduced at the point of PV integration, it is higher at other feeder sections. This implies that this site close to the substation is a less optimal location than buses close to the feeder end.

\section{Feeder kvar profiles}

Figure 6.25 shows the impact of PV with different PF control strategies on the feeder $\mathrm{kVar}$ profile. Almost all the reactive power supply from the grid are used up in the case of unity PF control strategy. On the other hand, for PF schedule there is a reactive power supply on the black phase (within $30 \mathrm{~km}$ from substation) which is needed for voltage support.

Also, about $100 \mathrm{kVar}$ and $150 \mathrm{kVar}$ are being absorbed from the grid on the red and blue phases respectively for Unity PF while approximately $50 \mathrm{kVar}$ and $100 \mathrm{kVar}$ on the same phases for PF schedule. The figure for PF function is omitted because it is quite similar with PF schedule. Also, similar pattern of impact occurred close to the substation except that there is higher kvar power flow offset at the feeder end than source. 


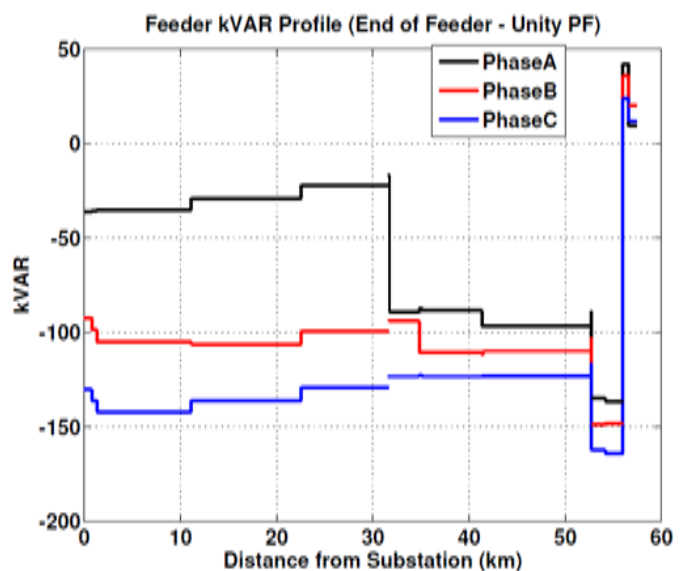

(a) kVar profile with unity PF

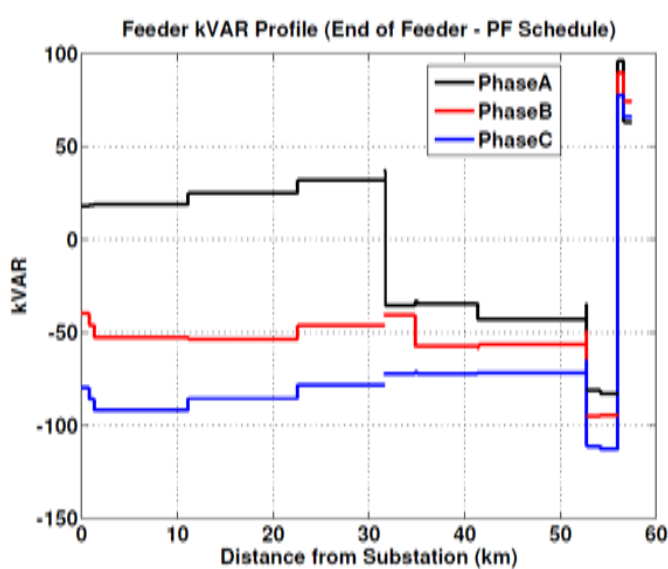

(b) kVar profile with PF schedule

Figure 6.25: Comparison of kVar power flows with different PF strategies

\subsection{Chapter summary}

In this chapter, impacts of three power factor $(\mathrm{PF})$ control schemes (PF schedule, PF function and fixed PF) on the power output of a centralized utility interactive PV plant deployed close to the feeder source and end using WVM at various penetration levels have been presented.

Since PV output determines its impacts, this research has analysed impacts of PV output variability on voltage profile, voltage regulator OLTC operations and power flows by taken advantage of the upscale from a single module and point irradiance sensor to the geographic smoothing in the WVM.

Results showed that, although variability increases with PV system deployed near the feeder source and end, it is higher at the feeder end than source. Also, unity PF strategy resulted in higher maximum and minimum feeder voltage with less headroom than other control strategies.

In addition, the PV plant close to the feeder end caused the most voltage profile enhancement than the one near the source. Furthermore, the PV plant at the rear caused the highest tap change operations than the one 
close to the source. The large difference is as a result of the mismatch between the timing of the solar irradiance variation and that of the demand profile for residential loads deployed close to the feeder end. Also, large PV plants installed at the feeder end known to be electrically weaker than other feeder sections, generate voltage swings which lead to increased LTC operations to maintain the feeder voltage within the ANSI C84.1 range.

\subsection{Next chapter}

The next chapter concludes this thesis with the major contributions and future works. 


\section{Chapter 7}

\section{Conclusions and future research}

\subsection{Conclusions}

The overall goal of this thesis is to conduct an interconnection impact analysis of solar PV systems at increasing penetration levels subject to the feeder constraints within the distribution network. This is carried out with a time series power flow analysis method to capture the time-varying nature of solar PV and load, with their interactions with the distribution network device operations.

In order to enable a graceful uptake of the PV technology into the evolving active distribution system, accurate modelling is pivotal for true impact assessment and the provision of smart grid mitigation alternatives. Therefore, this thesis analyses multiple PV systems scenarios resulting in a wide range of possible impacts to enable distribution system planners and operators to understand and characterize grid operation with the integration of PV systems.

The Solar PV technology is currently the most common DG integrated with the electric power system and therefore, has become a vital renewable energy resource for the evolving smart grid. The initial motivation behind this thesis arose from the investigation of the performance of a grid-tied PV system in a school setting that has a close matching peak demand with 
the solar generation, which helps to offset peak demand during the midday. Moreover, such utility interactive systems are monitored to evaluate the final energy yield, identify operational problems and increase their penetration into the existing electric power infrastructure.

The proliferation of spatially distributed single-phase grid-tied PV systems coupled with their high tendency to cause emergent behaviours calls for an impact assessment study such as the school one described earlier. An interconnection study for this PV system scenario with a typical school load profile and solar irradiance data at 5-minute resolution has been carried out on the IEEE 13-bus feeder. The performance of generator models, PQ and $P|V|$, for connecting PV-DG with the distribution feeder are compared with their respective computational costs for a converged power flow solution. Moreover, this thesis uses standard IEEE bus systems because validated and accurate basecase models are required for performing model-based interconnection studies.

A method capable of computing the incremental capacity additions, measuring risks and upgrade deferral provided by PV systems deployments is proposed in this thesis. This research proposes surrogate metrics, energy exceeding normal rating and unserved energy, for evaluating system reliability and capacity usage. These two measures can be a very useful visualization tool for distribution network planners and operators. The intrinsic strong correlation that exists between distribution network capacity and system reliability gives the inclusion of renewable DG and the evaluation of their additional load serving capacity a prime place in the emerging active distribution system planning. Sensitivity analysis is performed for optimal location of the PV system on the distribution network. This is critical due to the strong relationship that exists between power losses and system capacity. The optimal integration of PV systems is often near-optimal for network capacity relief or release issues as well. In addition, our analysis carried out shows that apart from optimal siting and sizing, maximizing the value of PV-DG depends on its ability to 
generate power at proper times and levels.

The recent rapid adoption of centralized PV plants over distributed units due to governmental interventions with the provision of cheap capital and competitive tenders now creates an urgent modelling need of such centralized PV plants with high penetration scenarios. This thesis models the impact of centralized PV variability on the electric grid using the WVM which considers the key factors that affect PV variability such as PV footprint, density and cloud movement over the entire PV plant. The upscaling advantage from a single module and point irradiance sensor to geographic smoothing over the entire PV footprint in WVM is used to simulate effects of a grid-connected large PV system on the distribution feeder. In addition, this thesis proposes the use of advanced inverter functionalities such as power factor function and schedule control strategies over the fixed power factor technique. This is because the former provides the flexibility required by utilities to adjust the PV output without assuming a particular amount of plant output each day.

Importantly, the PV interconnection scenarios presented in this thesis have been modelled with different time scales ranging from seconds to hours in order to accurately capture and represent various impacts. For instance, effects with short time constants such as the impact of PV output variability on the on-load tap changing operations has been modelled with a high frequency irradiance data in seconds time scale. However, hourly and sub-hourly operational impact analysis are used to provide a wide overview of the PV generation impact on system performance, for example plant energy production and additional load serving capacity provided by PV systems.

\section{Contributions}

The major contributions of this thesis are as follows:

1. This research shows how to evaluate the operational and reliability 
performance of a grid-connected PV system based on the IEC standards (61836 and 61724), IEA PVPS and industry guides. The performance analysis metrics in thesis allow cross-comparison between PV systems operating under different climatic conditions and identification of operational issues. Results showed the significant impact of temperature on the overall performance of the PV system. Periods with low temperatures experienced higher performance ratio values, reduced array captured and system losses, while the reverse is true for higher temperature seasons. These results have been have been peer-reviewed and published in [19]:

M. Emmanuel, D. Akinyele, and R. Rayudu, Techno-economic analysis of a $10 \mathrm{kWp}$ utility interactive photovoltaic system at Maungaraki school, Wellington, New Zealand, Elsevier Energy, vol. 120, no. Supplement C, pp. 573-583, 2017.

2. In this thesis, the impact of spatially distributed single-phase PV systems on the distribution network with a five-minute resolution data of load and solar generation profiles is presented. Further, this thesis compares the performance of DG models, constant PQ and $P|V|$, in terms of local impacts such as bus voltage profiles and unbalance, and system-wide impacts which include feeder equipment loading, power losses and computational costs for a converged power flow solution. This research proposes the use of $P|V|$ over PQ model for integrating PV-DG with distribution network because it minimizes both local and system-wide impacts with reduced computational cost. These results have been have been peer-reviewed and published in [20]:

M. Emmanuel and R. Rayudu, The impact of single-phase gridconnected distributed photovoltaic systems on the distribution network using P-Q and $P|V|$ models, Elsevier International Journal of Electrical Power \& Energy Systems, vol. 91, pp. 20-33, 2017. 
3. The conventional reliability indices (CAIDI, SAIFI, etc.) use average values, which makes it difficult to notice a change if a small number of consumers experience enhancement in reliability as a result of DG integration. This thesis proposes surrogate metrics, energy exceeding normal rating and unserved energy, for evaluating system reliability which can be used as a visualization tool for distribution network planners and operators. This research shows how these metrics can be used to compute PV-DG incremental capacity additions and measurement of the period of upgrade deferral of the distribution system. In addition, the evaluation of these metrics can be used to assess system capacity usage and as a planning tool to measure the effectiveness of the applied DG incremental solution. Also, since a single year analysis may not be sufficient to characterize the dynamic distribution network due to the inevitable load growth, this study presents multi-year analysis considering a yearly demand growth on both lightly and heavily loaded networks. The analysis shown in this thesis is very useful in situations where the rate of demand increase is uncertain and the expansion of the network is very difficult and expensive. These results have been have been peer-reviewed and published in [21], [22]:

M. Emmanuel, R. K. Rayudu, and I. Welch, Grid capacity released analysis and incremental addition computation for distribution system planning, Elsevier Electric Power Systems Research, vol. 152, pp. 105-121, 2017.

M. Emmanuel, R. Rayudu and I. Welch, "Grid incremental capacity evaluation with an optimally deployed photovoltaic system in distribution network," 2017 IEEE Power \& Energy Society General Meeting, Chicago, IL, USA, 2017, pp. 1-5.

4. This thesis models the impact of variability and locational value of centralized PV plants in a distribution system with a high resolu- 
tion solar irradiance data (1 s) and geographic smoothing using the WVM. Here, a duty cycle dispatch simulation is used to investigate the true impacts of solar variability at various penetration levels, which is an important step in incorporating such a variable resource in the EPS operations. Further, the WVM is used to estimate the output of a centralized PV plants operating with three power factor control strategies (power factor function, power factor schedule and fixed power factor) at various penetration levels. Analysis of three scenarios, namely, PV-DG close to the feeder source and end for centralized PV deployments on the IEEE-34 bus feeder are presented. Impacts on the feeder active and reactive power profiles, voltage regulator tap changing operations, maximum and minimum feeder voltages are investigated. Also, two types of customers (i.e., commercial and residential) with different time-varying load patterns for an entire year (8760 hours) are incorporated in the distribution test feeder. These results have been have been peer-reviewed and published in [23]:

M. Emmanuel, R. Rayudu, and I. Welch, Impacts of power factor control schemes in time series power flow analysis for centralized PV plant using wavelet variability model, vol. 13, no. 6, pp. 31853194, 2017.

\subsection{Future work}

There is an obvious need for an effective modelling methodology to robustly meet the active distribution network transition challenge. It is very important to develop smart distribution and DG models needed to assess the impacts of high penetration of DER technologies and also, provide smart grid mitigation alternatives to address the adverse effects caused by DER integration. Possible future research areas are as follows: 
- Modelling and comparing variability impacts of distributed and centralized PV systems using the WVM. This will involve geographic smoothing across the entire PV plant, the use of high resolution timeseries and quasi-static time-series models to accurately assess the impacts of PV integration. Although quasi-static time-series analysis involves complex data requirements such as load and PV data at one second time resolution. Obtaining a high-resolution load data in the order of seconds remains a major challenge for this type of analysis.

- Modelling of centralized and distributed PV advanced inverter functionalities such as power factor control techniques, power curtailment, reactive power control and Volt/var.

- Coordination of the operation of advanced PV inverter functions with each other and existing network equipment.

- Computation of the incremental capacity additions of centralized and distributed DER technologies using surrogate reliability metrics, energy exceeding normal and unserved energy, based on the power delivery elements loading constraints within the distribution network.

For grid reliable operations, it is important to maintain a diverse generation portfolio, however, identifying the best RE resources from both engineering and economic perspectives is pivotal in ensuring cost effectiveness, reliability improvement and increased resource diversity. The engineering screening should entail capacity and reliability analysis coupled with highlights on timing, severity and location of constraints on the power system. Depending on the technical analysis, the economic analysis should determine the baseline avoided cost and cost-effectiveness of the DER sources. The developed model can be used to provide a realistic projection of the economic effects of the deployment of various DER technologies scenarios. Further, uncertainty analysis can be helpful in pro- 
viding the required refinement for the entire analysis to give a robust recommendation of the DER choice ${ }^{1}$.

\footnotetext{
${ }^{1}$ (This is part of a commentary submitted by author, peer-reviewed and published in [185])
} 


\section{Bibliography}

[1] D. Lew and N. Miller, "Reaching new solar heights: integrating high penetrations of PV into the power system," IET Renewable Power Generation, vol. 11, no. 1, pp. 20-26, 2017.

[2] B. Singh and S. Kumar, "Grid integration of 3P4W solar PV system using M-LWDF-based control technique," IET Renewable Power Generation, vol. 11, no. 8, pp. 1174-1181, 2017.

[3] H. D. Tafti, A. I. Maswood, G. Konstantinou, J. Pou, K. Kandasamy, Z. Lim, and G. H. P. Ooi, “Low-voltage ride-thorough capability of photovoltaic grid-connected neutral-point-clamped inverters with active/reactive power injection," IET Renewable Power Generation, vol. 11, no. 8, pp. 1182-1190, 2017.

[4] F. Katiraei and J. Aguero, "Solar PV Integration Challenges," Power and Energy Magazine, IEEE, vol. 9, no. 3, pp. 62-71, 2011.

[5] M. J. Reno and K. Coogan, "Grid Integrated Distributed PV (GridPV), Sandia National Laboratories SAND2013-6733, 2013.," tech. rep.

[6] S. Kouro, J. I. Leon, D. Vinnikov, and L. G. Franquelo, "GridConnected Photovoltaic Systems: An Overview of Recent Research and Emerging PV Converter Technology," IEEE Industrial Electronics Magazine, vol. 9, no. 1, pp. 47-61, 2015. 
[7] “UTILITY OF THE FUTURE : An MIT Energy Initiative response to an industry in transition," tech. rep., MIT Energy Initiative, 2009.

[8] “International Energy Agency PVPS : Trends 2016 in photovoltaic applications," tech. rep., 2016.

[9] "Integrating Renewable Electricity on the Grid:A Report by the APS Panel on Public Affairs," tech. rep., American Physical Society (APS), 2010.

[10] “Utility-scale PV variability Workshop, Cedar Rapids, Iowa," tech. rep., 2009.

[11] J. Cochran, D. Paul, B. Speer, and M. Miller, “Grid integration and the carrying capacity of the U.S. grid to incorporate varaiable renewable energy," tech. rep., 2015.

[12] M. Lave, A. Ellis, and J. S. Stein, "Simulating Solar Power Plant Variability:A Review of Current Methods, Sandia National Laboratories SAND2013-4757, 2013.," tech. rep.

[13] M. J. Reno, A. Ellis, J. Quiroz, and S. Grijalva, "Modeling Distribution System Impacts of Solar Variability and Interconnection Location," in World Renewable Energy Forum, 2012.

[14] D. Lew and R. Piwko, "Western wind and solar integration study, National Renewable Energy Laboratory, NREL/SR-55047434, 2010.," tech. rep.

[15] R. C. Dugan, "Computing Incremental Capacity Provided By Distributed Resources For Distribution Planning," in Power Engineering Society General Meeting, 2007. IEEE, pp. 1-5, IEEE, 2007.

[16] S. Price, C. Baskette, B. Horii, D. Lloyd, P. Light, M. King, R. Dugan, and L. King, " Renewable DG Assessment: Alameda Power \& Telecom Case Study. California Energy Commission, PIER Renewable 
Energy Technologies, Energy and Environmental Economics, Inc. 2004, 500-01-042.," tech. rep., Jan. 2004.

[17] M. Mosadeghy, R. Yan, and T. K. Saha, "A Time-Dependent Approach to Evaluate Capacity Value of Wind and Solar PV Generation," IEEE Transactions on Sustainable Energy, vol. 7, pp. 129-138, Jan 2016.

[18] M. Lave, M. J. Reno, and R. J. Broderick, "Characterizing local highfrequency solar variability and its impact to distribution studies," Solar Energy, vol. 118, no. Supplement C, pp. 327-337, 2015.

[19] M. Emmanuel, D. Akinyele, and R. Rayudu, "Techno-economic analysis of a $10 \mathrm{kWp}$ utility interactive photovoltaic system at Maungaraki school, Wellington, New Zealand," Energy, vol. 120, no. Supplement C, pp. 573-583, 2017.

[20] M. Emmanuel and R. Rayudu, "The impact of single-phase gridconnected distributed photovoltaic systems on the distribution network using P-Q and P-V models," International Journal of Electrical Power \& Energy Systems, vol. 91, pp. 20-33, 2017.

[21] M. Emmanuel, R. K. Rayudu, and I. Welch, "Grid capacity released analysis and incremental addition computation for distribution system planning," Electric Power Systems Research, vol. 152, pp. 105-121, 2017.

[22] M. Emmanuel, R. Rayudu, and I. Welch, "Grid incremental capacity evaluation with an optimally deployed photovoltaic system in distribution network," in 2017 IEEE Power Energy Society General Meeting, pp. 1-5, July 2017.

[23] M. Emmanuel, R. Rayudu, and I. Welch, "Impacts of power factor control schemes in time series power flow analysis for centralized 
PV plant using wavelet variability model," vol. 13, no. 6, pp. 31853194, 2017.

[24] M. Emmanuel and R. Rayudu, "Evolution of dispatchable photovoltaic system integration with the electric power network for smart grid applications: A review ," Renewable and Sustainable Energy Reviews, vol. 67, pp. 207-224, 2017.

[25] F. Bizzarri, M. Bongiorno, A. Brambilla, G. Gruosso, and G. S. Gajani, "Model of Photovoltaic Power Plants for Performance Analysis and Production Forecast," IEEE Transactions on Sustainable Energy, vol. 4, pp. 278-285, April 2013.

[26] D. L. King, W. E. Boyson, and J. A. Kratochvill, "Photovoltaic Array Performance Model, Sandia National Laboratories SAND2004-3535, 2004.," tech. rep.

[27] D. L. King, W. E. Boyson, and J. A. Kratochvil, "Analysis of factors influencing the annual energy production of photovoltaic systems," in Conference Record of the Twenty-Ninth IEEE Photovoltaic Specialists Conference, 2002., pp. 1356-1361, May 2002.

[28] D. L. King, W. E. Boyson, and J. A. Kratochvill, "Evaluation of the National Solar Radiation Database (NSRDB): 1998-2015 , National Renewable Energy Laboratory, NREL/TP-5D00-67722, 2017.," tech. rep.

[29] "PV Performance Modelling Collaborative, An Industry and Sandia National Laboratory collaborative to Improve Photovoltaic Performance Modeling, 2017.[Online]. Available: https:/ /pvpmc.sandia.gov/modeling-steps /."

[30] “IEEE Application Guide for IEEE Std 1547TM, IEEE Standard for Interconnecting Distributed Resources with Electric Power Systems , 2008. 
[31] M. Emmanuel, R. Rayudu, and W. Seah, "Dispatchable distributed energy resources for seamless integration into the smart grid," IEEE Smart Grid Newsletter, 2016.

[32] R. Walling, R. Saint, R. Dugan, J. Burke, and L. Kojovic, "Summary of Distributed Resources Impact on Power Delivery Systems," Power Delivery, IEEE Transactions on, vol. 23, no. 3, pp. 1636-1644, 2008.

[33] M. H. Bollen and F. Hassan, Integration of distributed generation in the power system, vol. 80. John wiley \& sons, 2011.

[34] "C.J. Mozina. The impact of distributed generation. PAC World (Power, Automation and Control Magazine), 2008.," tech. rep.

[35] N. Patapoff Jr and D. Mattijetz, "Utility interconnection experience with an operating central station MW-sized photovoltaic plant," Power Apparatus and Systems, IEEE Transactions on, no. 8, pp. 20202024, 1985.

[36] W. Jewell and R. Ramakumar, "The effects of moving clouds on electric utilities with dispersed photovoltaic generation," Energy Conversion, IEEE Transactions on, no. 4, pp. 570-576, 1987.

[37] O. Ceylan, G. Liu, Y. Xu, and K. Tomsovic, "Distribution system voltage regulation by distributed energy resources," in North American Power Symposium (NAPS), 2014, pp. 1-5, IEEE, 2014.

[38] P. Basak, S. Chowdhury, S. H. nee Dey, and S. Chowdhury, "A literature review on integration of distributed energy resources in the perspective of control, protection and stability of microgrid," Renewable and Sustainable Energy Reviews, vol. 16, no. 8, pp. 5545-5556, 2012.

[39] R. Dugan, T. S. Key, and G. Ball, "Distributed resources standards," Industry Applications Magazine, IEEE, vol. 12, no. 1, pp. 27-34, 2006. 
[40] “IEEE Application Guide for IEEE Std 1547a-2014, IEEE Standard for Interconnecting Distributed Resources with Electric Power Systems, Amendment 1," 2008.

[41] D. Q. Hung, N. Mithulananthan, and K. Y. Lee, “Determining PV penetration for distribution systems with time-varying load models," Power Systems, IEEE Transactions on, vol. 29, no. 6, pp. 30483057, 2014.

[42] O. Wasynczuk, "Modeling and dynamic performance of a linecommutated photovoltaic inverter system," Energy Conversion, IEEE Transactions on, vol. 4, no. 3, pp. 337-343, 1989.

[43] O. Wasynczuk and N. Anwah, "Modeling and Dynamic Performance of a Self-Commutated Photovoltaic Inverter System," Power Engineering Review, IEEE, vol. 9, no. 9, pp. 33-34, 1989.

[44] L. Wang and Y.-H. Lin, "Dynamic stability analyses of a photovoltaic array connected to a large utility grid," in Power Engineering Society Winter Meeting, 2000. IEEE, vol. 1, pp. 476-480 vol.1, IEEE, 2000.

[45] O. Wasynczuk, D. Carroll, G. Gareis, P. Krause, C. Ong, and R. Schwartz, "Dynamic simulation of dispersed, grid-connected photovoltaic power systems: System studies," tech. rep., Purdue Univ., Lafayette, IN (USA). School of Electrical Engineering, 1985.

[46] R. S. Sugimura and J. M. Wood, "Utility Application of Photovoltaic Power Generation: A Survey of Recent Literature," Power Engineering Review, IEEE, vol. PER-7, no. 12, pp. 30-31, 1987.

[47] J. Arrillaga, Y. H. Liu, N. R. Watson, and N. J. Murray, Selfcommutating converters for high power applications. John Wiley \& Sons, 2010. 
[48] F. D. Bianchi, A. Egea-Alvarez, A. Junyent-Ferré, and O. GomisBellmunt, "Optimal control of voltage source converters under power system faults," Control Engineering Practice, vol. 20, no. 5, pp. 539-546, 2012.

[49] S. Jain and V. Agarwal, "A Single-Stage Grid Connected Inverter Topology for Solar PV Systems With Maximum Power Point Tracking," Power Electronics, IEEE Transactions on, vol. 22, no. 5, pp. 19281940, 2007.

[50] S. Yang Chen, "A cost-effective single-stage inverter with maximum power point tracking," Power Electronics, IEEE Transactions on, vol. 19, no. 5, pp. 1289-1294, 2004.

[51] M. Armstrong, D. Atkinson, C. Johnson, and T. Abeyasekera, "AutoCalibrating DC Link Current Sensing Technique for Transformerless, Grid Connected, H-Bridge Inverter Systems," Power Electronics, IEEE Transactions on, vol. 21, no. 5, pp. 1385-1393, 2006.

[52] S. Kjaer, J. Pedersen, and F. Blaabjerg, "A review of single-phase grid-connected inverters for photovoltaic modules," Industry Applications, IEEE Transactions on, vol. 41, no. 5, pp. 1292-1306, 2005.

[53] R. Comfort, A. Mansoor, and A. Sundaram, "Power quality impact of distributed generation: effect on steady state voltage regulation," in PQA 2001 North America Conference, Pittsburgh, Pennsylvania, 2001.

[54] M. M. Begovic, I. Kim, D. Novosel, J. R. Aguero, and A. Rohatgi, “Integration of Photovoltaic Distributed Generation in the Power Distribution Grid," in 2012 45th Hawaii International Conference on System Sciences, pp. 1977-1986, Jan 2012.

[55] M. Rylander, J. Smith, D. Lewis, and S. Steffel, "Voltage impacts from distributed photovoltaics on two distribution feeders," in Power and Energy Society General Meeting (PES), 2013 IEEE, pp. 1-5, IEEE, 2013. 
[56] F. Shahnia, R. Majumder, A. Ghosh, G. Ledwich, and F. Zare, "Sensitivity analysis of voltage imbalance in distribution networks with rooftop PVs," in Power and Energy Society General Meeting, 2010 IEEE, pp. 1-8, IEEE, 2010.

[57] Y. Liu, J. Bebic, B. Kroposki, J. De Bedout, and W. Ren, "Distribution system voltage performance analysis for high-penetration PV," in Energy 2030 Conference, 2008. ENERGY 2008. IEEE, pp. 1-8, IEEE, 2008.

[58] R. Tonkoski, D. Turcotte, and T. H. El-Fouly, "Impact of high PV penetration on voltage profiles in residential neighborhoods," Sustainable Energy, IEEE Transactions on, vol. 3, no. 3, pp. 518-527, 2012.

[59] S. Weckx, C. Gonzalez, and J. Driesen, "Reducing grid losses and voltage unbalance with PV inverters," in PES General Meeting-Conference $\mathcal{E}$ Exposition, 2014 IEEE, pp. 1-5, IEEE, 2014.

[60] M. Thomson and D. Infield, "Impact of widespread photovoltaics generation on distribution systems," Renewable Power Generation, IET, vol. 1, no. 1, pp. 33-40, 2007.

[61] J. Smith, R. Dugan, and W. Sunderman, "Distribution modeling and analysis of high penetration PV," in Power and Energy Society General Meeting, 2011 IEEE, pp. 1-7, IEEE, 2011.

[62] A. Hoke, R. Butler, J. Hambrick, and B. Kroposki, "Steady-State Analysis of Maximum Photovoltaic Penetration Levels on Typical Distribution Feeders," IEEE Transactions on Sustainable Energy, vol. 4, no. 2, pp. 350-357, 2013.

[63] V. M. Quezada, J. R. Abbad, and T. G. S. Roman, "Assessment of energy distribution losses for increasing penetration of distributed generation," IEEE TRANSACTIONS ON POWER SYSTEMS PWRS, vol. 21, no. 2, p. 533, 2006. 
[64] I. El-Samahy and E. El-Saadany, "The effect of DG on power quality in a deregulated environment," in IEEE Power Engineering Society General Meeting, 2005, pp. 2969-2976 Vol. 3, IEEE, 2005.

[65] H. L. Willis, "Analytical methods and rules of thumb for modeling DG-distribution interaction," in Power Engineering Society Summer Meeting, 2000. IEEE, vol. 3, pp. 1643-1644, IEEE, 2000.

[66] D. L. Garrett and S. M. Jeter, "A Photovoltaic Voltage Regulation Impact Investigation Technique: Part I-Model Development," IEEE Power Engineering Review, vol. 9, no. 3, pp. 29-30, 1989.

[67] E. C. Kern, E. M. Gulachenski, and G. A. Kern, "Cloud effects on distributed photovoltaic generation: slow transients at the Gardner, Massachusetts photovoltaic experiment," IEEE Transactions on Energy Conversion, vol. 4, no. 2, pp. 184-190, 1989.

[68] A. Hariri, M. O. Faruque, R. Soman, and R. Meeker, "Impacts and interactions of voltage regulators on distribution networks with high PV penetration," in North American Power Symposium (NAPS), 2015, pp. 1-6, IEEE, 2015.

[69] H. Ravindra, M. O. Faruque, K. Schoder, M. Steurer, P. Mclaren, and R. Meeker, "Dynamic interactions between distribution network voltage regulators for large and distributed PV plants," in PES T D 2012, pp. 1-8, May 2012.

[70] Y. P. Agalgaonkar, B. C. Pal, and R. A. Jabr, “Distribution Voltage Control Considering the Impact of PV Generation on Tap Changers and Autonomous Regulators," IEEE Transactions on Power Systems, vol. 29, pp. 182-192, Jan 2014.

[71] G. K. Ari and Y. Baghzouz, "Impact of high PV penetration on voltage regulation in electrical distribution systems," in 2011 Interna- 
tional Conference on Clean Electrical Power (ICCEP), pp. 744-748, June 2011.

[72] M. Emmanuel, R. Rayudu, and I. Welch, "Distributed Energy Resources Integration Standards for Smart Grid Applications," IEEE Smart Grid Newsletter, October, 2016.

[73] “IEEE 1547 and 2030 Standards for Distributed Energy Resources Interconnection and Interoperability $w$ ith the Electricity Grid, NREL/TP -5D00 -63157," tech. rep., National Renewable Energy Laboratory, 2014.

[74] U. Jahn, D. Mayer, M. Heidenreich, R. Dahl, S. Castello, L. Clavadetscher, A. Frölich, B. Grimmig, W. Nasse, K. Sakuta, et al., “International Energy Agency PVPS T13: Analytical monitoring of grid-connected photovoltaic systems," p. 5, Citeseer, 2000.

[75] B. Marion, J. Adelstein, K. Boyle, H. Hayden, B. Hammond, T. Fletcher, B. Canada, D. Narang, A. Kimber, L. Mitchell, G. Rich, and T. Townsend, "Performance parameters for grid-connected PV systems," in Photovoltaic Specialists Conference, 2005. Conference Record of the Thirty-first IEEE, pp. 1601-1606, IEEE, 2005.

[76] U. Jahn, D. Mayer, M. Heidenreich, R. Dahl, S. Castello, L. Clavadetscher, A. Frölich, B. Grimmig, W. Nasse, K. Sakuta, et al., "International Energy Agency PVPS Task 2: Analysis of the operational performance of the IEA Database PV systems," in 16th European Photovoltaic Solar Energy Conference and Exhibition, Glasgow, United Kingdom, p. 5, Citeseer, 2000.

[77] S. Pietruszko and M. Gradzki, "Performance of a grid connected small PV system in Poland," Applied energy, vol. 74, no. 1, pp. 177184, 2003. 
[78] J. D. Mondol, Y. Yohanis, M. Smyth, and B. Norton, "Long term performance analysis of a grid connected photovoltaic system in Northern Ireland," Energy Conversion and Management, vol. 47, no. 18, pp. 2925-2947, 2006.

[79] S. Chokmaviroj, R. Wattanapong, and Y. Suchart, "Performance of a 500kW P grid connected photovoltaic system at Mae Hong Son Province, Thailand," Renewable Energy, vol. 31, no. 1, pp. 19-28, 2006.

[80] E. Kymakis, S. Kalykakis, and T. M. Papazoglou, "Performance analysis of a grid connected photovoltaic park on the island of Crete," Energy Conversion and Management, vol. 50, no. 3, pp. 433-438, 2009.

[81] F. Cherfa, A. H. Arab, R. Oussaid, K. Abdeladim, and S. Bouchakour, "Performance Analysis of the Mini-grid Connected Photovoltaic System at Algiers," Energy Procedia, vol. 83, pp. 226-236, 2015.

[82] M. Adaramola, "Viability of grid-connected solar PV energy system in Jos, Nigeria," International Journal of Electrical Power and Energy Systems, vol. 61, pp. 64-69, 2014.

[83] M. Mondal and A. Sadrul Islam, "Potential and viability of gridconnected solar PV system in Bangladesh," Renewable Energy, vol. 36, pp. 1869-1874, 2011.

[84] M. El-Shimy, "Viability analysis of PV power plants in Egypt," Renewable Energy, vol. 34, pp. 2187-2196, 2009.

[85] M. Adaramola, "Techno-economic analysis of a $2.1 \mathrm{~kW}$ rooftop photovoltaic-grid-tied system based on actual performance," Energy Conversion and Management, vol. 101, pp. 85-93, 2015.

[86] "The Dynamis Project, http://www.dynamisproject.co.nz/."

[87] D. Akinyele, R. Rayudu, and N. Nair, "Global progress in photovoltaic technologies and the scenario of development of solar panel 
plant and module performance estimation- Application in Nigeria," Renewable and Sustainable Energy Reviews, vol. 48, pp. 112-139, 2015.

[88] S. Labed and E. Lorenzo, "The impact of solar radiation variability and data discrepancies on the design of PV systems," Renewable Energy, vol. 29, no. 7, pp. 1007-1022, 2004.

[89] "Enasolar Grid-tied Solar Inverter, http://www.enasolar.net/Products/EnaSolar-GT-Series-SolarInverter-v2-3f."

[90] “Solar Photovoltaic Energy Systems, IEC/TS 61836 (2007-12)."

[91] “PV System Performance Monitoring, IEC 61724.."

[92] "Design of grid connect PV systems," tech. rep., Palau Public Utilities Corporation.

[93] "IEEE Guide for Array and Battery Sizing in Stand-Alone Photovoltaic (PV) Systems," 2008.

[94] D. Akinyele, R. Rayudu, and R. H. Tan, "Comparative Study of Photovoltaic Technologies Based on Performance, Cost and Space Requirement: Strategy for Selection and Application," International Journal of Green Energy, 2015.

[95] D. Akinyele, R. Rayudu, and N. Nair, "Development of photovoltaic power plant for remote residential applications: The socio-technical and economic perspectives," Applied Energy, vol. 155, pp. 131-149, 2015.

[96] K. Padmavathi and S. A. Daniel, "Performance analysis of a 3MW p grid connected solar photovoltaic power plant in India," Energy for Sustainable Development, vol. 17, no. 6, pp. 615-625, 2013. 
[97] B. S. Kumar and K. Sudhakar, "Performance evaluation of $10 \mathrm{MW}$ grid connected solar photovoltaic power plant in India," Energy Reports, vol. 1, pp. 184-192, 2015.

[98] V. Sharma and S. Chandel, "Performance analysis of a $190 \mathrm{kWp}$ grid interactive solar photovoltaic power plant in India," Energy, vol. 55, pp. 476-485, 2013.

[99] J.-Y. Kim, G.-Y. Jeon, and W.-H. Hong, “The performance and economical analysis of grid-connected photovoltaic systems in Daegu, Korea," Applied energy, vol. 86, no. 2, pp. 265-272, 2009.

[100] "Power System Operation and Augmentation Planning with PV Integration," tech. rep., International Energy Agency (2014), http://www.iea-pvps.org/index.php?id=322.

[101] "Grid-connected solar PV systems-no battery, Design guidelines for accreditted installers, Clean Energy Council (2013)."

[102] D. Akinyele and R. Rayudu, "Community-based hybrid electricity supply system: A practical and comparative approach," Applied Energy, vol. 171, pp. 608-628, 2016.

[103] "Renewable Power Generation Costs in 2014 (IRENA), http://www.irena.org/documentdownloads /publications/ irena_re_power_costs_2014_report.pdf/."

[104] T. Palm and A. Qayum, Private and public investment analysis, SouthWestern Pub. Co. 1985.

[105] W. Short, D. J. Packey, and T. Holt, A manual for the economic evaluation of energy efficiency and renewable energy technologies. University Press of the Pacific, 2005. 
[106] A. Miller, A. Wood, M. Hwang, S. Lemon, and E. G. Read, “Economics of photovoltaic solar power and uptake in New Zealand," 2015.

[107] R. C. Dorf, The Technology Management Handbook. Heidelberg: Springer, 1999.

[108] "National Institute of Water and Atmospheric Research, http:/ / www.niwa.co.nz/our-services/online-services/solarview."

[109] L. Ayompe, A. Duffy, S. McCormack, and M. Conlon, "Measured performance of a $1.72 \mathrm{~kW}$ rooftop grid connected photovoltaic system in Ireland," Energy conversion and management, vol. 52, no. 2, pp. 816-825, 2011.

[110] D. Okello, E. van Dyk, and F. Vorster, "Analysis of measured and simulated performance data of a $3.2 \mathrm{kWp}$ grid-connected PV system in Port Elizabeth, South Africa," Energy Conversion and Management, vol. 100, pp. 10-15, 2015.

[111] S. Edalati, M. Ameri, and M. Iranmanesh, "Comparative performance investigation of mono-and poly-crystalline silicon photovoltaic modules for use in grid-connected photovoltaic systems in dry climates," Applied Energy, vol. 160, pp. 255-265, 2015.

[112] "Photovoltaic (PV) Systems-Characteristics of the Utility Interface, IEC 61727:2004, Dec. 2004.."

[113] "Standard for Inverters, Converters, Controllers and Interconnection System Equipment for Use With Distributed Energy Resources, UL 1741.."

[114] D. Parmar and L. Yao, "Impact of Unbalanced Penetration of Single Phase Grid Connected Photovoltaic Generators on Distribution 
Network," in Universities' Power Engineering Conference (UPEC), Proceedings of 2011 46th International, pp. 1-8, VDE, 2011.

[115] M. A. Awadallah, B. Venkatesh, and B. N. Singh, "Impact of Solar Panels on Power Quality of Distribution Networks and Transformers," Canadian Journal of Electrical and Computer Engineering, vol. 38, no. 1, pp. 45-51, 2015.

[116] A. El-Naggar and I. Erlich, "Control approach of three-phase grid connected PV inverters for voltage unbalance mitigation in lowvoltage distribution grids," IET Renewable Power Generation, vol. 10, no. 10, pp. 1577-1586, 2016.

[117] F. Gao and M. R. Iravani, "A Control Strategy for a Distributed Generation Unit in Grid-Connected and Autonomous Modes of Operation," IEEE Transactions on Power Delivery, vol. 23, no. 2, pp. 850-859, 2008.

[118] L. A. Gallego, E. Carreno, and A. Padilha-Feltrin, "Distributed generation modelling for unbalanced three-phase power flow calculations in smart grids," in Transmission and Distribution Conference and Exposition: Latin America (TEamp;D-LA), 2010 IEEE/PES, pp. 323328, IEEE, 2010.

[119] M. Z. Kamh and M. R. Iravani, "A Sequence Frame-Based Distributed Slack Bus Model for Energy Management of Active Distribution Networks.," IEEE Trans. Smart Grid, vol. 3, no. 2, pp. 828-836, 2012.

[120] C. Schauder and H. Mehta, "Vector analysis and control of advanced static VAr compensators," IEE Proceedings C - Generation, Transmission and Distribution, vol. 140, no. 4, pp. 299-306, 1993. 
[121] M. S., Srinivas, "Distribution load flows: a brief review," in Power Engineering Society Winter Meeting, 2000. IEEE, vol. 2, pp. 942-945 vol.2, IEEE, 2000.

[122] W. H. Kersting, Distribution system modeling and analysis. CRC press, 2012.

[123] C. S. Cheng and D. Shirmohammadi, "A three-phase power flow method for real-time distribution system analysis," IEEE Transactions on Power Systems, vol. 10, no. 2, pp. 671-679, 1995.

[124] M. Z. Kamh and R. Iravani, "Unbalanced Model and Power-Flow Analysis of Microgrids and Active Distribution Systems," IEEE Transactions on Power Delivery, vol. 25, no. 4, pp. 2851-2858, 2010.

[125] S. Bolognani and S. Zampieri, "On the Existence and Linear Approximation of the Power Flow Solution in Power Distribution Networks," IEEE Transactions on Power Systems, vol. 31, no. 1, pp. 163172, 2016.

[126] S. Khushalani, J. M. Solanki, and N. N. Schulz, "Development of Three-Phase Unbalanced Power Flow Using PV and PQ Models for Distributed Generation and Study of the Impact of DG Models," IEEE Transactions on Power Systems, vol. 22, no. 3, pp. 1019-1025, 2007.

[127] S. H. Hashemi, M. H. Ashouian, H. Pirpiran, and R. Karami, "Impact of distributed generation on unbalanced distribution networks," in Electricity Distribution (CIRED 2013), 22nd International Conference and Exhibition on, pp. 1-4, IET, 2013.

[128] S. Moghaddas-Tafreshi and E. Mashhour, "Distributed generation modeling for power flow studies and a three-phase unbalanced 
power flow solution for radial distribution systems considering distributed generation," Electric Power Systems Research, vol. 79, no. 4, pp. 680-686, 2009.

[129] K. Maya and E. Jasmin, "A Three Phase Power Flow Algorithm for Distribution Network Incorporating the Impact of Distributed Generation Models," Procedia Technology, vol. 21, pp. 326-331, 2015.

[130] "Transpower System Operator (2016), http://www.systemoperator.co.nz/system-operations/publisheddata/load-graphs."

[131] D. Q. Hung, N. Mithulananthan, and R. Bansal, "Analytical strategies for renewable distributed generation integration considering energy loss minimization," Applied Energy, vol. 105, pp. 75-85, 2013.

[132] "Bibliography on load models for power flow and dynamic performance simulation," IEEE Transactions on Power Systems, vol. 10, no. 1, pp. 523-538, 1995.

[133] Z. Salameh, B. Borowy, and A. Amin, "Photovoltaic module-site matching based on the capacity factors," Energy Conversion, IEEE Transactions on, vol. 10, no. 2, pp. 326-332, 1995.

[134] M. Khallat and S. Rahman, "A probabilistic approach to photovoltaic generator performance prediction," IEEE Transactions on Energy Conversion, no. 3, pp. 34-40, 1986.

[135] D. K. Khatod, V. Pant, and J. Sharma, "Evolutionary programming based optimal placement of renewable distributed generators," IEEE Transactions on power systems, vol. 28, no. 2, pp. 683-695, 2013.

[136] Y. M. Atwa, E. F. El-Saadany, M. M. A. Salama, and R. Seethapathy, “Optimal Renewable Resources Mix for Distribution System En- 
ergy Loss Minimization," IEEE Transactions on Power Systems, vol. 25, no. 1, pp. 360-370, 2010.

[137] S. Tong, Slack bus modeling for distributed generation and its impacts on distribution system analysis, operation and planning. PhD thesis, Drexel University, 2006.

[138] F. Ugranlı and E. Karatepe, "Multiple-distributed generation planning under load uncertainty and different penetration levels," International Journal of Electrical Power E Energy Systems, vol. 46, pp. 132144, 2013.

[139] D. Q. Hung and M. Nadarajah, "DG allocation in primary distribution systems considering loss reduction," 2011.

[140] "IEEE Standard Test Procedure for Polyphase Induction Motors and Generators ," 2008.

[141] W. H. Kersting, "Radial distribution test feeders," in Power Engineering Society Winter Meeting, 2001. IEEE, vol. 2, pp. 908-912, IEEE, 2001.

[142] M. Ropp, J. Newmiller, C. Whitaker, and B. Norris, "Review of potential problems and utility concerns arising from high penetration levels of photovoltaics in distribution systems," in 2008 33rd IEEE Photovolatic Specialists Conference, 2008.

[143] H. D. Mathur, N. C. Hien, N. Mithulananthan, D. Joshi, and R. C. Bansal, “Distributed generation: a power system perspective," 2011.

[144] J. Federico, V. Gonzalez, and C. Lyra, "Learning classifiers shape reactive power to decrease losses in power distribution networks," in IEEE Power Engineering Society General Meeting, 2005, pp. 557-562 Vol. 1, IEEE, 2005.

[145] S. Price, M. Baskette, B. Horii, P. Light, R. Dugan, and L. King, “ Renewable DG Assessment: City of Palo Alto Utilities Case Study. 
California Energy Commission, PIER Renewable Energy Technologies, Energy and Environmental Economics, Inc. 2004, 500-01-042.," tech. rep., Jan. 2005.

[146] J. Bebic, " Power system planning: emerging practises suitable for evaluating the impact of high-penetration photovoltaics, http://www.nrel.gov/docs/fy08osti/42297.pdf," tech. rep., Feb. 2008.

[147] M. A. Abdullah, K. M. Muttaqi, A. P. Agalgaonkar, and D. Sutanto, "Capacity deferral credit evaluation of renewable distributed generation," in 2013 IEEE Industry Applications Society Annual Meeting, pp. 1-8, IEEE, 2013.

[148] R. Dugan and M. Waclawiak, "Using energy as a measure of risk in distribution planning," in International conference on electricity distribution, 2007. CIRED, pp. 1-4, CIRED, 2007.

[149] M. A. Abdullah, K. M. Muttaqi, A. P. Agalgaonkar, and D. Sutanto, "A Noniterative Method to Estimate Load Carrying Capability of Generating Units in a Renewable Energy Rich Power Grid," IEEE Transactions on Sustainable Energy, vol. 5, no. 3, pp. 854-865, 2014.

[150] S. H. Madaeni, R. Sioshansi, and P. Denholm, "Estimating the Capacity Value of Concentrating Solar Power Plants: A Case Study of the Southwestern United States," IEEE Transactions on Power Systems, vol. 27, no. 2, pp. 1116-1124, 2012.

[151] R. Perez, M. Taylor, T. Hoff, and J. P. Ross, "Reaching Consensus in the Definition of Photovoltaics Capacity Credit in the USA: A Practical Application of Satellite-Derived Solar Resource Data," IEEE Journal of Selected Topics in Applied Earth Observations and Remote Sensing, vol. 1, no. 1, pp. 28-33, 2008. 
[152] J. Haslett and M. Diesendorf, "The capacity credit of wind power: A theoretical analysis," Solar Energy, vol. 26, no. 5, pp. 391-401, 1981.

[153] S. H. Madaeni, R. Sioshansi, and P. Denholm, "Comparing Capacity Value Estimation Techniques for Photovoltaic Solar Power," IEEE Journal of Photovoltaics, vol. 3, no. 1, pp. 407-415, 2013.

[154] L. L. Garver, "Effective Load Carrying Capability of Generating Units," IEEE Transactions on Power Apparatus and Systems, vol. PAS85, pp. 910-919, Aug 1966.

[155] K. Dragoon and V. Dvortsov, "Z-method for power system resource adequacy applications," IEEE Transactions on Power Systems, vol. 21, no. 2, pp. 982-988, 2006.

[156] C. D'Annunzio and S. Santoso, "Analysis of a wind farm's capacity value using a non-iterative method," in Power Engineering Society General Meeting-Conversion and Delivery of Electrical Energy in the 21st Century, 2008. IEEE, pp. 1-8, IEEE, 2008.

[157] B. Hasche, A. Keane, and M. O'Malley, “Capacity Value of Wind Power, Calculation, and Data Requirements: the Irish Power System Case," IEEE Transactions on Power Systems, vol. 26, no. 1, pp. 420-430, 2011.

[158] S. H. Madaeni, R. Sioshansi, and P. Denholm, "Comparison of capacity value methods for photovoltaics in the western United States, NREL," tech. rep., 2012.

[159] S. H. Madaeni, R. Sioshansi, and P. Denholm, "The capacity value of solar generation in the Western United States," in 2012 IEEE Power and Energy Society General Meeting, pp. 1-8, IEEE, 2012.

[160] A. Keane, M. Milligan, C. J. Dent, B. Hasche, C. D'Annunzio, K. Dragoon, H. Holttinen, N. Samaan, L. Soder, and M. O’Malley, “Ca- 
pacity Value of Wind Power," IEEE Transactions on Power Systems, vol. 26, no. 2, pp. 564-572, 2011.

[161] C. J. Dent, A. Keane, and J. W. Bialek, "Simplified methods for renewable generation capacity credit calculation: A critical review," in IEEE PES General Meeting, pp. 1-8, IEEE, 2010.

[162] "Electric Power Research Institute (EPRI), OpenDSSWiki."

[163] R. Dugan and S. Price, "Including distributed resources in distribution planning," in CIRED 2005 - 18th International Conference and Exhibition on Electricity Distribution, pp. 1-5, IET, 2005.

[164] D. Q. Hung, N. Mithulananthan, and R. C. Bansal, "Analytical Expressions for DG Allocation in Primary Distribution Networks," IEEE Transactions on Energy Conversion, vol. 25, no. 3, pp. 814-820, 2010.

[165] D. Popović, J. Greatbanks, M. Begović, and A. Pregelj, “Placement of distributed generators and reclosers for distribution network security and reliability," International Journal of Electrical Power \& Energy Systems, vol. 27, no. 5, pp. 398-408, 2005.

[166] J. Candelo and H. Hernández, "Location and Size of Distributed Generation to Reduce Power Losses using a Bat-inspired Algorithm," in VII Simposio Internacional sobre Calidad de la Energía Eléctrica, 2013.

[167] "A Sensitivity Analysis Toolkit for the Simplification of MV Distribution Network Voltage Management," Smart Grid, IEEE Transactions on, vol. 5, no. 2, pp. 559-568, 2014.

[168] K. Christakou, J.-Y. LeBoudec, M. Paolone, and D.-C. Tomozei, “Efficient Computation of Sensitivity Coefficients of Node Voltages 
and Line Currents in Unbalanced Radial Electrical Distribution Networks," IEEE Transactions on Smart Grid, vol. 4, no. 2, pp. 741-750, 2013.

[169] M. Brenna, E. D. Berardinis, L. D. Carpini, F. Foiadelli, P. Paulon, P. Petroni, G. Sapienza, G. Scrosati, and D. Zaninelli, "Automatic Distributed Voltage Control Algorithm in Smart Grids Applications," IEEE Transactions on Smart Grid, vol. 4, no. 2, pp. 877-885, 2013.

[170] “National Renewable Energy Laboratory's System Advisor Model (SAM)."

[171] "Distribution System Analysis Subcommittee, IEEE 34 node test feeder," tech. rep.

[172] "International Energy Agency PVPS : 2016 Snapshot of Global Photovoltaic Markets," tech. rep., 2016.

[173] M. Lave, J. Kleissl, and J. S. Stein, "A Wavelet-Based Variability Model (WVM) for Solar PV Power Plants," IEEE Transactions on Sustainable Energy, vol. 4, pp. 501-509, April 2013.

[174] M. Lave and J. Kleissl, “Cloud speed impact on solar variability scaling - Application to the wavelet variability model," Solar Energy, vol. 91, no. Supplement C, pp. 11-21, 2013.

[175] A. R. Dyreson, E. R. Morgan, S. H. Monger, and T. L. Acker, "Modeling solar irradiance smoothing for large PV power plants using a 45-sensor network and the Wavelet Variability Model," Solar Energy, vol. 110, no. Supplement C, pp. 482-495, 2014.

[176] M. Lave and J. Kleissl, "Testing a wavelet-based variability model (WVM) for solar PV power plants," in 2012 IEEE Power and Energy Society General Meeting, pp. 1-6, July 2012. 
[177] C. Paravalos, E. Koutroulis, V. Samoladas, T. Kerekes, D. Sera, and R. Teodorescu, "Optimal Design of Photovoltaic Systems Using High Time-Resolution Meteorological Data.," IEEE Trans. Industrial Informatics, vol. 10, no. 4, pp. 2270-2279, 2014.

[178] G. Mokryani, A. Majumdar, and B. C. Pal, "Probabilistic method for the operation of three-phase unbalanced active distribution networks," IET Renewable Power Generation, vol. 10, no. 7, pp. 944-954, 2016.

[179] "Trends 2016 in Photovoltaic Applications, Survey Report of Selected IEA Countries between 1992 and 2015," tech. rep., International Energy Agency.

[180] "Relative merits of distributed vs. central photovoltaic (PV) generation, 2004, ," tech. rep., Navigant Consulting.

[181] J. Kleissl, M. Lave, M. Jamaly, and J. L. Bosch, "Aggregate solar variability," in 2012 IEEE Power and Energy Society General Meeting, pp. 13, July 2012.

[182] R. Broderick, J. Quiroz, M. Reno, A. Ellis, J. Smith, and R. Dugan, “Time Series Power Flow Analysis for Distribution Connected PV Generation," tech. rep., 2013.

[183] "Google. Google Maps API Family.."

[184] B. Palmintier, R. Broderick, B. Mather, M. Coddington, K. Baker, F. Ding, M. Reno, M. Lave, and A. Bharatkumar, "On the Path to SunShot: Emerging Issues and Challenges in Integrating Solar with the Distribution System," tech. rep., 2016.

[185] M. Emmanuel, R. Rayudu, and I. Welch, “Techno-Economic Screening Analysis for Variable Renewable Energy Sources- A ForwardLooking Alternative for a Seamless Baseload Generation Retirement," IEEE Smart Grid Newsletter, January, 2018. 